Portland State University

PDXScholar

Spring 6-2-2014

\title{
The Effects of Organizational Justice and Exercise on the Relationship between Job Stressors and Employee Health
}

Ana Cristina B. Costa

Portland State University

Follow this and additional works at: https://pdxscholar.library.pdx.edu/open_access_etds

Part of the Psychology Commons

Let us know how access to this document benefits you.

\section{Recommended Citation}

Costa, Ana Cristina B., "The Effects of Organizational Justice and Exercise on the Relationship between Job Stressors and Employee Health" (2014). Dissertations and Theses. Paper 1853.

https://doi.org/10.15760/etd.1852

This Dissertation is brought to you for free and open access. It has been accepted for inclusion in Dissertations and Theses by an authorized administrator of PDXScholar. Please contact us if we can make this document more accessible: pdxscholar@pdx.edu. 
The Effects of Organizational Justice and Exercise on the Relationship

between Job Stressors and Employee Health

by

Ana Cristina B. Costa

A dissertation submitted in partial fulfillment of the requirements for the degree of

Doctor of Philosophy

in

Applied Psychology

Dissertation Committee:

Donald Truxillo, Chair

Leslie Hammer

Charlotte Fritz

Matthew Carlson

Portland State University

2014 


\begin{abstract}
Recent decades have seen an explosion of research centered on understanding the influential impact that job stressors have on employees' subjective well-being, and now more recently, on objective assessments of physical health. Utilizing baseline data from a larger study funded by the National Institute for Occupational Safety and Health (NIOSH), I conducted a field study on blue-collar employees from two organizations in the construction industry, with the goal of exploring the impact of job stressors on job satisfaction (subjective well-being) and body mass index (objective health), as well as the influence of organizational justice as a moderator and exercise as a mediator in those relationships.

In support of previous research, results show that job stressors (job demands, low skill discretion, and low decision authority) all had significant direct effects on job satisfaction in the expected directions, signifying that the fewer demands and more control one has in their work role, the more satisfied one is. Results also indicate that distributive and procedural justice have significant main effects on job satisfaction, illustrating that higher perceptions of justice are related to higher levels of job satisfaction.

With respect to objective health, the data provides empirical support for the relationship between job control (skill discretion and decision authority) and BMI; however, job demands did not have a significant main effect on BMI. More importantly, the rarely studied relationship between organizational justice and BMI was investigated (Robbins et al., 2012), with results indicating that procedural and
\end{abstract}


distributive justice are important influences on one’s BMI level.

Post hoc analyses revealed that distributive and procedural justice are two relevant mediators to consider in the job stressor-job satisfaction relationship, illustrating the importance of considering employees' fairness perceptions with regards to their satisfaction levels. Moreover, exercise was found to be a significant moderator to the relationship between job demands and BMI, as well as the relationship between distributive justice and job satisfaction, shedding light on physical activity within the work and health contexts as a factor that interacts with employees' perceptions of justice and their workload demands to impact their psychological and physical health. Considering the cross-sectional nature of these data, all mediation and moderation results should be interpreted with caution.

With empirical support found for the direct association between job stressors and organizational justice and the outcomes of job satisfaction and BMI, this study has significant implications for researchers and practitioners alike to further expand upon these findings and implement them into organizational practice in support of the Total Worker Health initiative, which aims to promote employee safety and health (Schill \& Chosewood, 2013). Results suggest a healthy workforce is the result of the combination of employers transforming the work environment into a more just, transparent and trustworthy place to work, starting with the dynamics between supervisors and their employees, in conjunction with targeted interventions on employees' modifiable behaviors, such as engaging in physical activity and healthier eating habits. 


\section{Dedication}

To my parents, whose life of hard work, sacrifice, and dedication allowed their children to succeed in their endeavors.

You gave us everything, and you keep on giving.

This is for you.

To our health -

He who has health, has hope; and he who has hope, has everything.

Arabian Proverb 
Table of Contents

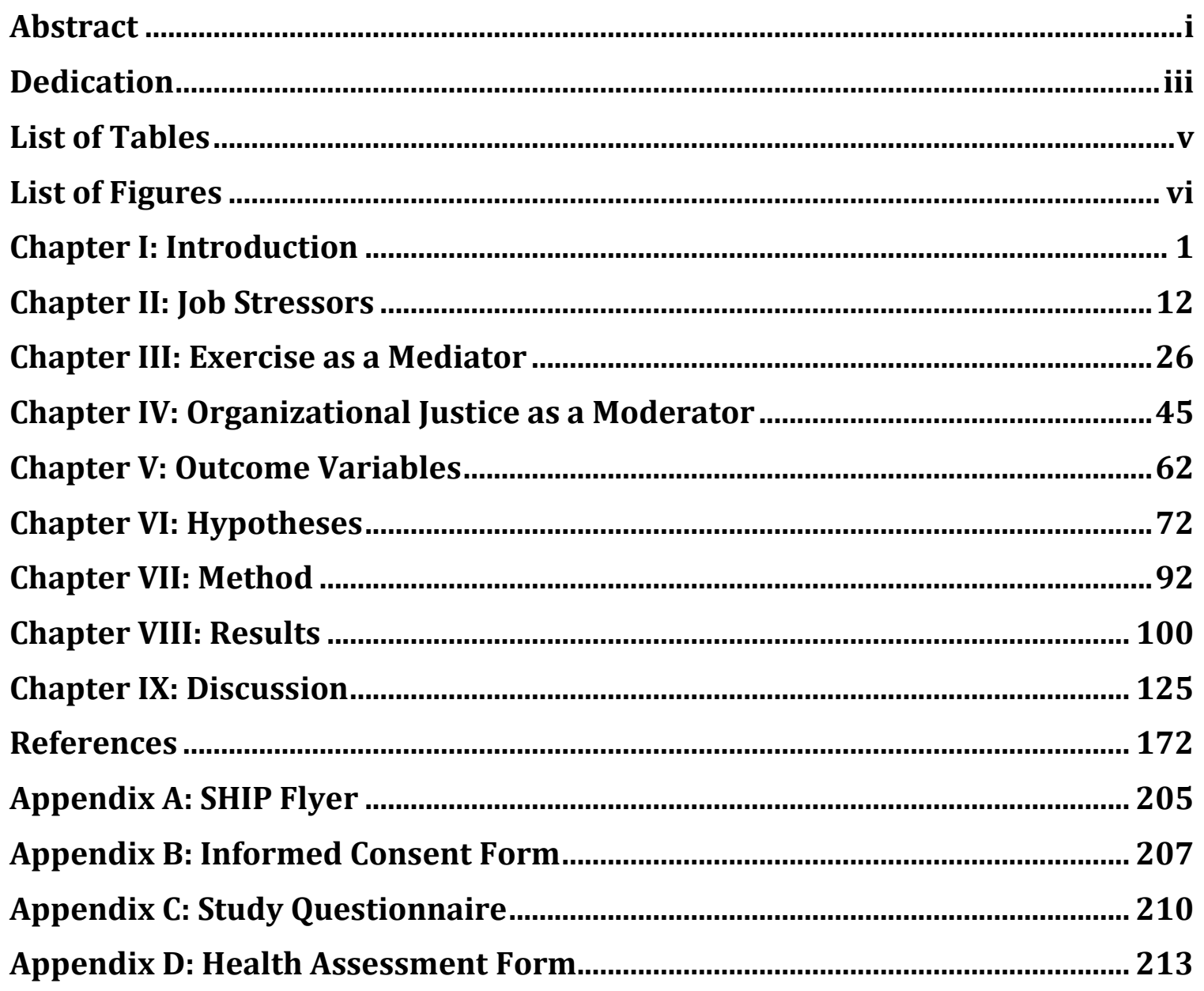




\section{List of Tables}

Table 1. Summary of Study Hypotheses and Hypothesis Results 147

Table 2. Means, Standard Deviations, Intercorrelations, and Alpha Reliability Statistics for Study Variables

Table 3. Main Effects of Job Stressors on Job Satisfaction (H1a-H1c) ...........................151

Table 4. Main Effects of Organizational Justice on Job Satisfaction (H2a-H2b) ........152

Table 5. Interaction of Distributive Justice and Job Demands on Job Satisfaction (H3a)

Table 6. Interaction of Procedural Justice and Job Demands on Job Satisfaction (H3b)

Table 7. Interaction of Distributive Justice and Skill Discretion on Job Satisfaction (H4a) 155

Table 8. Interaction of Procedural Justice and Skill Discretion on Job Satisfaction (H4b)

Table 9. Interaction of Distributive Justice and Decision Authority on Job Satisfaction (H5a) 157

Table 10. Interaction of Procedural Justice and Decision Authority on Job Satisfaction (H5b) 158

Table 11. Main Effects of Job Stressors on BMI (H6a-H6c) 159

Table 12. Main Effect of Organizational Justice on BMI (H7a-H7b). 160

Table 13. Interaction of Distributive Justice and Job Demands on BMI (H8a) ..........161

Table 14. Interaction of Procedural Justice and Job Demands on BMI (H8b) ............162

Table 15. Interaction of Distributive Justice and Skill Discretion on BMI (H9a) ......163

Table 16. Interaction of Procedural Justice and Skill Discretion on BMI (H9b)........164

Table 17. Interaction of Distributive Justice and Decision Authority on BMI (H10a) 165

Table 18. Interaction of Procedural Justice and Decision Authority on BMI (H10b)

Table 19. Exercise as a Mediator on the Relationship between Job Stressors and BMI (H11a-H11c) 167

Table 20. Exercise as a Mediator on the Relationship between Job Stressors and Job Satisfaction (H11d-H11f). 168 


\section{List of Figures}

Figure 1. Model of hypothesized relationships and study results .............................169

Figure 2. Interaction of Exercise and Job Demands on BMI .........................................170

Figure 3. Interaction of Exercise and Distributive Justice on Job Satisfaction...........171 


\section{Chapter I: Introduction}

Health, defined by the World Health Organization (1948) as "a complete state of physical, mental, and social well-being, and not merely the absence of disease or infirmity," has long been a topic of interest to practitioners and researchers. With health care costs on the rise at nearly three times the rate of inflation, and a projection that they will account for nearly $20 \%$ of the U.S. gross national product by 2015 (Borger, Smith, Truffer, Keehan, Siska, Poisal, \& Clemens, 2006), organizations have started to focus a lot more of their attention on what it actually means to have a 'healthy workforce' (Robbins, Ford, \& Tetrick, 2012; Smith, Cowan, Heffler, \& Catlin, 2006).

Incidentally, the direct medical costs only account for a portion of the expenditures faced by organizations when employees are not "healthy." The real expenses to the organization are, in fact, the ones associated with productivity loss, absenteeism, medical leave, turnover, and the use of compensation and disability programs, which may account for more than 50 percent of the total costs associated with poor employee health (Goetzel, Guindon, Turshen, \& Ozminkowski, 2001). Interestingly, research has also shown that the aforementioned list is often reported to be the consequences caused by work stress (Communications Workers of America, 2011).

In fact, according to the American Psychological Association (APA; 2010), $69 \%$ of individuals reported work as a significant source of stress, with more than half of the respondents expressing that they were less productive as a result of 
stress experienced during work, and 52\% reporting that they have considered looking for a new job, declining a promotion or leaving a job based on workplace stress. Sapolsky (1998) perfectly highlighted the phenomenon of how prolonged exposure to stress in general impacts our lives in the following quote:

"Stress-related disease emerges, predominantly, out of the fact that we so often activate a physiological system that has evolved for responding to acute physical emergencies, but we turn it on for months on end, worrying about mortgages, relationships, and promotions." (p. 7)

Although freedom from illness and the absence of negative conditions is still the norm in defining health in the still-prevalent medical model, today's focus is changing toward a more positive view of health (Seligman \& Csikszentmihalyi, 2000). One of the ways in which Industrial/Organizational (I/O) and Occupational Health Psychology (OHP) researchers are attempting to do so is by examining the relationship between work stressors and individuals' levels of subjective well-being, and more recently, objective assessments of their health to promote a healthier and more desirable work environment (Ganster \& Rosen, 2013; Robbins et al., 2012).

The blossoming work stress literature over the past 30 years has shown us that occupational stressors are negatively related to employee attitudes and psychological well-being (Ganster \& Rosen, 2013; Robbins et al., 2012), and in particular job satisfaction (Cahill, 1992; Dunham, Pierce, \& Castaneda, 1987; Elovainio, Kivimaki, Steen, \& Kalliomaki-Levanto, 2000; Elovainio, Kivimaki, \& Helkama, 2001; Karasek, 1979; Karasek \& Theorell, 1990; Nelson \& Simmons, 2003; Parkes \& Sparkes, 1998; Semmer, 2003; Tepper, 2001; Theorell \& Karasek, 1996). Job satisfaction, in turn, is perhaps one of the most widely studied concepts in I/O 
Psychology (Hulin \& Judge, 2003; Judge, Parker, Colbert, Heller, \& Ilies, 2002) in that it's been shown to impact organizational outcomes of interest such as focal performance, contextual performance, lateness, absence, and turnover intentions, and, ultimately, turnover (Harrison, Newman, \& Roth, 2006).

Kenny and colleagues (2000) posit that current trends in employment conditions may be eroding levels of job satisfaction and directly damaging the physical and mental health of employees (Kenny, Carlson, McGuigan, \& Sheppard, 2000). A recent meta-analysis examining the relationship between job satisfaction and both physical and mental health found that job satisfaction levels are an important factor impacting the health of workers (Faragher, Cass, \& Cooper, 2005). The researchers postulate that organizations should identify and eradicate work practices that cause the most job dissatisfaction along with the implementation of stress management policies as part of any exercise aimed at improving employee health. They also add that because of the important role that one's job plays in their life, occupational health clinicians should work with employees diagnosed as having psychological problems to critically evaluate their work and help them to explore ways of gaining greater satisfaction (Faragher et al., 2005).

In addition to job dissatisfaction, preliminary occupational stress studies demonstrate that high strain jobs (high job demands and low job control) are related to objective health outcomes, such as body mass index (BMI). BMI has become more prominent in health research as a worrisome health outcome as it's been shown that overweight or obese individuals are at higher risk for a host of health problems. In particular, the more body fat that individuals have, the more 
likely they are to also develop high blood pressure, Type 2 diabetes, gallstones, sleep apnea, reproductive problems, and certain cancers (CDC, 2012; Nyberg, Heikkila, \& Fransson, et al., 2012).

Although BMI may be a potential barometer for more serious health concerns in the long run, it has scarcely been investigated as an outcome of interest in the work stress literature in and of itself (Bosma, Marmot, Hemingway, Nicholson, Brunner, \& Stansfeld, 1997; Bosma, Stansfeld, \& Marmot, 1998; Karasek, 1990; Karasek \& Theorell, 1990; Kivimaki, Head, Ferrie, Shipley, Brunner, Vahtera, \& Marmot, 2006; Kivimaki, Vahtera, Thomson, Griffiths, Cox, \& Pentti, 1997; Nyberg et al., 2012; Robbins et al., 2012; Schnall, Landsbergis, \& Baker, 1994). Additionally, the extent to which job stressors, such as job demands and job control, and BMI are connected remains unclear (Bosma et al., 1997; 1998), in part, due to small sample sizes or the fact that something else may be at play in the job stressor-BMI relationship, leading researchers to interpret the inconsistent findings as an absence of an association (Kivimaki et al., 2006).

Recent studies, however, have started to point to certain health behaviors, such as exercise, as a possible mechanism via which job stressors are associated with BMI change (Berset, Semmer, Elfering, Jacobshagen, \& Meir, 2011; Nyberg et al., 2012). Not only would this newfound understanding increase our awareness of the progression of diseases related to body weight, but it would also help with the development of targeted and preventative interventions on modifiable behaviors (Berset et al., 2011). This is extremely important to consider as Nyberg and colleagues (2012) noted in their study on over 160,000 individuals that 
interventions aimed at reducing job stressors alone are not effective in combating obesity at a population level. The public policy implication of this finding is suggestive of the fact that simply counseling and educating individuals to manage and lower their stress, to eat healthier food options, and to be more physically active is not enough to create a permanent and positive change. The biggest impact to individuals at risk would be the implementation of nationwide employment policies and/or state mandated laws making specific and targeted changes to alter toxic or stagnant work environments (CDC, 2013).

In addition to this emphasis on potential linking mechanisms between job stressors and employee well-being and objective health, researchers are also seeking to identify factors that may moderate or buffer those relationships (Robbins et al., 2012). Cropanzano and colleagues (2005) have suggested that mitigating stressful situations rooting from injustice at work is one of the viable ways for management to address employee's health concerns, as a result of the amount of time that employees spend in direct contact with their supervisors and their dependence on supervisors for information, tools and materials, and rewards.

Not surprisingly, organizational justice has been noted as a possible resource against the long-term harmful impact of workplace stressors (Elovainio et al., 2001; Robbins et al., 2012). In light of the social and interpersonal nature of justice (Lind \& Tyler, 1988), it is believed that employees who perceive their organizational leaders to be fair can ultimately trust that the organizational decisions made are reached in a fair and transparent manner, thereby reducing their feelings of mistrust, stress, and uncertainty (Robbins et al., 2012). 
Thus, in an effort to expand upon our existing knowledge of the relationship between job stressors and job satisfaction/BMI, this study investigates exercise as a partial mediator, or as a partial linking mechanism for the often mixed results found between job stressors and BMI (that is, the relationship is not be expected to be reduced to zero, but rather just simply reduced, after controlling the relationship between exercise and job satisfaction/BMI). Also important is the investigation of resource-inducing safeguards against work stressors. Accordingly, this study investigates the moderating effects of organizational justice on those same relationships between job stressors and job satisfaction/BMI in hopes of further solidifying the association between job stressors and employee health outcomes. I anticipate that high levels of organizational justice would strengthen the relationship between work stressors and job satisfaction, while low justice would further deteriorate it. Similarly, I propose that high levels of justice will help to offset the effects of high job demands and low control on BMI, while low levels of justice would further exacerbate the negative effects. A full model of the hypothesized relationships can be seen in Figure 1 .

\section{Contributions of this Dissertation to Research and Practice}

This study not only responds to the timely calling for work-stress research on employee well-being and health outcomes, but it also addresses some of the often noted gaps and limitations in the I/O and OHP literature.

The first research design contribution is the fact that this is a field study conducted with blue-collar employees (employees and supervisors) in the construction industry, a group that is typically under studied in I/O. Study 
participants were asked to report on their relationships with their coworkers and supervisors, as well as on their perceptions of justice and support received by their organization rather than responding to hypothetical employment scenarios, which often lack the emotion and the realism of an actual 'stressful' or 'unjust' experience. Participants completed surveys and health assessments onsite at the beginning of their workday.

Whereas most work-stress studies rely on self-reported measures of health (i.e., sickness absences, smoking, alcohol consumption, and physical activity levels), this study employed an objective assessment of health whereby several objective health measures were captured. Accordingly, a second research design contribution of this study is that participants underwent a physical health assessment whereby their height and weight were measured to calculate their BMI rather than merely relying on employees' self-report of their height, weight, and anthropometric health (Elovainio et al., 2000, 2001; Elovainio, Kivimaki, Vahtera, Keltikangas-Jarvinen, \& Virtanen, 2003; Howard \& Cordes, 2010; Robbins et al., 2012; Taris, Kalimo, \& Schaufeli, 2002). The mixed-source (from both supervisors and employees), mixedmethod design (self-report survey and health assessment) employed in this study is helpful in offsetting the potential limitations of common method bias that typically occurs with exclusively survey-based research (Podsakoff, Mackenzie, Jeong-Yeon, \& Podsakoff, 2003) and further adds to the methodological contributions of this study.

The work stress literature primarily investigates affective outcomes (i.e., anxiety, depression, emotional exhaustion) or acute stress-related health complaints (i.e., headaches, fatigue, gastrointestinal problems). Not only does this study 
corroborate previous findings on the established relationship between job stressors and job satisfaction (an affective outcome), but it also contributes to the field by investigating the relationship between three types of job stressors (job demands, low skill discretion, and low decision authority) and their impact on exercise (a health behavior) and, subsequently, one's BMI (an objective health outcome). Accordingly, a third research contribution is the measurement of the objective health outcome of BMI. The majority of health studies don't investigate BMI as an outcome of interest; however, I make the argument, that BMI is an equally important outcome to examine because it is largely preventable and an overt precursor to the more distal silent killers, such as hypertension, Type 2 diabetes, CHD, and certain cancers, that may take years to develop (Kivimaki et al., 2006). Establishing a connection between job stressors and BMI is pertinent not only for corroborating previous findings (Nyberg et al., 2012), but also as an important step in the future of health studies as research has shown that 32 to 35 percent of all deaths in the United States attributed to CHD, cancer, and diabetes could be prevented if all members of the population were physically active (Powell \& Blair, 1994). Targeted explorations of the relationships between job stressors, exercise and BMI would allow for pertinent workplace changes to occur (i.e., increasing job control, designating a time during the work day to be physically active) long before a more serious health outcome develops.

The fourth, and perhaps, most important contribution of this study is the investigation of the still scarce relationship between organizational justice and objective physical health (Robbins et al., 2012), and in particular BMI (Nyberg et al., 
2012; Nyberg, Fransson, Heikkila, et al., 2013). Whereas most health studies that investigate justice tend to treat it as one overall construct, I investigated two dimensions of justice (procedural and distributive) and their distinct and unique relationships with BMI. Additionally, in light of the surge of interest and accumulating substantiation on the list of buffers in the job stressor-health relationship, this study also examined the interactive relationships between job stressors and organizational justice and their impact on employees' reported levels of job satisfaction and BMI - all relationships that have not been studied in this particular combination before.

Job satisfaction and BMI are two important outcomes in the work stress literature that need to gain more traction if they are to be taken seriously by employers and organizational management with their eye on healthcare premiums and bottom line figures (Robbins et al., 2012; Simons \& Roberson, 2003). As such, this study falls seamlessly in line with the overall goals of the NIOSH Total Worker Health program, which advocates for health protection and health promotion by targeting risk factors for preventable chronic health conditions in the workplace, identifying more efficient uses of resources, and gaining a deeper understanding of the underlying connections between the health, safety and performance of workers to the overall health of the organization (Hammer \& Sauter, 2013). Overview of the Dissertation

The dissertation will proceed as follows. In Chapter Two, I explore the job stress literature, providing a brief description of job demands and job control, and a discussion of the Job Demands-Control Model (Karasek, 1979) as the theoretical 
mechanism for the relationship between job stressors and the outcomes of interest in this study - job satisfaction and BMI. In Chapter Three, I provide a brief overview of the benefits of engaging physical activity and discuss the Ego Depletion Model (Baumeister, Blatslavsky, Muraven, and Tice, 1998) as an explanation for how the health behavior of exercise may be a viable and logical pathway via which job stressors are related to job satisfaction and BMI.

In Chapter Four, I describe the two widely accepted dimensions of organizational justice dimensions known as distributive and procedural justice. Additionally, I provide evidence of the relationship between distributive and procedural justice and job satisfaction, and suggest that similar relationships also exist between the two dimensions and BMI. The Effort-Reward Imbalance Theory (ERI; Siegrist, 1996) is proposed as the theoretical explanation for distributive justice as a moderator in the relationship between job stressors and BMI, and the Relational Model of Authority (Tyler \& Lind, 1992) as the theoretical explanation for procedural justice as a moderator in the relationship between job stressors and job satisfaction.

In Chapter Five, I provide a definition of each outcome of interest in this study (job satisfaction and BMI), as well as a synopsis of their known antecedents, outcomes, control variables, and measurement based on empirical evidence in the literature. In Chapter Six, I state predictions regarding the interaction between job demands and control and organizational justice and their impact on job satisfaction and BMI, while also investigating the unique role that health behaviors (exercise) 
may have in partially mediating the relationship between job stressors and organizational justice on BMI and job satisfaction.

Chapter Seven describes the overall study design, measures used, participant recruitment procedures, and strategies for analyzing the hypotheses. Chapter Eight discusses the results of each of the analyses conducted in this study. Finally, Chapter Nine provides a general discussion of the results and their implications for both research and practice in the I/O and OHP fields, potential limitations of this study, and suggestions for future research. 


\section{Chapter II: Job Stressors}

The purpose of this chapter is to describe existing research on job stressors and their associated relationships with well-being and health. First, I will provide a definition of occupational stress, stressors, and strain, and briefly describe the various types of stressors that may bring about job strain. Next, I will provide empirical evidence on the relationship between job stressors and the outcomes of job satisfaction and BMI through the lens of the Job Demands-Control Model (JD-C; Karasek, 1979). Then I will discuss how the mixed empirical evidence on the relationship between job stressors and job satisfaction and BMI may be suggestive of the fact that the relationships may, in fact, be mediated through a health behavior, such as exercise, which will be discussed in more detail in Chapter Three. Definitions of Stress, Stressors, and Strain

The most widely used definition of stress by Hans Selye (1950) states that stress is a nonspecific arousal reaction in response to all situations that require mobilization of energy. Further, the definition implies that stress is a neutral concept, and thus not negative in itself, although it can become negative during certain conditions. According to Frone (2003), stressors are sources of stress in the workplace, and are conditions and events that can be grouped into distinct categories. The different categories include physical stressors (i.e., adverse working conditions, noise, dirt, pollution, heat, toxic substances), task-related job stressors (i.e., high time pressure, work overload, high work complexity, monotonous work, disruptions), role stressors (i.e., role ambiguity and role conflict), social stressors (i.e., 
interpersonal conflicts with supervisors and coworkers, sexual harassment, bullying, dealing with difficult customers), work-schedule related stressors (i.e., night and shift work), career-related stressors (i.e., job insecurity and poor career opportunities), traumatic events (i.e., disasters, accidents, war, death), and stressful change processes (i.e., organizational mergers, downsizing, implementation of new technologies).

Jex and Britt (2008) noted that stress is the process whereby prolonged exposure to stressors leads to job strain. Job strain, in turn, has been described as a "stress reaction" to stressors, and in particular, the cognitive, affective, and physical reactions to stress that occur when individuals are deprived of resources, as is the case during prolonged exposure to stressors (de Lange, Taris, Kompier, Houtman \& Bongers, 2003).

Taking it one step further, Ganster and Rosen (2013) define work stress as the process by which workplace psychological experiences and demands (stressors) produce both short-term (strains) and long-term changes in one's mental and physical health. Job strain, specifically, is the result of the joint effects of high job demands and low job control (Karasek, 1979; Karasek \& Theorell, 1990; Theorell \& Karasek, 1996). Research shows that high strain jobs contribute to lowered employee productivity and a greater risk of health-related problems. More specifically, they are related to subjective well-being symptoms, such as depression, anxiety and job dissatisfaction, as well as objective well-being outcomes, such as increased number of sick days absent from work and cardiovascular disease (Bosma et al., 1997; Nelson \& Simmons, 2003; Theorell \& Karasek, 1996). 
Job Demands-Control Model

Karasek's (1979) Job Demands-Control (JD-C) model (also referred to as the job strain model) is a situation-centered model that puts explicit focus on task characteristics of the workplace. It was initially created to explain patterns of depression, exhaustion, and job dissatisfaction, but was later expanded to include cardiovascular disease, poor health functioning, and sickness absenteeism (Kuper \& Marmot, 2003). The JD-C postulates that job strain results from the interaction or joint effects between (1) "psychological job demands" and (2) "job control", with the most stress or psychological strain resulting from jobs that have high demands and low control (Frone, 2003; Karasek \& Theorell, 1990). Its central tenet is that concurrent high job demands and low decision latitude (control) can have an adverse impact on one's subjective well-being and physical health (van der Doef \& Maes, 1999).

Karasek (1979) defined psychological job demands (or the workload demands placed on an individual) as psychological stressors present in the work environment, such as high time pressure, high working pace, and difficult and mentally exacting work. In that spirit, Fox, Dwyer and Ganster (1993) stressed that job demands are not necessarily physical demands, giving the example that a fast, hectic work pace could lead to an individual experiencing anxiety about maintaining that pace and the potential consequences of not completing the work.

Job control, on the other hand, refers to one's ability to control work activities and/or one's authority over decisions, and is primarily measured by assessing one's level of decision latitude (Karasek, 1979). The term “job decision 
latitude" consists of two components: decision authority and skill discretion. Having good influence over what should be done and how it should be done is synonymous with high authority over decisions. It is also referred to as task control (Karasek \& Theorell, 1990). And having the possibility to influence the development of an employee's competence, or if employees can decide how their knowledge is used and developed, is referred to as knowledge control or high intellectual discretion (Fox et al., 1993; Karasek \& Theorell, 1990; van der Doef \& Maes, 1999).

Karasek (1979) combined the two dimensions of job demands (high and low) and job control (high and low) in a two-by-two matrix of job types to illustrate the effects of the various combinations of work demands and control on individuals' health and well-being levels. Different combinations of job demands and job control levels result in four varying work situations: relaxed, passive, active, and strain. Relaxed work situations (or low strain) are characterized by low demands and high control, and are considered ideal in terms of reducing strain. Passive situations consist of low demands and low control, while active situations are the exact opposite (high demands and high control). High strain situations are work situations in which demands are high and control is low, which result in psychological stress reactions, such as job strain and negative health effects.

The JD-C model makes two chief predictions, the strain and buffer hypotheses. The strain hypothesis essentially states that the strongest adverse strain reactions (e.g., poor subjective health and well-being) will occur when job demands are high and workers' levels of control are low. Thus, the strain hypothesis posits that individuals working in high-strain jobs experience the lowest levels of well- 
being (van der Doef \& Maes, 1999). However, “active jobs," whereby both job demands and control are high, result in increased work motivation, learning and growth, and are expected to produce little harm to individuals. The buffer hypothesis states that job control can moderate the negative effects of job demands on well-being, and explicitly predicts an interactive effect of work demands and job control, in which job control moderates the effects of job demands on the well-being outcomes (van der Doef \& Maes, 1999). The Main Effects of Job Demands and Job Control

Research has generally supported the main effects of the JD-C model and typically failed to find an interaction effect. That is, that high job demands typically result in higher levels of stress, and that high levels of control are generally associated with lower stress levels (Sonnentag \& Frese, 2003). De Lange and colleagues (2003) reviewed 45 longitudinal studies and found that, based on five evaluation criteria, only 19 of the studies were deemed "high quality." Of the 19 high quality studies reviewed, $63 \%$ reported significant main effects of job demands in predicting physical and psychological indicators of strain (usually in conjunction with main effects of control). Additionally, they found that even high quality studies only provide modest support for the interactional effects of demands and control, with only eight supporting an interaction effect between job demands and control on stress outcomes (de Jonge, Bosma, Peter, \& Siegrist, 2000; de Lange et al., 2003). Although the main effects have largely been supported, different studies tout the relative importance of job demands versus job control as the chief predictor to consider in health studies and job redesign efforts (Kuper \& Marmot, 2003). 
The JD-C model provides the conceptual framework for Karasek's Job Content Questionnaire, initially developed to measure work-related social and psychological factors that contribute to cardiovascular disorders (Karasek, 1985; Karasek, Schwartz, \& Pieper, 1983). More recently it has been shown to predict health, motivational, and productivity outcomes (Bosma et al., 1997; de Jonge \& Kompier, 1997; Hemingway \& Marmot, 1999; Schnall et al., 1994; van der Doef \& Maes, 1998), and essentially backs up the hypothesis that job demands are a key predictor to consider when measuring subjective and objective health outcomes.

Sauter, Hurrell, and Cooper (1989) further supported this outlook by noting that control latitude is difficult to measure and that fundamental questions remain concerning the conceptualization and operationalization of the control construct. In support of that sentiment, Bosma and colleagues (1998) found that high demands predicted poor health functioning in women, but not men. Two gastrointestinal studies indicate that the likelihood of having gastrointestinal symptoms increases in both men and women when self-reported job decision latitude is low, but particularly so if psychological demands are reported to be high at work (Nyren, 1995; Westerberg \& Theorell, 1997). Kuper and Marmot (2003) found that high demands were related to future occurrence of all coronary heart disease (CHD), especially fatal CHD and non-fatal myocardial infarction (MI) in both men and women, whereas low decision latitude was predictive only of all CHD in men. The researchers also noted that the association was strengthened after adjustments for employment grade and SES variables, but that including traditional coronary risk factors in the model had little effect. Their concluding thoughts were simply that 
people with high job demands, and to a lesser extent, low control, are at an increased risk for developing CHD. These results are largely consistent with recent findings from the Whitehall II study, where high demands turned out to be a more important predictor of CHD events than was previously imagined (Kuper \& Marmot, 2003).

Conversely, Theorell (2003) posits that there is a consistent and strong relationship between loss of control and onset of illness. Bosma and colleagues (1997) showed that state employees in England who had lost a significant amount of decision latitude at work in a two-year interval had nearly double the risk of developing new CHD during follow-up, even after controlling for accepted biological risk factors such as smoking habits, serum lipids, and blood pressure. Early results from the Whitehall II study found that low decision latitude (self-report or independent assessment) predicted self-reported CHD and higher rates of shortand long-term sickness absence (Kuper \& Marmot, 2003). Another prospective casecontrol study on matched samples (men and women between the ages of 45 and 65 who had suffered a first MI and a matched group without CHD) found that men between the ages of 45 and 54 that had experienced significantly lowered levels of decision latitude in a 10-year span were twice as likely to be in the MI group (Theorell, Tsutsumi, Hallquist, Reuterwall, Hogstedt, Fredlund, Emlund, \& Johnson, 1998). With regards to the long-term effects of exposure to low decision latitude, Johnson and colleagues (1996) found evidence for the cumulative protective effect of high decision latitude up to a period of 15 to 20 years, with no additional cumulative effect after 20 years. Thus, these studies point to the fact that high 
decision latitude can be protective, while a lack of decision latitude is related to the risk of developing cardiovascular disease and gastrointestinal disorders, and it may be related to musculoskeletal disorders (Theorell, 2003).

In sum, in support of the main effects of both job demands and job control, Taris (2006, pp. 100) asserted, “...there is no question that the demand-control model is correct in identifying demands and control as major predictors of work stress and ill-health, this does not seem to apply to the idea that demands and control interact in affecting these outcomes."

The Relationship Between Job Stressors and Job Satisfaction

Stress research has consistently documented the deleterious effects of negative stressors on various indicators of subjective well-being, with job satisfaction as a chief organizational outcome of interest (Bhagat, McQuaid, Lindholm, \& Segovis, 1985; Karasek, 1979). Moreover, empirical evidence on the impact of stressors on immediate affective responses has consistently been upheld and supported since Karasek proposed and tested his JD-C model (1979), with good evidence for the main effects of job stressors, and in particular job demands, on job satisfaction (Beehr \& Glazer, 2005; Bellavia \& Frone, 2005; de Lange et al., 2003; Karasek, 1998; Karasek \& Theorell, 1990; Moyle, 1998; Quick, Quick, Nelson, \& Hurrell, 1997; Schnall et al., 1994; Theorell \& Karasek, 1996; van der Doef \& Maes, 1998). In fact, in a review of 63 empirical studies addressing the JD-C model and psychological health, van der Doef and Maes (1999) found considerable support for the strain hypothesis suggestive that working in high strain jobs is associated with lower job satisfaction. 
The notion that people in high stress jobs typically experience elevated levels of job dissatisfaction has also been discovered in both men and women, as well as in young and old individuals, even when controlling for job sector, demographic characteristics and organizational position (de Jonge et al., 2000; Theorell, 2003). Thus, in hopes of corroborating past research findings on the basis of the JD-C model, this study will also investigate if high job demands and low job control give rise to lowered levels of job satisfaction.

\section{Unclear Connection Between Job Stressors and BMI}

Kivimaki and colleagues (2006) have noted that despite the common understanding that job stressors and high BMI are both risk factors for CHD and other serious health problems, the extent to which they are connected remains unclear, leading the majority of researchers to interpret the inconsistent findings as an indication of the absence of an association. While some studies have found an association between high job demands, low control, and increased BMI (Hellerstedt \& Jeffery, 1997; Wamala, Wolk, \& Orth-Gomer, 1997; Niedhammer, Goldberg, Leclerc, David, Bugel, \& Landre, 1998; Lallukka, Laaksonen, Martikainen, SarlioLahteenkorva, \& Lahelma, 2005), other studies have reported no associations (Brisson, Larocque, Moisan, Vezina, \& Dagenais, 2000; Reed, LaCroix, Karasek, Miller, \& MacLean, 1989; Jonsson, Rosengren, Dotevall, Lappas, \& Wilhelmsen, 1999; Landsbergis, Schnall, Deitz, Warren, Pickering, \& Schwartz, 1998).

Several recent studies on work stress and BMI have found differential effects for BMI changes in men and women. In particular, Korkeila, Kaprio, Rissanen, Koskenvuo, and Sorenson (1998) investigated the effect of stress on future weight 
gain and suggested that higher levels of stress increase the likelihood of weight gain, with a greater effect seen in men than in women. More specifically, the researchers found that elevated levels of stress at baseline predicted a weight gain of 10 kilograms over a period of six years in men, but not in women. Similarly, Kivimaki and colleagues (2006) examined the association between work stress and BMI utilizing data from the Whitehall II study and found that high job demands and low control increased the likelihood of weight gain over five years in men with a "high" baseline BMI (BMI $\left.>27 \mathrm{~kg} / \mathrm{m}^{2}\right)$, and weight loss in males with "lean" BMI $(\mathrm{BMI}<22$ $\mathrm{kg} / \mathrm{m}^{2}$ at baseline), but found no corresponding bidirectional effect among women. Netterstrom and colleagues (1991) investigated the relationship between CHD risk factors (one of which was BMI) and job stressors based on the JD-C model in a two-part study on a sample of approximately 1,500 individuals from 11 Copenhagen municipalities. The participants were asked to complete a survey on previous and current illnesses, symptoms, exercise habits, smoking habits, family medical history, use of medicine, working conditions, and family situation. The participants were then asked to fast for several hours and participate in a health assessment where their blood pressure, weight, and height were measured and blood sample was taken. The researchers categorized job strain according to both subjective and objective classifications. The subjective classification was based on the participants' responses to survey statements regarding levels of demands and control in their jobs, whereas the objective classification was based on their job titles and modes of payment. Results indicate that more women than men were classified as having high strain jobs. After adjusting for age and sex, results showed 
that BMI was higher in the groups with self-reported low control and high demands, but the differences were not significant. However, there was a significant association between BMI and objective job strain (Netterstrom, Kristensen, Damsgaard, Olsen, \& Sjol, 1991).

Additionally, Overgaard, Gyntelberg and Heitmann (2004) conducted a systematic search of the studies that have investigated the relationship between job stressors and body weight, and found that, in total, only ten articles fulfilled the criteria. In general, the associations between job stressors and general obesity and abdominal obesity were found to be inconsistent. Six studies examined associations between BMI and job control; however, only one found a significant positive association (Georges, Wear, \& Mueller, 1992). And only one out of seven studies found a significant association between BMI and objective job strain (Netterstrom et al., 1991).

Most recently, Nyberg and colleagues (2012) pooled data from 13 independent cohort studies, resulting in an individual-level meta-analysis of 161,746 men and women, in their attempts to investigate the relationship between job stress and BMI. Consistent with results found by Kivimaki and colleagues (2006), results indicate a "U-shaped" association between job stress and BMI, such that the greatest risk of job stressors was evident in the underweight and obese groups, while the normal weight group experienced the lowest risk. The researchers also point out that despite the relatively modest associations that both weight gain and weight loss have with the onset of job stressors, they found little direct evidence linking job stressors to change in BMI, suggestive of the fact that it is unlikely that 
interventions targeted at reducing job stressors alone would be effective in combating obesity at the population level. The researchers also noted that the association was significantly reduced after adjusting for socio-economic status (SES), and that baseline job strain was not predictive of change in BMI in longitudinal analyses. These results fall in line with previous meta-analytic studies (Wardle, Chida, Whitaker, Gibson, \& Steptoe, 2011), as well as cross-cultural studies on Japanese (Ishizaki, Nakagawa, Morikawa, Honda, Yamada, \& Kawakami, 2008), Swiss (Berset et al., 2011), Swedish (Eek \& Ostergren, 2009), and Finnish samples (Kivimaki et al., 2002), which showed no reported association between job stressors and change in adiposity.

Despite the mixed results cited, this study makes the proposal that job stressors contribute to one's levels of job satisfaction as well as his or her BMI levels. On the basis of the JD-C model (Karasek, 1979) and the previously mentioned research, it is anticipated that jobs with high psychological demands and little opportunities for how and when to accomplish one's work contribute to lowering employees' overall experience of job satisfaction. Additionally, I anticipate high job demands and low control to be associated changes in one's BMI.

Both of these are expected to occur as a result of rising levels of frustration with one's supervisors and coworkers, feelings of helplessness from not being able to accomplish one's workload, one's inability to decompress or even sleep at night, and the triggering of negative coping mechanisms, such as 'stress eating' (Payne, Jones, \& Harris, 2005). For example, researchers have looked at changes in eating behavior (eating "comfort food" and lower consumption of vegetables) due to the 
expectation that eating may alleviate feelings of stress and job strain by making one feel good (Tsutsumi et al., 2003). Stress-related eating is defined as trying to make oneself feel better by eating or drinking in a stressful situation (Kouvonen, Kivimaki, Cox, Cox, \& Vahtera, 2005; Laitinen, Ek, \& Sovio, 2002). Torres and Nowson (2007) posit that the appetite suppression (under eating) that characteristically occurs when an acute stress is experienced (i.e., a threat to personal safety) and the instant physiologic "flight or fight" response that an individual undergoes is different than the response to exposure to chronic psychological stressors, such as job pressures. The researchers argue that under prolonged work duress the typical response may not be to avoid food, but rather to seek out and consume energy-dense, calorieladen, and sugary foods (over eat), which may overtime impact one's BMI as well as how satisfied one is with their job if all they see if that their job is the source of their stress and the root of their negative behaviors. Stress eating may very well be utilized as a coping mechanism in that individuals may choose to eat too much food or make unhealthy food choices. Also, on the basis of the Ego Depletion Model (Baumeister et al., 1998; discussed in more detail in the next chapter), if a person's self-regulatory/coping resources are depleted due to job stressors, it is that much easier to make the unhealthy food choice than it is to make the healthy one (i.e., making the decision to eat a chocolate bar or some other unhealthy food because you don't have the self-regulatory resources to stop yourself).

Though it makes plausible sense that high demands and low control at work could contribute to changes in BMI, the empirical evidence far from substantiates that notion (Berset et al., 2011; Kivimaki et al., 2006; Nyberg et al., 2012). As such, it 
is critical that researchers investigate beyond just the direct effects in search of other possible mechanisms linking stressors at work with adverse changes in one's weight. Not only would this understanding increase our awareness of the development of diseases that are related to body weight, but it would also help with the development of targeted and preventative interventions on modifiable behaviors (Berset et al., 2011). Accordingly, the following chapter discusses possible mediators in the job stressor-employee health relationship, with a particular focus on exercise. 


\section{Chapter III: Exercise as a Mediator}

The purpose of this chapter is to further discuss the possibility that the mixed results of the relationship between job stressors and BMI is suggestive of the notion that it may in fact be mediated through another relevant factor. As Nyberg and colleagues (2012) have already suggested that interventions will have to focus on factors other than just reducing job stressors in the workplace in order to be successful, researchers have followed suit by beginning to examine the mediating role of physiological pathways and health behaviors. A brief discussion of both, with a particular focus on exercise as the specific health behavior of interest in this study, is provided in this chapter. The Ego Depletion Model (EDM; Baumeister et al., 1998) is also introduced as the theoretical underpinning for exercise as the mediator between job stressors and job satisfaction and BMI. Physiological Mediators

With regards to physiological factors, researchers have typically studied changes in stress hormones, namely cortisol, epinephrine, norepinephrine and interlukin-6, as the first phase/ initial responses triggered in the central nervous system to prepare an organism to cope with demands that threaten to disrupt its homeostatic systems (Fox et al., 1993; Ganster, Fox, \& Dwyer, 2001; Ganster \& Rosen, 2013). The allostatic load model (Juster, McEwen, \& Lupien, 2010; Lupien et al., 2006; McEwen \& Stellar, 1993) posits that 'allostasis' is the process of adjustment of various effector systems (cardiovascular, neuroendocrine, and others) that serve to cope with real, imagined, or anticipated challenges to 
homeostatic systems (Ganster \& Rosen, 2013). The hypothalamic-pituitary adrenal (HPA) axis, which secretes cortisol, is an example of an allostatic system that operates around certain established points, which fluctuate as a result of circadian or seasonal rhythms. These set points, however, can also be reprogrammed after continual exposures to chronic demands that continually push them beyond their normal ranges.

Research has shown that among white-collar workers who are mainly exposed to mental demands, epinephrine levels are raised about $50 \%$ at work compared with non-work conditions (Lundberg \& Johansson, 2000). The researchers also found that among blue-collar workers performing manual tasks and being physically active that epinephrine increased by $100 \%$ and that norepinephrine, which is involved in blood pressure homeostasis, increased by $50 \%$. Whereas epinephrine levels increase in response to both pleasant and unpleasant stimulation, cortisol levels seem to be more sensitive to negative emotional conditions. Elevated cortisol levels and, subsequently, the over-activity of the HPA axis has been associated with increased risk of cardiovascular disease, accumulation of fat in the abdominal region, Type 2 diabetes, reduced immune functioning, and cognitive impairment (Lundberg \& Johansson, 2000). Kristenson and colleagues (1998) found that individuals exposed to chronic psychosocial stress displayed attenuated cortisol responses combined with elevated baseline levels. Additionally, Ganster, Fox, and Dwyer (2001) found that cortisol levels after work mediated the effects of the demands-control interaction on health care costs. These bodily responses are important for our successful coping to acute physical threats. 
In our modern world, however, stress is more often psychological and psychosocial in nature rather than physical, which means that some of these bodily responses may give rise to harm rather than protection (Lundberg, 2005).

\section{Health Behaviors}

In addition to physiological pathways, researchers are also investigating the role of health behaviors, which are behaviors that represent intentional and unintentional actions that individuals engage in to maintain or change their current health status (Kasl \& Cobb, 1966; Stone, 1979 in Robbins et al., 2012). Typically, poor health behaviors include smoking (Elovainio, Kivimaki, Puttonen, Lindholm, Pohjonen, \& Sinervo, 2006; Kivimaki, Ferrie, Brunner, Head, Shipley, Vahtera, \& Marmot, 2005), excessive alcohol consumption (Rospenda, 2002), eating a high caloric diet (Kouvonen et al., 2005; Torres et al., 2007), leading a sedentary lifestyle/low levels of physical activity and exercise (Brisson et al., 2000; Elovainio et al., 2003), and decreased sleep time (Elovainio et al., 2003).

Not surprisingly, Pratt (1971) found that a higher quality of personal health behaviors and practices (e.g., sleep, exercise, dental hygiene, nutrition) was related to a higher subjective level of health and to fewer health problems. Lundberg (2005) notes that the human body is rather resilient and can resist short-term demands, but that we need periods of rest and recuperation to activate anabolic processes (repair, healing, and growth). The researcher suggests that short-term periods of recovery may encompass taking a lunch break, an evening rest, a weekend off, or even a vacation. Two additional contributors to anabolic effects are regular moderate physical exercise and sleep (Akerstedt, 1997). Empirical evidence is also 
suggestive that the costly toll of many health problems can be reduced through a healthy diet, regular exercise, proper sleep and rest habits, restraint from smoking and alcohol or substance abuse, and reduced risk-taking behavior (Matarazzo, 1982; Pratt, 1971; Williams \& Wechsler, 1972). It follows then that one's health behaviors may be negatively impacted by stress. Interestingly, Langlie (1977) found that subjects with many demands on their time reported feeling a lack of control and perceived the costs of maintaining good health practices as high, indicating that health behaviors may be adversely modified by stressful situations and high stress jobs. With evidence that stress affects health behaviors that, in turn, influence one's health status, we can hypothesize that health behaviors may mediate the relationship between job stressors and health.

One health behavior in particular that has been highlighted in research as a key behavioral factor leading to obesity is reduced physical activity or lack of exercise (Kouvonen et al., 2005). It may be worth noting that while the terms physical activity, physical fitness, and exercise are often used interchangeably, they have separate and distinct meanings according to the U.S. Department of Health and Human Services (DHHS, 2002). For example, physical activity is often categorized by the context in which it occurs, such as leisure time, transport, household, and occupation. It can be defined as "bodily movement produced by the contraction of skeletal muscle that increases energy expenditure above the basal level" (DHHS 1996, 2002). Physical fitness, on the other hand, can be used to describe athletic-, performance- or health-related fitness and is defined as the "ability to carry out daily tasks with vigor and alertness, without undue fatigue, and with ample energy 
to enjoy leisure-time pursuits and to meet unforeseen emergencies" (Park, 1989). Attributes of physical fitness include cardiorespiratory endurance; flexibility; balance; body composition; and muscular endurance, strength and power.

Lastly, exercise is considered a subcategory of physical activity and has been defined as "physical activity that is planned, structured, repetitive, and purposive in the sense that improvement or maintenance of one or more components of physical fitness is the objective" (Caspersen et al., 1985). For the purposes of this study, the term exercise was used to maintain consistency with how the survey questions were worded. Although there is a big difference between being active at work versus how often one exercises during one's day, the terminologies were likely perceived by the study participants to be interchangeable given the nature of the context within which they work (out in the field several days per week).

Although the direct association between job strain and cardiovascular disease risk factors (one of which is BMI) has long been debated, Nyberg and colleagues (2013) recently conducted a meta-analysis on 47,000 men and women and found that job strain affects cardiovascular disease risk factors via two ways: 1) poor health behaviors (one of which was physical activity) and 2) increasing risk of diabetes (glucose levels). The researchers' findings also corroborate those found by the IPD-Work consortium on the largest data set to date $(\mathrm{N}>170,000)$ on work characteristics (Fransson, Heikkila, Nyberg, Zins, Westerlund, et al., 2012), which has shown that job strain more strongly predicts physical inactivity than physical inactivity predicts job strain. Fransson and colleagues (2012) also found robust cross-sectional and prospective associations between unfavorable work 
characteristics (high demands/low control) and leisure-time physical inactivity, with $21 \%-26 \%$ higher odds for inactivity among participants working in high strain and passive jobs compared with those in low-strain jobs. The relative importance of studying health behaviors such as physical activity is that it is a modifiable behavior, as opposed to factors which individuals have little or no control over, such as sex, intrauterine and childhood environment, or family history (Kujala, Kaprio, Sarna, \& Koskenvuo, 1998). Accordingly, Nyberg and colleagues (2013) have suggested that risk management among people with job stress should focus on glucose levels and lifestyle factors, especially exercise.

Among its myriad benefits, physical activity and fitness are believed to reduce premature mortality (Fransson et al., 2012; Kujala et al., 1998). Decades of research have also found exercise to be a protective means against elevated stress reactivity by suggesting that it helps the body regulate the hypothalamic-pituitaryadrenal (HPA) axis and the sympathetic nervous system (SNS), both of which play significant roles in the body's response to physiological stress (Ramirez \& Wipfli, 2013). Not only has research shown a delayed HPA axis response to stress in animals after physical activity, but it has also shown that exercise-trained individuals exhibit an attenuated HPA axis response to physical and mental stress (Luger, Deuster, Kyle, et al., 1987).

Thus, for the purposes of this study, exercise will be investigated as a possible mediator in the job stressor-employee health relationship. Because I don't expect exercise to be the only explanation for the existence of such a relationship, I propose exercise as a partial mediator that may account for some but not all of the 
variance in the relationship between job stressors and BMI/job satisfaction. Exercise as a Partial Mediator in the Job Stressors-BMI Relationship

Not surprisingly, studies that the have investigated both BMI and exercise have yielded mixed results. In support of an existing relationship, Kujala and colleagues (1998) conducted a longitudinal study from 1977 to 1994 on 7,925 healthy men and 7,977 healthy women of the Finnish Twin Cohort aged 25 to 64 years who responded to a questionnaire on physical activity habits and known predictors of mortality (one of which is BMI). Those who reported exercising at least six times per month with an intensity corresponding to at least vigorous walking for a mean duration of 30 minutes were classified as "conditioning exercisers", whereas those who reported no leisure physical activity were classified as "sedentary", and those with other intermediary responses were classified as "occasional exercisers." Results indicate that after adjustment for age and sex, both "occasional exercisers" and "conditioning exercisers" had reduced risks of mortality compared with those who were "sedentary", pointing to the beneficial effect of physical activity after controlling for other predictors of mortality.

Additionally, a recent meta-analysis by Conn and colleagues (2009) on the effects of workplace physical activity interventions on work attendance, job satisfaction, and job stress indicate small but positive effect sizes for anthropometric measurements (one of which was BMI), indicating better scores following interventions among the treatment subjects. The raw mean difference between the treatment and control groups in terms of BMI was -.3, which signifies that if the post-intervention BMI mean for the treatment group was 25.0, then the 
control group had a mean BMI of 25.3. One interesting meta-analytic result related to anthropometric effect sizes was the fact that intervention delivery at the worksite yielded a much larger mean effect size (.17) than in those delivered elsewhere (.05). Additionally, interventions delivered on paid company time versus outside of work hours were significant for fitness (.92 vs .49) and anthropometric measures (.22 vs .02). Moreover, results indicate that workplace participation in designing the interventions, as compared to interventions designed by people outside of the organization, was significant for fitness (1.18 vs .49) and anthropometric outcomes (.22 vs .06), but there were no significant differences for lipids or physical activity behaviors. Lastly, studies with onsite fitness facilities reported larger mean effect sizes on lipids (.32 vs .07) and anthropometric outcomes (.24 vs .05), but there were no significant mean effect size differences for fitness or physical activity behavior. The researchers caution against relying too heavily on the significance of the results given the limited number of studies with sufficient data to calculate effect sizes and adequate heterogeneity among studies, in addition to the fact that physical activity was rarely measured objectively, leading to difficulty in comparisons across interventions. Despite these mixed results on improved work attendance, job satisfaction, and job stress, the researchers did want to stress that even modest reductions in absenteeism, for instance, may result in substantial fiscal savings when multiplied by many employees.

Other studies have found no association between physical activity and BMI. For example, Brisson and colleagues (2000) examined the associations between psychosocial factors at work (job demands and job control), sedentary behavior and 
BMI in a study population composed of 3,531 men and 3,464 women employed as white-collar workers in 21 organizations. Results provide partial support for an association between psychosocial factors at work and prevalence of sedentary behavior. Specifically, prevalence of sedentary behavior was elevated in men in the lowest quartile of job control, who were also in the passive, high demands group. In women, this prevalence was elevated in the highest quartile of job demands. However, there was no association between psychosocial factors at work and BMI, nor was there a relationship between prevalence of sedentary behavior and BMI.

Elovainio and colleagues (2003) investigated physical activity as a potential mediator between organizational justice and health outcomes. The researchers asked participants a series of questions related to their overall physical activity levels, including weekly frequency, mean duration, and mean intensity of the physical activity sessions. Those reporting less than half an hour of fast walking per week were considered sedentary. Contrary to the proposed hypotheses, exercise was not found to be a significant mediator in the relationship between organizational justice and health outcomes.

\section{Ego Depletion Model}

Engaging in exercise requires time and energy, two resources that tend to suffer under duress (Sonnentag \& Jelden, 2009). That being the case, jobs with high demands and low control are believed to drain individuals of their psychological and physical resources that would normally be devoted to improving their health and well-being (Hammer \& Sauter, in press). Specifically, high demands and low controls, as well as other job characteristics, such as long hours and inflexible 
schedules, may deplete one of the time, energy, strength and goodwill they would typically need to engage in physical activity. As such, the Ego Depletion Model (EDM; Baumeister, Bratslavsky, Muraven, \& Tice, 1998) is presented as the theoretical pathway via which job stressors impact the available resources that one has to engage in physical activity, which would, in turn, be expected to influence employees' levels of job satisfaction and their BMI.

The EDM suggests that humans struggle to self-regulate, which involves the capacity to exert self-control in order to override and alter undesirable responses (Baumeister, Heatherton, \& Tice, 1994; Baumeister et al., 1998). The ability to abstain from gratifying one's immediate needs is extremely adaptive and enables people to engage in goal-directed behavior to obtain long-term desirable outcomes (Baumeister, 2005). Hence, people able to exert self-control over a particular behavior or action are more likely to successfully execute those behaviors.

Self-regulation is governed by a limited resource that allows people to control their impulses and desires, but that is expended when people engage in behaviors that require self-control. Although the exact nature of this resource remains unclear, the EDM model conceptualizes self-regulation as operating much like a 'muscle' (Muraven, Tice, \& Baumeister, 1998), such that any regulatory act depletes this muscle, leaving fewer resources available and decreasing one's strength for subsequent regulatory tasks (Muraven \& Baumeister, 2000). Just as a muscle loses its strength and becomes fatigued after periods of exertion, our selfcontrol resources become depleted after engaging in actions that require selfregulation with insufficient opportunity for recovery (Hagger, Wood, Stiff, \& 
Chatzisarantis, 2010). Hence, ego-depletion occurs when the depletion of one's selfcontrol resources leads to a failure in self-regulation (Baumeister et al., 1998). Accordingly, several researchers have identified lapses in self-regulation as an important psychological determinant in health-related behaviors, including exercise (Dishman, 1994; Dishman, Ickes, \& Morgan, 1980). Adherence to exercise constitutes a prime example of a behavior that requires people to exert self-control, where failure to self-regulate results in lapses in one's adherence to and continuation in an exercise regimen (Hagger et al., 2010).

Baumeister and colleagues (1998) found that when participants were asked to perform one act that did not result in immediate pleasure, their performance on subsequent acts requiring self-restraint or self-regulation was diminished. In a series of experiments, researchers have noted that the limited self-regulatory resources that one has can be either depleted through use, replenished by experiencing positive emotions (Tice, Baumeister, Schmueli \& Muraven, 2007), or increased through small exercises of self-control (Muraven, Baumeister \& Tice, 1999). Researchers Hagger and colleagues (2010) posed the scenario where an office employee that is prescribed an exercise regimen by his physician is required to write a report at work that is due by the end of the day. The report requires a large number of laborious calculations, which ultimately requires a considerable amount of self-control resources in order for the employee to resist the urge to stop working on it. Moreover, the employee's self-regulatory resources are further depleted if he feels he is unable to take a break out of fear and anxiety that he won't meet his deadline. At the end of the day when it comes time for the employee to 
participate in his physician-prescribed exercise session, the employees decides to not go exercise and instead opts to go for a drink and a meal with friends. According to the EDM, not only did the employee avoid engaging in exercise, a task that was likely to demand too high of resources, but he was also unable to resist the temptation of the more appealing option (drinks and bar food), which require little to no self-control demands. Importantly, the researchers point out that the reason behind the lapse was not physical tiredness per se, so much as the depletion of selfcontrol resources which likely led to the employee's mental fatigue (Hagger et al., 2010).

Whether it is refraining from eating or drinking too much, saving instead of spending, finishing one's work instead of daydreaming, or going for a jog instead of watching another sitcom, failures to regulate one's own behavior are common, sometimes leading to devastating consequences. For instance, regulatory failures have been linked to substance abuse, criminal behavior, sexually transmitted diseases and consumer debt (Baumeister et al., 1994; Muraven \& Baumeister, 2000). It is therefore essential to understand the processes of self-control and the parameters that determine its success. Bearing the concept of self-regulation in mind, time and energy may be viewed as essential resources not only for completing job tasks, but also for engaging in physical activity, especially if one works in a job with high demands and low control. Research has demonstrated that depletion in one's self-regulatory resources can lead to reduced participation in exercise (Oaten \& Cheng, 2005). Therefore, it follows that everyday demands that tax employees' finite self-control resources, such as coping with job stressors, may compromise 
their self-regulatory capacity to participate in a regular health-related exercise (Hagger et al., 2010; Neubach \& Schmidt, 2008; Wallace, Edwards, Shull, \& Finch, 2009). Further, in returning to the muscle analogy, researchers have shown that a recovery period is necessary for full self-regulatory capacity to be restored. For example, Tyler and Burns (2008) found that for brief self-control tasks, a 10-minute break was sufficient to almost completely restore individuals' self-regulatory capacities. Oaten and Cheng (2006), however, found that a 45-minute break only resulted in partial recovery for individuals performing prolonged initial depleting tasks. Together these results indicate that recovery is more complete when longer periods of recovery are provided. This is important for prospective exercisers to consider, as it is likely that fully replenished self-control resources will maximize the probability that employees will be able to engage in exercise, and subsequently, exert the self-control to engage in a follow-up exercise session (Hagger et al, 2010).

Preliminary research on increased job demands and spillover between work and non-work life (Hammer, Cullen, Neal, Sinclair, \& Shafiro, 2005a; Totterdell, Spelten, Smith, Barton, \& Folklard, 1995) has shown that high-strain work environments can contribute to weight gain and obesity if work schedules are so demanding that individuals don't have time to be physically active (due to long work hours or commute times), or if workloads are so high that employees don't have time to prepare and/or eat healthy meals (Kouvonen et al., 2005; Robbins \& Fray, 1980). This falls in line with Langlie's (1977) sentiment that individuals with many demands on their time reported feeling a lack of control and perceived the costs of maintaining good health practices as high. This is also a testament to the fact that 
when time falls short, something has to give, and unfortunately, that usually results in poor health behaviors, such as choosing to forego physical activity.

Therefore, based on the premise of self-regulation, it follows that increased job stress would lead to a drain in one's resources, which would lead to negative health behaviors, such as the choice to forego exercise, and that, in turn, may lead to a change in one's weight and BMI, as well as their decreased psychological wellbeing over time. Considering the wide reaching impact that job stressors have on one's health, both on and off the job, the mediating effect of exercise, even if only the partial effect, is important to study because a significant change in one's BMI and job satisfaction levels can have deleterious implications not only for one's quality of life outside of work, but also to the overall functioning of the organization with regards to the employee's level of productivity and mental focus (Searle, Bright, \& Bochner, 1999), getting hurt on the job and the costs incurred via increased worker's compensation claims (Jex \& Crossley, 2005), absenteeism and subsequent turnover if the employee is no longer physically able to perform the job and/or has lost all motivation to perform the job (Colquitt, 2001).

The manifestation of such a progression of events may be gradual, but a change in one's health behaviors (lack of physical activity) and a subsequent change in one's BMI may be one of the first signs that some aspect of that individual's life should change before it's too late. An intervention targeted at addressing one's health behaviors may actually be an initial necessary step to the prevention of far worse health outcomes related to increased BMI, such as increased blood pressure, Type 2 diabetes, CHD, or even mortality (Kivimaki et al., 2006). In fact, research has 
shown that even relatively small changes in physical activity levels can play an important role in weight management and the reversal of obesity trends (DHHS, 2002). Therefore, it follows that an employee's high stress job will affect his physical health via the behaviors they engage in or choose to avoid. Thus, in addition to the direct effect that job demands and job control have on BMI and job satisfaction, this study also aims to investigate the effects that exercise has in partially mediating the relationships between job demands and job control on BMI so that interventions can be implemented to help individuals become aware of and deal with their modifiable behaviors long before chronic and fatal outcomes develop.

\section{Exercise as a Partial Mediator in Job Stressor-Job Satisfaction Relationship}

From a public health perspective, physical activity has long been documented as being beneficial for offsetting CHD risk and disease prevention as well as promoting psychological well-being, namely warding off depression, lowering anxiety, and enhancing life satisfaction (Rethorst et al., 2009). Studies of its relationship with job satisfaction, however, are not as pervasive in the field and, like BMI, the results are far from conclusive. For example, Kirkcaldy and colleagues (1994) conducted a large-scale study of leisure habits and satisfaction on 533 senior police officers (aged 28-58 years) from the UK and found that exercisers had a higher level of job satisfaction and better physical and mental health than nonexercisers. However, the findings also revealed that the perceived stress of police work was not related to exercise habits, and exercise was not found to be a buffer between stress and job satisfaction or personal well-being. Although exercise did not moderate job satisfaction, the researchers posited that Type A personality and 
locus of control might be expected to influence job stress and thus job satisfaction. Similarly, Altchiler and Motta (2006) conducted a study on the effects of aerobic and nonaerobic exercise on job satisfaction, and it was discovered that aerobic exercise is superior to nonaerobic exercise for anxiety reduction, however no changes were evident for job satisfaction, absenteeism, or resting heart rate.

In support of the relationship between physical activity and job satisfaction, Daley and Parfitt (1996) examined differences in mood states, physical well-being, job satisfaction, and absenteeism in members, non-members, and individuals on the waiting list for a British corporate fitness club. The sample consisted of 293 head office employees from a leading British food retail company. Results from the study suggest that club members have better psychological mood states and physical wellbeing than non-members. More importantly, results show that members were more satisfied with their jobs and took fewer sick days than non-members.

Conn and colleagues (2009) found mixed meta-analytic results for the effects of physical activity on job stress, job satisfaction and work attendance. Results show that job satisfaction was significantly greater among treatment subjects than controls following interventions in the two-group pre-post effect analysis (effect size $=.20$ ), but similar findings did not approach significance for the two-group posttest analysis. Additionally, the researchers cautioned against drawing too much information from their findings given the small number of studies $(\mathrm{k}=5)$ that reported job satisfaction as a variable of interest, but noted that this is a start for researchers and practitioners alike.

While narrative reviews and cross-sectional studies of the effects of exercise 
on stress reactivity have found conflicting results (de Geus, Van Doornen, De Visser, \& Orlebeke, 1990; Taylor, 2000), several recent meta-analyses have found significant improvements in stress reactivity after some form of physical activity (Alderman, Rogers, Landers, \& Arent, 2004; Crews \& Landers, 1987; Ramirez \& Wipfli, 2013). In fact, meta-analytic results from the analysis of randomized controlled trials with a large sample of participants $(\mathrm{N}=1,252)$ found an effect size of -.31 , presenting reliable evidence for the use of exercise to reduce one's reactivity to psychological stressors (Ramirez \& Wipfli, 2013). Additionally, Wipfli and colleagues' (2008) meta-analysis on randomized trials and dose-response analyses also found that, in general, exercise groups showed greater reductions in anxiety compared with groups that received other forms of anxiety-reducing treatment. Although there are no known meta-analytic studies on the effects of exercise on job satisfaction to date, it is anticipated that its effects would be similarly beneficial as it is on anxiety, depression, and overall stress reactivity.

With fairly strong evidence showing that exercise can help alleviate anxiety and depression, numerous psychological and physiological theories have been proposed. Though the majority have been tested and supported primarily by animal models, they are purported to relate to humans as well. Landers and Arent (2001) have summarized the literature. Some of the psychological theories still under investigation include increases in self-esteem, self-concept, and self-efficacy. Physiological hypotheses that have been developed to explain the anxiolytic and anti-depressive effects of exercise include the brain-derived neurotrophic factor hypothesis, the endorphin hypothesis, the endocannabinoid hypothesis, the 
hypothalamic-pituitary-adrenal axis hypothesis, and the norepinephrine hypothesis (Landers \& Arent, 2007). In the case of job satisfaction, it follows that a similar feedback loop would exist whereby individuals that engage in exercise would also experience increased levels of job satisfaction as a result of increased endorphins, norepinephrine and serotonin levels, improved body image, and/or an increased sense of mastery and self-esteem.

In addition, based on the premise of self-regulation and the existing literature on the antidepressive benefits of exercise (Rethorst et al., 2009), it also follows that a hard working employee with reported levels of increased job demands and a lowered sense of control over one's schedule (effort reward imbalance) is expected to feel drained of his or her resources (ego depletion model), which would lead to negative health behaviors, such as an individual's decision to forego exercising, and that, in turn, may lead to a change in one's psychological wellbeing (lowered job satisfaction) over time, especially if they attribute the guilt and blame of not working out on their job.

Considering the wide reaching impact that job stressors have on one's health, both on and off the job, it would behoove employers to increase their awareness of the impact that a health behavior, such as exercise, has on employees' levels of job satisfaction, as studies have shown that job dissatisfaction is related to lowering of one's performance, and increasing absenteeism, counterproductive work behaviors, turnover intentions, and ultimately, turnover (Colquitt, 2001). As such, in addition to the direct effect that job demands and job control are anticipated to have on job satisfaction, this study also aims to investigate the effects that exercise has in 
partially mediating the relationships between job demands and job control on job satisfaction so that we can better understand the intervening function of modifiable behaviors on individuals' mental well-being in the workplace.

Moderators in the Job Stressors - Health Relationship

Going beyond the exploration of factors that mediate the job stressoremployee health association, researchers are also seeking factors that buffer that relationship. Some of the myriad factors that have been investigated as moderators to this relationship include social support, job control, locus of control, coping styles, self-efficacy, personality (Type A) and other individual attributes (hardiness), though the results have been somewhat mixed (Bosma et al., 1997; Bosma et al., 1998; Karasek \& Theorell, 1990; Kivimaki, Ferrie, Shipley, Gimeno, Elovainio, Vogli, Vahtera, Marmot, \& Head, 2008; Sonnentag \& Frese, 2003).

In light of the inconclusive evidence, Karasek and Theorell (1990) have vehemently argued that "decades of research have still not identified "the personality variables" resistant to job strain (p. 96), pointing to the fact that personal characteristics may only play a modest role in the relationship between job strain and employee health, but that perhaps the main emphasis of the relationship may lie in the environment, and in particular, the stressors in one's work environment (Schnall et al., 1994). In observance of this call to action, this study examines organizational justice, or fairness of treatment within the work context, as a possible moderator in the relationship between job strain and employee health (Robbins et al., 2012). 


\section{Chapter IV: Organizational Justice as a Moderator}

The purpose of this chapter is to provide a brief overview of organizational justice and its widely accepted dimensions of distributive and procedural justice after setting the stage for it as a viable moderator in the job stressor-employee relationship. Next, I will introduce the Relational Model (Lind \& Tyler, 1992) and the Effort-Reward Imbalance Model (ERI; Siegrist, 1996) as the theoretical explanations for understanding why procedural and distributive justice in the workplace are related to employee well-being (job satisfaction) and health outcomes (BMI). Setting the Stage for Organizational Justice as a Moderator

With the changing nature of our work environment and workplace demands, it is possible to see how working a high stress job, day in and day out, may very well be a factor that sets a negative cycle into motion. A cycle that can't very easily be stopped, especially if an employee feels that his supervisor is partly to blame for creating unrealistic workload expectations and breeding a climate where workplace concerns can't be discussed (Kelloway, Sivanathan, Francis, \& Barling, 2005; Thomas \& Ganster, 2005).

Organizational justice has proven to be a catalyst behind a number of different workplace studies (Colquitt, 2001; Colquitt et al., 2001), and has been reported to have an impact on the health of employees as well (Elovainio, Kivimaki, \& Vahtera, 2002; Kivimaki, Elovainio, Vahtera, Virtanen, \& Stansfeld, 2003; Taris et al., 2002; Tepper, 2001). Core to these research findings is the notion that perceived unfair treatment due to increased job stressors (i.e., in the form of strenuous job 
demands or poor reciprocity between the costs and returns of a job) impacts psychological stress reactions, resulting in poorer chronic psychological well-being and health (Sonnentag \& Frese, 2003). And as mentioned previously, Cropanzano and colleagues (2005) have suggested that mitigating stressful situations rooting from injustice at work is one of the viable ways for management to address employee's health concerns, especially considering the amount of time that employees spend in direct contact with their supervisors and the dependence on them for information, rewards and resources. Thus, not surprisingly, researchers have noted that it could be fruitful to explore organizational justice as a possible resource against the long-term harmful impact of workplace stressors (Elovainio et al., 2001; Robbins et al., 2012).

According to Elovainio and colleagues (2001), this broadened focus of justice research on health outcomes is important for two reasons. First, perceptions of fairness provide employees a sense of predictability in and control over their work environment, and thus, have been shown to be associated with increased job control, one of the main components of job strain, as well as a one of the chief psychological predictors of employee health (Bosma et al., 1997; Bosma et al., 1998; Karasek, 1990; Kivimaki et al., 1997). Second, in light of the social and interpersonal nature of justice, in particular procedural justice (Thibault \& Walker, 1975), perceptions of fairness are related to employees' levels of trust in those responsible for making important decisions about their workplace situations. Moreover, it is believed that employees who perceive their organization to be fair can ultimately trust that the organizational decisions made are reached via a fair and transparent 
manner, thereby reducing employees' feelings of mistrust, stress, and uncertainty (Robbins et al., 2012).

The real significance behind these two stated reasons is that perceived lack of fairness and mistrust can be characteristic of individual's attributes, but it can just as well be a characteristic feature of a particular environment; both of which have been found to predict morbidity and mortality (Elovainio et al., 2001). Despite these significant implications, however, published research focusing on the relationship between organizational justice and health has been limited (Robbins et al., 2012). While many studies investigate lack of fairness as a predictor of strain, and subsequently health, few studies to date have looked at justice as a potential buffer to the effects of a high strain job on psychological well-being and physical health (Brotheridge, 2005; Francis \& Barling, 2005; Tepper, 2001). Accordingly, this chapter will address the moderating effect of organizational justice, in particular distributive and procedural justice, in the relationship between job demands and job control with employee well-being (job satisfaction) and physical health (BMI). Overview of Organizational Justice

Given the vital role that justice plays in the various areas of work, it is not surprising that an explosion of research aptly labeled "organizational justice" emerged as an important contributor to the field of I/O psychology, human resources management, and organizational behavior (Hendrix, Robbins, Miller, \& Summers, 1998). Meta-analytic results highlight that all of the organizational justice dimensions are associated (to verifying degrees) with outcome satisfaction, job satisfaction, organizational commitment, trust, agent-referenced evaluation of 
authority, system-referenced evaluation of authority, withdrawal, negative reactions, organizational citizenship behaviors (individual and organizational), and performance (Colquitt et al., 2001). Organizational justice has been widely accepted in the extant literature as a multi-dimensional construct consisting of two dominant dimensions: distributive and procedural justice (Adams, 1965; Colquitt et al., 2001; Greenberg, 1993; 2004; Leventhal, 1976; Thibaut \& Walker, 1975; Tyler, 1994). Review of Distributive Justice

Distributive justice refers to the equitable allocation of rewards and punishments and is largely concerned with the fairness of tangible (i.e., pay) or intangible (i.e., praise) organizational outcome distributions in relation to individual or group inputs (Leventhal, 1976). Much of the distributive justice research is derived from Adams' equity theory (1965), which uses a social exchange theory framework (Blau, 1964; Thibaut \& Kelley, 1959) to evaluate fairness. Adams proposes that people are more concerned about the fairness of a particular outcome rather than the absolute level of the said outcome, such that people compare rewards for their own efforts or "inputs" (i.e., time, money, education, intelligence, experience) with the rewards others receive for their efforts. Thus, if the ratio between effort and reward is not proportional, inequity exists, increasing one's levels of dissatisfaction as the inequity becomes greater (Harris, 2000; Hendrix et al., 1998); conversely, equity results when the ratios are equivalent (Greenberg, 1990). Therefore, according to Adams' equity rule, an individual who has invested a large amount of input (e.g., time, money, energy) should receive more from the group than someone who has contributed very little. It follows then that members of 
large groups would prefer to base allocations of rewards and costs on equity rather than some other standard. Adams also proposed that evaluations of inequitable distributions produce negative emotions, which may motivate individuals to change their behavior or distort their cognitions associated with perceptions of unfairness (Adams, 1965; Gilliland, 1993).

Equality and need were identified as two additional standards ("allocation rules") by which fairness can be determined (Deutsch, 1975; Leventhal, 1976). The equality rule suggests that all individuals should have the same chance of receiving an outcome regardless of their inputs. Therefore, an individual that contributes 30\% towards the group's success should receive as much as someone who contributes $70 \%$. The needs rule, on the other hand, states that those in greatest need should be provided the resources regardless of their input. According to Gilliland (1993), a limiting factor of the needs rule, however, is the inevitable violation of the equality and equity rules. For example, providing resources to a disabled or disenfranchised individual simply because he or she is disabled or disenfranchised may override the fact that the individual hasn't contributed as much to the group goal (thereby violating the equity rule as a result of the fact that their efforts/inputs may be less than another individual's) or may prevent the other team members from receiving the resource, especially if the resource is a scarce commodity (thereby violating the equality rule if there isn't enough of a resource for all to have an equal share). Consequently, the inadequacies of equity theory as the sole conceptual framework for distributive justice shifted researchers' attention away from outcome distributions to a second wave of research called procedural justice. 
Review of Procedural Justice

Established in the mid 1970s as the application of "process control" in legal proceedings to the justice literature, Thibaut and Walker (1975) first introduced the concept of procedural justice in their published work summarizing third-party dispute resolution procedures as having both a process stage and a decision stage, whereby disputants are willing to give up control in the decision stage as long as control is retained in the process stage. This process control effect, often referred to as "fair process effect" or "voice effect" (Folger, 1977; Lind \& Tyler, 1988), suggests that disputants are more likely to view a procedure as fair if they perceived that they had process control throughout the legal proceeding (sufficient time to present their case, as well as control over the presentation of their arguments). The oftreplicated process control notion became synonymous with procedural justice and was eventually extended beyond the legal context to the interpersonal, educational and organizational settings (Colquitt et al., 2001; Folger \& Cropanzano, 1988; Leventhal, 1980; Leventhal, Karuza, \& Fry, 1980).

Applied to the organizational environment, procedural justice addresses the fairness of the processes that lead to decision outcomes and is concerned with the ways in which employees determine if they have been treated fairly in their jobs and, subsequently, the ways in which those determinations influence other workrelated variables, such as behaviors and attitudes toward the work, coworkers, and supervisors (Colquitt, 2001; Leventhal, 1980; Leventhal et al., 1980; Lind \& Tyler, 1988; Moorman, 1991; Thibault \& Walker, 1975). Greenberg (1990) noted that if people believe they are treated fairly, they would be more likely to hold positive 
attitudes about their work, their work outcomes, and their supervisors. This, in turn, would lead to increased satisfaction with team procedures (Thibaut \& Walker, 1975), higher likelihood of acceptance and adherence to general rules (Tyler, 1990), elevated satisfaction with the decisions resulting from the procedures (Greenberg, 1987), and an increased willingness to help their group (Tyler \& Degoey, 1995).

As a result of this dependence on the quality of one's social relationships with a decision maker as an important indicator of fair treatment, Bies and Moag (1986) suggested a separate and distinct type of justice acknowledging that people also consider interpersonal treatment to be a form of justice. The authors later retracted the position that interactional justice was a third type of justice in exchange for an explanation that it is a social form of procedural justice (Bies \& Moag, 1986; Colquitt et al., 2001; Cropanzano \& Greenberg, 1997).

Consequently, researchers have returned to the two-dimensional conceptualization of justice composed of outcome fairness (distributive) and process fairness (procedural), and suggest that interactional justice is merely a component of procedural justice (Colquitt et al., 2001). While all three justice dimensions contribute uniquely to fairness perceptions, meta-analytical results show that procedural and distributive justices were either the strongest or second strongest predictor of 15 different organizational outcomes, namely outcome satisfaction, job satisfaction, organizational commitment, and performance (Colquitt et al., 2001). Thus, for the purposes of this study, only distributive and procedural justice will be assessed. 
The Relationship Between Organizational Justice and Job Satisfaction

According to DeConinck and Stilwell (2004), understanding organizational justice is extremely important for organizations because of its relationship with employees' levels of job satisfaction and organizational commitment and, subsequently, their propensity to search for another job. Not surprisingly, the bulk of the organizational justice research focus has been placed on attitudinal and behavioral outcomes (Colquitt et al., 2001; Robbins et al., 2012), with satisfaction (be it with one's job, supervisor, coworkers or pay) being of particular interest to researchers (Jones \& Martens, 2007; Kim \& Leung, 2007; Simons \& Roberson, 2003; Sweeney and McFarlin, 1993). According to a meta-analysis by Colquitt and colleagues (2001), results have confirmed a strong, positive correlation between both distributive and procedural organizational justice and job satisfaction.

Research has typically found that individuals who perceive their levels of managerial treatment to be fair also experienced increased levels of job satisfaction (Cropanzano \& Folger, 1991; Elovainio et al., 2001). Schweiger and DeNisi (1991) found that perceptions of fairness and job satisfaction increased as did perceptions of a company's trustworthiness when supervisors provided open and honest communication during an imminent merger of two companies (a situation typically viewed as stressful and uncertain). McFarlin and Rice (1992) also found strong evidence that equity-based comparisons are important in determining one's level of satisfaction. More specifically, DeConinck and Stilwell (2004) found that distributive justice predicted pay satisfaction and procedural justice predicted supervisor satisfaction. These results suggest that when employees perceive that they are 
treated fairly in the amount of rewards allocated (distributive justice) and in the method by which they are allocated (procedural justice), they will be more satisfied with their pay and supervisor, respectively. McFarlin and Sweeney (1992), on the other hand, found distributive justice to be more predictive of personal outcomes (i.e., job satisfaction) and procedural justice to be a better predictor of organizational outcomes (i.e., organizational commitment).

With regards to change in justice perceptions over time, Ambrose and Cropanzano (2003) investigated faculty perceptions of tenure and promotion decisions and job attitudes three times over the course of two years and found overall support for the relationship between fairness perceptions and job satisfaction. More specifically, the researchers found that procedural justice was most influential prior to and soon after outcome decisions were made, whereas distributive justice was most influential to job satisfaction one year later. Furthermore, researchers found that candidates who perceive a promotional selection process to be fair also experience higher levels of job satisfaction (Folger \& Cropanzano, 1998b; Greenberg, 1990).

With more and more interest in cross-cultural studies as organizations expand into different regions of the world, Pillai and colleagues (1999) investigated the global effects of justice on non-Western cultures and found that procedural and distributive justice had significant and equally strong effects on job satisfaction across employee samples from Australia, Columbia, India, Jordan, and Saudi Arabia, in addition to the United States. Additionally, in a study on justice perceptions and job satisfaction among 137 Chinese workers employed in joint venture hotels, 
Leung, Smith, Wang, and Sun (1996) found a stronger association between procedural justice and job satisfaction than between distributive justice and job satisfaction, which tends to contradict the findings in North America (Folger \& Konovsky, 1989). Contrary to North American findings, Yoon (1996) examined the relationship between procedural and distributive justice and job satisfaction among 501 Korean workers and found that procedural justice had a stronger relationship with job satisfaction than did distributive.

All of the above mentioned research results provide ample support for the notion that perceived fairness is an imperative determinant of one's well-being within one's workplace environment whereby a majority of one's time is spent in direct contact with one's supervisor, whose fair treatment is responsible for a whole host of both positive and negative responses (Robbins et al., 2012), in particular their ratings of job satisfaction. Accordingly, this study will also investigate the relationship between the two dimensions of organizational justice (distributive and procedural) and the subjective well-being outcome of job satisfaction with the hope of providing additional empirical support to the I/O and OHP fields of study.

The Relationship Between Organizational Justice and BMI

Judge and Colquitt (2004) have explicitly argued that lowered perceptions of fairness and unfair treatment have a "visceral and even physiological effect" on employees, thereby disrupting their work attitudes and behaviors, as well as overall health. As fundamental as justice is in human societies and the ways in which we interact, evidence of its direct effect on our health, in particular BMI, is sorely lacking or has been poorly described in research (Ganster \& Rosen, 2013; Robbins et al., 
2012). High levels of justice are assumed to be related to reduced work stress, and thus related to a lowered risk of developing metabolic syndrome (one of which is BMI). Metabolic syndrome, in turn, is a cluster of risk factors for heart disease defined by at least three of the following: elevated blood pressure, elevated levels of fasting glucose and triglycerides, sex-specific low levels of high density lipoprotein cholesterol, and abdominal obesity as measured by waist circumference (Gimeno, Tabak, Ferrie, Shipley, De Vogli, Elovainio, Vahtera, Marmot, \& Kivimaki, 2010). Much of the work that has been published to date on the relationship between organizational justice and BMI (or objective health in general) has relied on data derived from the Whitehall II Study (Marmot, Smith, Stansfeld, Patel, North, Head, Brunner, \& Feeney, 1991), a prospective, observational, cohort study with eight data collection phases to date, spanning a total of 20 years of accumulated data on approximately 10,000 British civil servants (Marmot \& Brunner, 2005). The composition of the cohort, however, is mostly white, male, white-collar, office-based civil servants, and the justice measures utilized only assessed procedural and relational justice at phases I (1985-1988) and 2 (1989-1990), with the majority of the studies only investigating the relational dimension of justice. These data, however, have provided researchers the opportunity to more closely examine the association between perceived justice and morbidity and mortality. Additionally, there is cumulative evidence supporting the existence of a link from unfair treatment to experiences of strain and various health problems following prolonged stress exposure (Elovainio et al., 2001, 2002; Kivimaki et al., 2003; Kivimaki et al., 2002). 
Gimeno and colleagues (2010) explored the Whitehall II study data set and found that men who experienced a high level of workplace justice had a $25 \%$ lower risk of incident metabolic syndrome than employees with a low level of justice. The researchers also found that of the five metabolic syndrome components mentioned above, large waist circumference showed the strongest association with justice at work. Consistent with prior research on organizational justice and health outcomes, the researchers noted that the associations tend to be of greater magnitude for men than women, suggesting that the experience of justice may be different for the two genders. They further noted that working conditions often affect men more negatively than women, whereas the impact of family demands on health is oftentimes greater for women (Artazcoz, Borrell, Cortes, Escriba-Aguir, \& Cascant, 2005; Gimeno et al., 2010).

Similarly, De Vogli, Brunner, and Marmot (2007) also set out to investigate the relationship between unfairness and heart disease risk factors among the Whitehall II study participants and found that unfairness was associated with the metabolic syndrome and its components (positively associated with waist circumference, hypertension, triglycerides, and fasting glucose, and negatively associated with serum HDL cholesterol). However, it should be noted that unfairness was assessed with a single-item question and didn't target any of the specific justice dimensions, but was more of a global assessment of one's perception of fairness treatment.

Despite the lack of direct evidence on the relationship between organizational justice and BMI, researchers acknowledge fairness (or lack there of) 
as an emerging issue in occupational stress literature (Kelloway, Francis, \& Montgomery, 2005). If organizational justice is negatively associated with a myriad number of physical health outcomes, including high blood pressure, Type 2 diabetes, CHD, and even mortality, it follows then that BMI would also be a noteworthy outcome to measure, with its own negative relationship with organizational justice. Organizational Justice as a Moderator in the Job Stressor-Health Relationship

Despite the already established link between perceived unfairness and perceived stress, strain and anxiety (Francis \& Barling, 2005; Judge \& Colquitt, 2004; Kivimaki et al., 2005; Tepper, 2000, 2001), organizational justice has been primarily investigated as an antecedent (a "primary stressor") to strain or as a mediator between the stressor-strain relationship (Elovainio, Kivimaki, Steen, \& Vahtera, 2004; Elovainio et al., 2003; Howard \& Cordes, 2010; Taris et al., 2002), but rarely has it been looked at as a factor that would moderate or alter the magnitude of those particular relationships (Elovainio et al., 2003; Francis \& Barling, 2005; Robbins et al., 2012). And to date, there are no known studies on the moderating effects of organizational justice on the relationship between job stressors and job satisfaction, or job stressors and BMI.

Therefore, as recent organizational justice research casts a wider net to focus on employee health and well-being, this study will add to the relative deficit in our understanding that the moderating effect of justice perceptions has on the relationship between job stressors and the outcomes of job satisfaction and BMI (Elovainio et al., 2001; Robbins et al., 2012). 
Justice Theories

An important step in moving research forward is to gain an understanding of the true relationship between organizational justice and health outcomes in concert with other variables involved in employees' experience of work stress. Only then will we be able to more confidently confirm or refute the status of organizational justice as a protective factor in the prevention of job dissatisfaction as well as the development of the metabolic syndrome risk factors that lead to coronary heart disease and other deleterious health outcomes. As such the Relational Model (Lind \& Tyler, 1992) and the Effort-Reward Imbalance Model (Siegrist, 1996) are introduced as the theoretical explanations for why procedural and distributive justice in the workplace are critical to understanding employees' levels of well-being (job satisfaction) and their health outcomes (BMI).

\section{Relational Model of Authority}

Procedural justice judgments are more closely related to the perceived quality of social relationships between individuals and decision makers than having the opportunity to control the decision-making process (Tyler, 1994). The Relational Model of Authority (Lind \& Tyler, 1988; Tyler \& Lind, 1992) provides a more narrowed focus of how organizational relationships impact the behavior of employees in organizations. Specifically, the Relational Model is concerned with relational motivations, organizational outcomes dependent on interpersonal contexts, and the social bonds that exist between people and groups, group institutions, and group authorities (Tyler, 1988; Tyler \& Blader, 2003; Tyler \& Lind, 1992). Therefore, employees' perceptions that their unit, supervisor and 
organization value them are integral components to their fairness perceptions. Further, the Relational Model focuses on reactions to authority figures and suggests that neutrality, trustworthiness, and status recognition influence justice judgments (Tyler, Degoey, \& Smith, 1996). Because the premise of the Relational Model focuses on the relationship between fairness perceptions and organizational trust, which, in turn, satisfies individuals' social and psychological needs, it is well poised to explain occupational stress and health outcomes. More specifically, because justice perceptions are a function of one's understanding of their social standing with their supervisor and, in turn, their organization, organizational justice perspectives would be expected to be directly linked to subjective evaluations of one's well-being. More specifically, it is expected that individuals that perceive a lack of fairness in organizational procedures and their immediate relationships with their superiors will likely experience an increased stress reaction and a decreased sense of subjective well-being (Robbins et al., 2012).

\section{Effort-Reward Imbalance Model}

The Effort-Reward Imbalance Model (ERI; Siegrist, 1996) is closely aligned with Adams' (1965) proposition that inequity exists if the ratio between effort and reward is not proportional, and therefore, "imbalanced" as a result of a lack of reciprocity between `costs' and 'gains' (i.e., high effort/low reward conditions). At the most basic level, researchers have shown that, when high levels of effort put forth by an employee toward their work (i.e., time, money, resources) are not accompanied by equitable or appropriate rewards (i.e., salary, bonus, promotion, recognition), lowered perceptions of equity result, which can lead to increased 
physiological arousal that is consequently interpreted by the body as 'distressing' (Marvovsky, 1988).

With its focus on the distribution of rewards and resources, it's easy to see how distributive justice is closely aligned with the ERI model. Thus, when distributive justice is low, ERI is expected to be high, resulting in poorer psychological health (Robbins et al., 2012). The ERI model suggests that increased job demands, insufficient resources, lack of control, and an absence of support can all produce adverse perceptions of stress and strain, as well as a state of emotional distress, which, in turn, leads to negative health outcomes, such as poor subjective health and sickness absence (Demerouti, Bakker, Nachreiner, \& Schaufeli, 2001; Elovainio et al., 2001; Robbins et al., 2012). For example, in a large cross-sectional study on ERI and employee health, researchers found that employees reporting a mismatch between their efforts and occupational rewards showed even more pronounced risks of emotional exhaustion, psychosomatic health complaints, physical health symptoms and job dissatisfaction, compared to people reporting low job strain (de Jonge et al., 2000).

The Relational Model and the ERI Model both highlight the fact that fairness theories are appropriately suited for helping us understand why and how events and relationships in an organizational setting can initiate a cascade of psychological and physiological responses. A particular event or workplace scenario may either be viewed as positive or negative, and much of it depends on how it is interpreted by the employee, often times based on the nature of their relationship with their supervisor and organizational leaders. For instance, lowered perceptions of 
distributive justice (Greenberg, 2004, 2006) may be viewed as a fundamental contributor to an already stressful work environment for employees if they perceive they are not being benefited in proportion to their contributions or if they did not receive the promotion they feel they deserve. Lowered perceptions of procedural justice may result if organizational processes, such as selection procedures, performance appraisals or safety remediation training, are not carried out in a transparent, timely and just manner, especially if the employee feels he or she already lacks a good relationship with his supervisor.

Robbins and colleagues (2012) call for more research examining the impact of unfairness on objective measures of employee health and beg the question of whether or not fairness influences the way in which employees perceive their health, or whether there is an actual tangible impact on their health. Robbins et al.'s (2012) meta-analysis aptly points out that to date there has been little consistency in the way in which the relationship between fairness perceptions and health has been studied. Therefore, bearing the aforementioned fairness theories in mind, it is expected that adverse occupational stress and health outcomes will emerge as responses to lowered perceptions of justice. If conceptualized as an environmental condition, several managerial implications emerge; namely that organizational leaders are in the position to increase their awareness of the problematic work environment climate, and, more importantly, to take steps to moderate the impact of negative events and weakened relationships. 


\section{Chapter V: Outcome Variables}

The purpose of this chapter is to briefly discuss the outcome variables of this dissertation - job satisfaction and body mass index (BMI). As such, I provide definitions for both job satisfaction and BMI, discuss their known antecedents, outcomes, and control variables, and provide a brief explanation of how they are measured in research.

\section{Definition of Job Satisfaction}

Locke (1976) described job satisfaction as "a pleasurable or positive emotional state resulting from the appraisal of one's job or job experiences" (p. 1304). Locke's definition assumes that job satisfaction is the interplay of cognition (appraisal) and affect (emotional state), or thoughts and feelings (Judge et al., 2002). Cranny, Smith, and Stone (1992) also stated that "although definitions of the construct vary somewhat from one work to another, there appears to be general consensus that job satisfaction is an affective reaction to a job that results from the incumbent's comparison of actual outcomes with those that are desired (expected, deserved, and so on)" (p. 1). Hulin and Judge (2003) also defined job satisfaction as the multidimensional psychological responses to one's job, which have cognitive (evaluative), affective (or emotional), and behavioral components. However, the researchers also noted that the I/O field has departed from the original tripartite definition as research has tended to focus on the judgment-based, cognitive evaluations of jobs, on characteristics or features of jobs, and generally ignored the affective antecedents of evaluations of jobs and episodic events that happen on jobs. 
The cognitive evaluations, in turn, have largely overshadowed the systematic measurement of affect and emotion as causes, components, or consequences of job satisfaction (Weiss \& Brief, 2001). According to Judge and colleagues (2002), most scholars recognize that job satisfaction comprises of various facets, with the most common being: pay, promotions, coworkers, supervision, and the work itself (Smith, Kendall, \& Hulin, 1969). Locke (1976) later added recognition, working conditions, and company and management.

\section{Brief Historical Background on Job Satisfaction}

The annual review by Brief and Weiss (2002) notes that the study of affect at work (or job satisfaction) emerged in the United States in the 1930s as a perceived need to understand workers' feelings in the minds of those who managed them. In fact, Hoppock's (1935) landmark book, which coincided with the Hawthorne Studies (Roethlisberger \& Dickson, 1939), may have unknowingly marked the beginning of the human relations movement (Judge et al., 2002) with its opening remark stating, 'Whether or not one finds his employment sufficiently satisfactory to continue in it... is a matter of the first importance to employer and employee' (p. 5).

Now-classic studies from this time period that focused on job satisfaction include Fisher and Hanna's (1931) The Dissatisfied Worker, Kornhauser and Sharp's (1932) “Employee attitudes; suggestions from a study in a factory," Hersey’s (1932) Workers' Emotions in Shop and Home: A Study of Individual Workers from the Psychological and Physiological Standpoint, Hoppock's (1935) Job Satisfaction, and Roethlisberger and Dickson's (1939) Management and the Worker. Relying on the findings of case studies, Fisher and Hanna (1931) characterized job dissatisfaction 
as the product of "nonadjustive emotional tendencies" whose associated unrest is misattributed by workers to their job situations. Hoppock (1935), using surveys and interviews of teachers in a Pennsylvania community, found that elements of the work environment (e.g., supervision), family expectations, and "emotional maladjustment," all influence employees' job satisfaction. Moreover, data from AT\&T's Western Electric Hawthorne plant employees led Roethlisberger and Dickson (1939) to conclude that the workplace's social organization and the individual's interaction with the organization, more than individual differences, determine worker adjustment/happiness.

Antecedents of Job Satisfaction

Judge and colleagues (2002) noted that the antecedents of job satisfaction may loosely fall into one of three categories: 1) situational/ environmental, 2) dispositional/personality, and 3) the interplay of the two.

Situational/Environmental Antecedents. The Job Characteristics Model (JCM; Hackman and Oldham, 1976) argues that jobs that contain intrinsically motivating characteristics will lead to higher levels of job satisfaction. The five core job characteristics discussed in the JCM consist of task identity (degree to which one can see one's work from start to finish), task significance (degree to which one's work is seen as important and significant), skill variety (extent to which job allows employees to do different tasks), autonomy (degree to which employees have control and discretion for how to conduct their job), and feedback (degree to which the work itself provides feedback for how the employee is performing the job). These core characteristics are said to lead to three critical psychological states - 
experienced meaningfulness of the work, responsibility for outcomes, and knowledge of results, all of which lead to outcomes. According to the Hackman and Oldham (1976), the relationship between intrinsic job characteristics is dependent on employee's Growth Need Strength (GNS), which encapsulates one's desire for personal development with regards to work. Employees high on GNS want their jobs to contribute to their personal development, and they derive satisfaction from performing challenging and personally rewarding activities. It has been well established in the literature that autonomy in the work environment has an overall positive effect on satisfaction (e.g., Hackman and Lawler, 1971; Humphrey, Nahrgang, \& Morgeson, 2007).

Dispositional/Personality Antecedents. The impact of individual differences on job satisfaction has long been recognized since the early works of Hoppock (1935) who found that satisfied workers were better adjusted emotionally compared to dissatisfied workers. Review of the literature indicates that studies fall into two broad categories: indirect studies, which seek to demonstrate a dispositional basis to job satisfaction by inference, and direct studies, which relate a direct measure of a construct purported to assess a personality trait to job satisfaction.

Through a process of logical deduction, several researchers have indirectly inferred a dispositional source of job satisfaction after observing that measures of job satisfaction were relatively stable over five-year periods, even after individuals changed employers and occupation (Staw \& Ross, 1985). Studies on monozygotic twins reared apart from early childhood also found significant similarity in job satisfaction levels among 34 pairs of identical twins (Arvey, Bouchard, Segal, \& 
Abraham, 1989).

With regards to direct dispositional studies, several studies have found that both positive and negative affectivity (PA = high energy, pleasurable engagement, and enthusiasm; NA = distress, unpleasurable engagement and nervousness) are related to job satisfaction (Thorensen \& Judge, 1997). Additionally, meta-analytic results on 169 independent correlations found that core self-evaluations, or the fundamental premise that individuals hold about themselves and their functioning in the world, correlated .37 with job satisfaction (Judge \& Bono, 2001). Judge and colleagues (2002) have also observed a positive link between individualism and job satisfaction when comparing samples of workers across countries, while studies within a country found collectivism to have a positive relationship with job satisfaction. With regards to the Big Five personality factors, meta-analytic evidence suggests that neuroticism, conscientiousness and extraversion may all be useful predictors of job satisfaction (Judge et al., 2002).

Interactive Antecedents (Situation and Personality). Interactive theories of job satisfaction are those that consider both the person and the situation. According to the Cornell Model (Hulin, Roznowski, and Hachiya, 1985), job satisfaction is a function of the balance between work role contributions (e.g., skills, time, effort, training) and work role outcomes (e.g., salary, fringe benefits, status, working conditions, and intrinsic outcomes). All else being equal, the model proposes that job satisfaction will be higher if more work role outcomes are received relative to the contributions invested.

Locke's (1976) Value-Percept Theory argues that employees' values are what 
determine what is satisfying to them on the job. The model expresses job satisfaction as the value content of what one wants, minus the perceived amount of the value provided by the job, multiplied by the importance of the value to the individual. A central tenet of the model is that what one desires is expected to be highly correlated with what one deems important, with pay being an obvious example (it would not be highly desired if it were not also important).

In using a 'fit' framework, Verquer, Beehr, and Wagner (2003) metaanalytically examined the utility of PO fit in predicting job satisfaction and found a moderate effect size of .25. A meta-analysis by Kristof-Brown and colleagues (2005) summarized 47 studies $(\mathrm{N}=12,960)$ and found an average correlation of .44 and .45 , respectively, between person-job and person-organization fit with job satisfaction. Control Variables of Job Satisfaction

Several variables have been investigated as controls for job satisfaction. For example, demographic variables such as age, gender, tenure, performance, number of hours worked, psychological well-being, and composite job satisfaction have all demonstrated high correlations and potential relationships with job satisfaction, and thus should be important factors to consider when conducting research on job satisfaction (Berkman, 1971; Blum \& Naylor, 1968; Brush, Mock, \& Pooyan, 1987; Cranny, Smith, \& Stone, 1992; Spector, 1987). Outcomes of Job Satisfaction

Given the centrality of job satisfaction to I/O Psychology, it is not surprising that it has been found to be an important antecedent to several beneficial personal and organizational outcomes. A meta-analysis by Tait, Padgett and Baldwin (1989) 
revealed a moderately strong correlation (.44) between job satisfaction and life satisfaction. This relationship is expected to be reciprocal, such that a happy nonwork life would spill over onto one's job experiences and evaluations, just as a stressful work experience is expected to spillover onto one's life outside of work (Judge et al., 2002).

With regards to organizational outcomes, research has shown strong links between job satisfaction and motivation, intentions to leave the organization, absenteeism, turnover, lateness, and both objective and subjective performance criteria (Kinicki, McKee-Ryan, Schriesheim, \& Carson, 2002), as well as attendance at work (Scott \& Taylor, 1985), drug abuse, decisions to retire (Hanish \& Hulin, 1990, 1991), psychological withdrawal behaviors (Roznowski, Miller, \& Rosse, 1992), prosocial and organizational citizenship behaviors (Organ \& Ryan, 1995), and unionization activity (Hamner \& Smith, 1978; Zalesny, 1985). Measures of Job Satisfaction

The Job Descriptive Index (JDI; Smith et al., 1969; Cranny et al., 1992) is the most widely used measure of job satisfaction. It assesses five facets of job satisfaction (work itself, pay, promotional opportunities and policies, supervision, and coworkers) by asking participants to describe their jobs in terms of the presence or absence of 72 characteristics. The five scales have been used extensively in research as both antecedents and outcomes of job attitudes (Judge et al., 2002). Other well-received standardized instruments in the field include the Job Diagnostic Survey (JDS; Hackman \& Oldham, 1976), the Minnesota Satisfaction Questionnaire (MNSQ; Dawes, Dohm, \& Lofquist, 1967), and the Index of 
Organizational Reactions (IOR; Dunhan \& Smith, 1979). Aside from the issue of whether or not to measure job satisfaction via facets or as an overall measure (the sum of the facets), researchers have also long argued the reliability of a single-item measure (Judge et al., 2002). Although not ideal, and certainly not as high as most multi-item measures, Wanous, Reichers, and Hudy (1997) found the reliability of single-item measures of job satisfaction to be an acceptable .67.

\section{Definition of Body Mass Index}

Body mass index (BMI), a measurement of weight and obesity, is one of the primary risk indicators of CHD, hypertension, stroke, Type 2 diabetes, and certain cancers (Kouvonen et al., 2005). The terms overweight and obesity refer to body weight that is greater than what is considered healthy for a certain height. The etiology of obesity is not well understood, but it is related to an interaction between biological and environmental factors and said to be the result of family history and genetics, metabolism (how the body changes food and oxygen into energy), as well as one's habits (Nyberg et al., 2012). Not only are genes important in determining susceptibility to gain weight, but research has also shown that key behavioral factors are an increased consumption of energy-dense foods high in saturated fats and sugars and reduced physical activity (Kouvonen et al., 2005). Antecedents of BMI

Despite mixed results, high demands, low control, obesity and BMI at baseline, lack of physical activity, lack of sleep, and alcohol consumption have all been investigated as determinants of BMI (Lalluka et al, 2005; Kivimaki et al., 2005; Nyberg et al., 2012). In a recent study on over 160,000 adults from 13 cohort 
studies, Nyberg and colleagues (2012) also determined that onset of job strain was associated with a U-shaped change in BMI (individuals with lean BMI lost weight whereas individuals with high BMI gained weight). Job control and social stressors (described by Dormann \& Zapf (2002) as social animosities, conflicts with coworkers and supervisors, unfair behavior, and a negative group climate) have also been identified as statistically significant predictors of follow-up BMI, while effortreward imbalance was marginally significant (Berset et al., 2011). Control Variables of BMI

Control variables of BMI include demographic variables such as age, gender, baseline BMI and obesity, cholesterol, hypertension, and alcohol consumption (Kivimaki et al., 2005, 2006). Nyberg and colleagues (2012) also noted that socioeconomic status (SES; high, intermediate, and low) and smoking status (current smoker versus non-smoker) were also noteworthy variables that should be controlled for in research. SES is oftentimes investigated as a composite of occupation, income and education. Some have suggested that this association exists because environmental conditions associated with low levels of education limit one's access to and knowledge of nutritious food choices and safe means to exercise, it may bring on metabolic dysregulation, and trigger the consumption of high caloric foods. Environments associated with low SES also increase the expression of genetic vulnerabilities to health problems - including obesity (Johnson, Kyvik, Skytthe, Deary, \& Sorensen, 2011). 
Outcomes of BMI

As mentioned previously, being overweight or obese puts individuals at higher risk for many health problems because the more body fat that individuals have, the more likely they are to develop CHD, hypertension, stroke, high blood pressure, Type 2 diabetes, gallstones, sleep apnea, reproductive problems, and certain cancers (Berset et al., 2011; CDC, 2012). BMI is also an important driver of direct medical costs, and indirect costs, such as absenteeism or workplace injuries (Schmier, Jones, \& Halpern, 2006).

Measurement of BMI

BMI is calculated as weight in kilograms divided by height in meters squared. In adults, BMI is separated into quintiles: the lean quintile is comprised of BMI $<23 \mathrm{~kg} / \mathrm{m}^{2}$, the middle quintile is BMI in the range of $23-27 \mathrm{~kg} / \mathrm{m}^{2}$, and the high quintile is BMI $>27 \mathrm{~kg} / \mathrm{m}^{2}$. BMI greater than or equal to $30 \mathrm{~kg} / \mathrm{m}^{2}$ equates to obesity. Obesity can be further broken down into class I (BMI 30 to $<35 \mathrm{~kg} \mathrm{~m}^{2}$ ) and class II/III (BMI > $35 \mathrm{~kg} \mathrm{~m}^{2}$ ). In children, obesity is defined as a BMI greater than or equal to the age- and sex-specific $95^{\text {th }}$ percentiles of the 2000 CDC growth charts (CDC, 2012). 


\section{Chapter VI: Hypotheses}

The purpose of this chapter is to outline the hypotheses for this dissertation. Due to the number of variables of interest in this study, I have separated the hypotheses by outcome, such that all hypotheses related to job satisfaction are addressed first, followed by hypotheses related to BMI. On the basis of the CFA conducted on the job stressor variables (see Results section), all hypotheses for job control are spelled out separately for skill discretion and decision authority.

The first set of hypotheses involves predictions regarding the main effects of job stressors (i.e., job demands, skill discretion, and decision authority) on job satisfaction, then the main effects of organizational justice (distributive and procedural justice) on job satisfaction, followed by the moderating effect of organizational justice on the relationship between job stressors and job satisfaction. The second set of hypotheses involves predictions regarding the main effects of job stressors on BMI, then the main effects of organizational justice on BMI, followed by the moderating effect of organizational justice on the relationship between job stressors and BMI. The third set of hypotheses states predictions regarding the relationship between job stressors and the outcomes of job satisfaction and BMI as partially mediated by exercise. The last set of hypotheses states the mediated moderation predictions for the effect of the interaction of organizational justice and job stressors on BMI and job satisfaction as mediated through exercise. Figure 1

presents a model that illustrates all of the proposed hypotheses in this study. Table 1 lists all of the study hypotheses and related results. 
The Main Effects of Job Stressors on Job Satisfaction

Job satisfaction has long been considered a central and enduring outcome of interest when it comes to individual-level organizational research (Harrison et al., 2006; Hulin \& Judge, 2003). Employee health models, such as the JD-C (Karasek, 1979), posit that increased job demands produce greater perceived stress and strain, which in turn leads to more negative health outcomes, with job satisfaction being one of the most studied subjective well-being outcomes of interest (Bellavia \& Frone, 2005; Fox et al., 1993; Parkes, Menham, \& Rabenau, 1994; Spector, Chen, \& O'Connell, 2000; Stansfeld, Bosma, Hemingway, \& Marmot, 1998; Wolpin, Burker, \& Greenglass, 1991; van der Doef \& Maes, 1999). Further, a recent review of the relevant research on occupational strain and employee health suggests that there is good evidence for the main effects of job demands on job satisfaction (Beehr \& Glazer, 2005; de Lange et al., 2003; Quick et al., 1997).

With regards to job control, numerous studies have also demonstrated clear associations between poor job control and low job satisfaction (Elovainio \& Kivimaki, 1996; Hackman \& Oldham, 1975). Elovainio and colleagues (2000) found that job control clearly accounted for most of the variability in job satisfaction at both the organizational and individual level among their nurse population. When considering both demands and control, Theorell (2003) found that people in jobs with high demands and low control experience more job dissatisfaction, amongst other negative health outcomes. Similarly, van der Doef and Maes (1999) found that working in high stress jobs was associated with lower job satisfaction, more 
burnout, lower general psychological well-being, as well as lower job-related psychological well-being.

Therefore, on the basis of the JD-C Model (Karasek, 1979) and in support of previous research on the impact of occupational stressors on job satisfaction, I hypothesize that the individual components of job strain (job demands, skill discretion, and decision authority) will each have a direct impact on employees' levels of job satisfaction. More specifically, I propose that job demands will have a negative relationship with job satisfaction such that as job demands increase, job satisfaction will decrease. Additionally, I propose that the individual components of job control (skill discretion and decision authority) will each have a positive relationship with job satisfaction, such that as levels of skill discretion and decision authority increase so will one's level of job satisfaction.

H1a. Job demands will be negatively related to job satisfaction.

H1b. Skill discretion will be positively related to job satisfaction.

H1c. Decision authority will be positively related to job satisfaction. The Main Effects of Organizational Justice on Job Satisfaction

Organizational justice research has consistently demonstrated that perceptions of fairness can impact employees' attitudes and behaviors at work (Colquitt et al., 2001; Judge \& Colquitt, 2004). DeConinck and Stilwell (2004) found a strong association between individuals' perceptions of fairness in the workplace and their levels of satisfaction with their job, supervisor, pay and coworkers. Tepper (2001) found that employees who held less favorable justice perceptions experienced greater psychological distress. Additionally, recent meta-analytic works 
have also shown that both procedural and distributive justice are linked to employee well-being and psychosocial outcomes, such as job satisfaction (Colquitt, 2001; Colquitt et al., 2001; Colquitt \& Greenberg, 2003; Robbins et al., 2012).

Moreover, in response to the notion that justice may be a universally desired feature in the workplace, research on the global effects of justice on non-Western cultures has found that procedural and distributive justice had significant and equally strong effects on job satisfaction across employee samples from Australia, Columbia, India, Jordan, and Saudi Arabia, in addition to the United States (Pillai et al., 1999; Shao, Rupp, Skarlicki, \& Jones, 2013).

All of the previously mentioned research provide ample support for the view that perceived fairness is an imperative determinant of one's subjective well-being, especially within one's workplace environment whereby a majority of one's time is spent in direct contact their with supervisors. That relationship with the supervisor, interpreted as fair or unfair treatment, is in turn responsible for a whole host of both positive and negative responses (Folger \& Cropanzano, 2001; Lind, 2001; Siegrist, 1996). Therefore, in support of previous organizational justice research and the underpinnings of the Relational Model and the ERI model, I propose that the organizational justice dimensions will have a direct impact on individuals' levels of job satisfaction. More specifically, I propose that both distributive justice and procedural justice will be positively related to job satisfaction such that as employees' perceptions of distributive and procedural justice increase, so will their levels of job satisfaction.

H2a. Distributive justice will be positively related to job satisfaction. 
H2b. Procedural justice will be positively related to job satisfaction.

The Moderating Effects of Organizational Justice on the Relationship Between Job Stressors and Job Satisfaction

A plethora of studies have investigated injustice as a workplace stressor itself (Kivimaki et al., 2008; Taris et al., 2002), as well as a mediating mechanism between a stressor and various health outcomes (Brotheridge, 2003; Elovainio et al., 2001; Elovainio et al., 2004). However, very few to date have examined the effects of justice as a moderator (or a "buffer") in the occupational stress-job satisfaction relationship. The few that have, investigated health outcomes that are psychosocial in nature (i.e., mental health - depression, distress, anxiety), and the overall results of the moderated effects were found to be non-significant or mixed (Elovainio et al., 2001; Francis \& Barling, 2005).

In examining whether or not perceptions of justice are a relevant moderator in the job stressor - job satisfaction relationship, it is important to consider employees' established bonds with their organization and their supervisors. For example, Tepper (2001) explored the interaction of both justice dimensions and found that the relationships between procedural justice and psychological distress were stronger when distributive justice was lower (but not vice versa). According to Tepper (2001), under conditions of low distributive justice, perceptions of procedural unfairness prompt employees to regard threats as "stressful" (which produces higher levels of psychological distress), while perceptions of procedural fairness engender perceptions that threats are "benign" (which produces lower levels of psychological distress). Thus, to the extent that organizational injustices 
(caused by supervisors' actions or organizational procedures) are viewed as stressors or threats to employees that are already experiencing high stress at work, the likely outcome is increased levels of psychological distress and lowered levels of job satisfaction. This relationship may be extremely salient in an internal promotional selection process in that an unfavorable outcome (interpreted as low distributive justice) has the potential to be viewed as benign or detrimental depending on the interplay between the stress of the overall experience and the employees' feelings of procedural (in)justice regarding the selection process (trust in their superiors, transparency of the internal organizational processes, etc.) (Ford Truxillo, \& Bauer, 2009).

According to Weiss and Cropanzano (1996), negative events are thought to produce negative emotion responses as well as a cognitive appraisal, especially if the event triggers a negative emotional response because the employee feels some harm has been done. Research by McColl-Kennedy and Sparks (2003) in the service industry demonstrated that service providers can greatly influence customer feelings and can, through their specific actions, modify customer emotions of anger and dissatisfaction. Successfully modifying customers' feelings and lowering their levels of anger produced positive emotions for the service providers, while unsuccessful modifications of customers' feelings of dissatisfaction brought about a negative response. On the basis of the ERI Model, one would anticipate that feelings of dissatisfaction may arise if employees feel as though they have been poorly compensated for high levels of effort that they have invested into the job, team, customer, or organization. A high imbalance of effort (inputs) and rewards 
(outputs) have been shown to result in mental disorders, decreased levels of selfesteems, anger, anxiety, and depression, all suggestive of a link between perceived unfairness and negative psychological conditions (Robbins et al., 2012; Siegrist 1996). Additionally, as with the findings of Karasek (1979) on the moderating effects of supervisor support on high-strain jobs, there is an expectation that employees will report increased levels of job satisfaction when their supervisors appear to be more fair in their handling of organizational procedures or in their allocation of rewards. The opposite is also true - employees that view their supervisors to be less fair and transparent in their procedural and distributive behaviors may experience increased feelings of injustice, which are inflammatory by nature, and thus, expected to further exacerbate the relationship between occupational stressors and job dissatisfaction.

Therefore, on the basis of the Relational Model, the ERI Model, and the JD-C, I propose that organizational justice will buffer the negative effects of job stressors on job satisfaction. While I don't anticipate that organizational justice would necessarily have a substantial impact of low job demands or high job control on job satisfaction, I do anticipate that higher perceptions of distributive and procedural justice will lower the impact that job stressors have on employees' levels of job satisfaction. Under conditions of high job demands, lowered perceptions of procedural (distributive) justice would prompt employees to feel even more threatened or a heightened sense of resource loss, which would result in lowered levels of job satisfaction. Similarly, under times of high demands, employees with high perceptions of procedural (distributive) justice are expected to experience less 
threatened and a heightened sense of resource gain (or simply preservation), which would translate to higher levels of job satisfaction.

H3a/b. Organizational justice will moderate the relationship between job demands and job satisfaction, such that higher perceptions of distributive (procedural) justice will mitigate the negative effects of high job demands on job satisfaction.

H4a/b. Organizational justice will moderate the relationship between skill discretion and job satisfaction, such that higher perceptions of distributive (procedural) justice will mitigate the effects of lower skill discretion on job satisfaction.

H5a/b. Organizational justice will moderate the relationship between decision authority and job satisfaction, such that higher perceptions of distributive (procedural) justice will mitigate the effects of lower decision authority on job satisfaction.

The Main Effects of Job Stressors on Body Mass Index

The premise of the JD-C model has also been investigated to determine a connection between job stressors and physical health outcomes, such as CHD, risk factors associated with CHD, negative pregnancy outcomes, gastrointestinal disorders, and increased psychosomatic complaints (Belkic, Landsbergis, Schnall, \& Baker, 2004; Schnall et al., 1994; Theorell, 2003; van der Doef \& Maes, 1998). It has been demonstrated that the demands stemming from one's work role can have a negative impact on the health of an employee (Bellavia \& Frone, 2005; van der Doef \& Maes, 1999). Despite this already established relationship between job stressors 
and health outcomes, a gap exists in the literature regarding whether or not job stressors are directly related to BMI (Nyberg et al., 2012; Robbins et al., 2012).

Kivimaki and colleagues (2006) noted that despite the common understanding that work stress and high BMI are both risk factors for CHD and other chronic and fatal health conditions, the extent to which they are connected remains unclear, lending researchers to interpret the inconsistent findings as an indication of the absence of an association. While some studies have clearly found a connection between high demands, low control, and increased BMI (Hellerstedt \& Jeffery, 1997; Wamala et al., 1997; Niedhammer et al., 1998; Lallukka et al., 2005), other studies have reported no associations between these psychosocial characteristics of the work environment and BMI (Brisson et al., 2000; Reed et al., 1989; Jonsson et al., 1999; Landsbergis et al., 1998). Thus, on the basis of the JD-C Model and previous research that has found a relationship between job stressors and BMI, I also propose that job stressors will have a direct effect on BMI; namely that as demands increase, so will one's BMI, and that the lowering of one's skill discretion and decision authority at work will also be associated with increases in one's BMI.

H6a. Job demands will be positively related to BMI.

H6b. Skill discretion will be negatively related to BMI.

H6c. Decision authority will be negatively related to BMI. 
The Main Effects of Organizational Justice on Body Mass Index

Judge and Colquitt (2004) argue that lowered perceptions of fairness and unfair treatment can potentially have a "visceral and even physiological effect" on employees, thereby disrupting their work attitudes and behaviors, as well as overall health. Evidence of the impact of injustice on physical health, such as sleeping problems, self-rated health status, incidence of psychiatric morbidity and sick leave (Elovainio et al., 2001; Elovainio et al., 2002; Elovainio et al., 2003; Elovainio et al., 2004, Kivimaki et al., 2003a; Kivimaki et al., 2003b) has begun to emerge in the literature, though consistent objective results are sporadic, indirect or altogether lacking (Robbins et al., 2012). And despite the cumulative evidence supporting the existence of a link from unfair treatment to experiences of strain and various health problems following prolonged stress exposure (Elovainio et al., 2001, 2002;

Kivimaki et al., 2003a; Kivimaki et al., 2003b), empirical evidence on the direct relationship between organizational justice and BMI is also sorely lacking.

Considering that elevated BMI and obesity appears to be a gateway to several other chronic and fatal conditions (Bosma et al., 1997; Kivimaki, et al., 1997), it is understandable that researchers are attempting to further expand its determinants. Accordingly, justice has gained a position in the recent empirical health studies. For example, Gimeno and colleagues (2010) found that men who experienced a high level of workplace justice had a $25 \%$ lower risk of incident metabolic syndrome than employees with a low level of justice, with large waist circumference having the strongest association with justice at work. Additionally, De Vogli, Brunner, and 
Marmot (2007) also found that unfairness was associated with metabolic syndrome components (one of which was higher waist circumference).

A recent meta-analysis by Robbins and colleagues (2012) found that health may suffer when individuals experience unfairness in the workplace, as evidenced by somewhat higher incidence of physical health problems $(\rho=.10)$. Physical health in their study was operationalized as BMI, cholesterol, blood serum, heart rate, hypertension, cancer, myocardial infractions, cardiovascular reactivity, illnesses, physical health complaints, physical symptoms, and disease. Specifically, metaanalytic results by Robbins and colleagues (2012) show that procedural justice and distributive justice are significantly predictive of objective health problems $(\rho=.25$ and $\rho=.15$, respectively), one of which was BMI.

Thus, on the basis of the Relational Model and the ERI Model, as well as in support of previous research on the relationship between justice and BMI, I propose that organizational justice (both procedural and distributive justice) will have a direct impact on employee's BMI readings. Specifically, I propose that higher distributive and procedural justice perceptions will be associated with lower BMI.

H7a. Distributive justice will be negatively related to BMI.

H7b. Procedural justice will be negatively related to BMI.

The Moderating Effect of Organizational Justice on the Relationship Between Job Stressors and Body Mass Index

Researchers have long been interested in the impact that fairness, or lack thereof, has on individuals, especially in the workplace environment. Considering that employees are highly dependent on their supervisors for resources, rewards, 
and information, their relationships with their supervisors are critically important to their standing in the organization and their overall levels of health and wellbeing. Several studies provide support for the fact that poor and abusive leadership consistently has a negative impact on employee psychological health - namely increased stress (Offermann \& Hellmann, 1996), psychological distress (Richman, Flaherty, Rospenda, \& Chistensen, 1992), low levels of job and life satisfaction, lower levels of affective commitment, increased work-family conflict, and psychological distress (Tepper, 2000), as well as psychosomatic symptoms such as anxiety and depression (Hoel, Rayner, \& Cooper, 1999).

Justice research has recently broadened its focus to include employee objective health (Kivimaki et al., 2008; Robbins et al., 2012). As mentioned previously, Robbins and colleagues (2012) conducted a meta-analysis on perceived unfairness and employee health and found that individuals who experienced unfairness had higher incidences of physical health problems (i.e., hypertension, high cholesterol, high body mass index, physical health complaints). Gimeno and colleagues have demonstrated that high levels of justice are related to reduced work stress, and thus related to a lowered risk of developing metabolic syndrome (one of which is BMI). Other research has also looked at the impact of the stress of poor supervision on systolic and diastolic blood pressure (Wager, Fieldman, \& Hussey, 2003), and cardiovascular disease (Kivimaki et al., 2003).

On the basis of the JD-C model, Kelloway and colleagues (2005) posit that abusive and passive leadership are exemplars of poor leadership that contribute to increased employee stress in that they are the root cause of workplace stressors 
(Kelloway et al., 2005). Moreover, meta-analytic findings by Viswesvaran, Sanchez, and Fisher (1999) show that social support has both a direct and an indirect effect on the stressor-strain relationship. These findings are suggestive of the fact that leaders' support (or lack there of) will also have both a direct and indirect effect on their followers. In using House's (1981) classification of support, leaders have the responsibility for providing instrumental support (task specific help), emotional support (empathy, affect, and comfort), informational support (awareness, advice, and directives), and appraisal support (feedback, suggestions, and encouragement). Kelloway and colleagues (2005) argue that poor leaders not only fail to provide support to buffer stressful work conditions, but they also neglect to provide their followers the bare amount of support that followers desire to function without stress. Also, on the basis of the Ego Depletion Model, if acts of fairness by a supervisor (providing employees accurate and timely information, establishing clear and transparent processes, allocating resources, tools, and materials in an fair and equitable manner, etc.) are perceived by employees as resource-building, or even simply as not a drain on their resources, then it would follow that higher perceptions of justice would also act as a buffer in the stressor-strain relationship as well.

The heightened focus on the relationship between organizational justice and employee physical health is highly warranted, not only to offset disease and premature morbidity (Bosma et al., 1997; Kivimaki, et al., 1997), but also because fairness is a changeable aspect of the workplace, making it a prime target for interventions that focus specifically on job stress, employee health, and supervisor 
training (Robbins et al., 2012). If the source of stressors is the work environment, supervisors are in the prime position to have the largest positive impact on these stressors through their various ways of providing support and acting in a fair and equitable manner. However, information, rewards, interpersonal treatment, support and fairness provided by a supervisor may not be beneficial, and may in fact be detrimental, to the employee if the supervisor is perceived by the employee to be the very source of the stress (Kelloway et al., 2005).

Therefore, on the basis of the Relational Model and the Ego Depletion Model, I propose that employees' perceptions of organizational justice will buffer the negative effects of job stressors on BMI. Specifically, I anticipate that higher perceptions of distributive and procedural justice perceptions will lower the impact that high stress jobs have on employees' BMI readings.

$\mathbf{H 8 a} / \mathbf{b}$. Organizational justice will moderate the relationship between job demands and BMI, such that higher perceptions of distributive (procedural) justice will mitigate the negative effects of high job demands on BMI. H9a/b. Organizational justice will moderate the relationship between skill discretion and BMI, such that higher perceptions of distributive (procedural) justice will mitigate the effects of lower skill discretion on BMI.

H10a/b. Organizational justice will moderate the relationship between decision authority and BMI, such that higher perceptions of distributive (procedural) justice will mitigate the effects of lower decision authority on BMI. 
Exercise as a Partial Mediator in the Job Stressor-BMI Relationship

It has been demonstrated that the demands stemming from one's work role can have a negative impact on the health of an employee (Bellavia \& Frone, 2005; van der Doef \& Maes, 1999). Despite the plethora of studies on the association between job stressors and objective physical health, there is a general lack of empirical evidence on the direct relationship between job stressors and BMI (Nyberg et al., 2012). Rather than interpreting the inconsistent findings as an indication of the absence of an association, some researchers have posited that this relationship may be better understood via a logical pathway connecting the two, such as the health behavior of exercise (Pratt, 1971; Robbins et al., 2012).

Not surprisingly, studies that have investigated both BMI and exercise have yielded mixed results. In a longitudinal study on over 15,000 men and women, Kujala and colleagues (1998) found that both "occasional exercisers" and "conditioning exercisers" had reduced risks of mortality compared with those who were "sedentary", pointing to the beneficial effect of physical activity after controlling for other predictors of mortality (age and sex) (Kujala, Kaprio, Sarna, \& Koskenvuo, 1998). A recent meta-analysis by Conn and colleagues (2009) on workplace physical activity interventions found small but positive effect sizes for BMI, indicating better scores following interventions among the treatment subjects. Additionally, several meta-analyses have found significant improvements in stress reactivity after some form of physical activity (Alderman et al., 2004; Ramirez \& Wipfli, 2013). Not only are exercise-induced techniques applicable to the general population for reductions in stress reactivity, but over time such reductions have 
the potential to reduce the burden of serious medical conditions associated with stress, such as CHD, CHD risk factors, Type 2 diabetes, and immune system dysfunction.

Empirical studies have suggested that the costly toll of numerous health problems can actually be reduced through a healthy diet, regular exercise, proper sleep and rest habits, restraint from smoking, alcohol consumption, or substance abuse, and reduced risk-taking behavior (Matarazzo, 1982; Pratt, 1971; Williams \& Wechsler, 1972). Research has also shown that stressful situations with many demands on employees' time and limited resources leave employees feeling a lack of control and with the overwhelming perception that maintaining good health practices is a challenge (Langlie, 1977). This is a common occurrence in high-stress incidences that induce negative responses such as aggravated signs of disease, increases in alcohol consumption, a tendency to smoke, or in the consumption of energy-dense foods high in saturated fats and sugars, and physical inactivity (Horowitz et al., 1979; Schachter, Silverstein, Kozlowski, Herman, \& Liebling, 1977). Consist with the Ego Depletion Model (EDM), it follows that a high stress job would drain an employee of his available resources (mental and physical) that are necessary for one to find the time and/or energy to engage in physical activity, which has been shown to influence bodily weight and BMI. Thus, on the basis of the EDM and in support of empirical evidence that key behavioral factors such as lack of physical activity leads to obesity (Kouvonen et al., 2005), I propose that exercise will act as a partial mediator in the job stressor-BMI relationship. 
H11a. Exercise will partially mediate the relationship between job demands and BMI.

H11b. Exercise will partially mediate the relationship between skill discretion and BMI.

H11c. Exercise will partially mediate the relationship between decision authority and BMI.

Exercise as a Partial Mediator in Job Stressor-Job Satisfaction Relationship

As mentioned previously, the job stress literature has a long history of demonstrating that work stressors are related to job satisfaction (Bellavia \& Frone, 2005; Fox et al., 1993; Parkes et al., 1994; Spector et al., 2000; Stansfeld et al., 1998; Wolpin et al., 1991), with evidence to suggest that both high job demands (Beehr \& Glazer, 2005; de Lange et al., 2003; Quick et al., 1997) and low job control (Elovainio \& Kivimaki, 1996; Elovainio et al., 2000; Hackman \& Oldham, 1975; Theorell, 2003; van der Doef \& Maes, 1999) have a clear association with lower job satisfaction, more burnout, lower general psychological well-being, as well as lower job-related psychological well-being. Physical activity has been documented as being beneficial for offsetting CHD risk and disease prevention, as well as promoting psychological well-being, namely warding off depression, lowering anxiety, and enhancing life and job satisfaction (Rethorst et al., 2009). For example, Kirkcaldy and colleagues (1994) conducted a large-scale study of leisure habits and satisfaction on 533 senior police officers (aged 28-58 years) from the UK and found that exercisers had a higher level of job satisfaction and better physical and mental health than non-exercisers (Kirkcaldy, Cooper, Shepard, \& Brown, 1994). Similarly, Daley and Parfitt (1996) 
examined differences in mood states, physical well-being, job satisfaction, and absenteeism in members, non-members, and individuals on the waiting list for a British corporate fitness club. Results from the study suggest that club members have better psychological mood states and physical well-being than non-members, and that members were more satisfied with their jobs and took fewer sick days than non-members. In a relatively recent meta-analysis of workplace physical activity interventions, Conn and colleagues (2009) found that job satisfaction was significantly greater among treatment subjects than controls following interventions in the two-group pre-post effect analysis (effect size $=.20$ ).

As results have shown that numerous health and psychological problems can be reduced through health behaviors, such as regular exercise (Pratt, 1971; Williams \& Wechsler, 1972), and that maintaining good health practices is a challenge (Langlie, 1977) in the face of stressful situations with many demands on employees' time and limited resources, it follows that a high stress job would drain an employee of his available resources (mental and physical) necessary for him to engage in physical activity, which would result in lower levels of job satisfaction (Conn et al., 2009; Daley \& Parfitt, 1996; Kirkcaldy et al., 1994), especially if the employee views his job as the culprit that is keeping him from engaging in other activities other than work. Similar to the effects proposed for BMI, and because job stressors already have an established relationship with job satisfaction (Bellavia \& Frone, 2005), I would not expect exercise to account for the entire relationship between job stressors and job satisfaction. Therefore, on the basis of the Ego Depletion Model, and in support of the aforementioned research on the 
relationships between physical activity and job satisfaction, I propose that exercise will act as a partial mediator in the job stressor-job satisfaction relationship.

H11d. Exercise will partially mediate the relationship between job demands and job satisfaction.

H11e. Exercise will partially mediate the relationship between skill discretion and job satisfaction.

H11f. Exercise will partially mediate the relationship between decision authority and job satisfaction.

\section{The Mediated-Moderated Effects of Justice on Employee Health}

Research has shown that poor psychosocial work environments, comprised of high demands, low control, and low support, are related to poor health (Belkic et al., 2004, de Lange et al., 2003; Landsbergis et al., 1998). In addition, research has also shown that stressful situations with demands on employees' time and limited resources leave employees feeling a lack of control and with the overwhelming perception that maintaining good health practices is a challenge (Langlie, 1977). Drawing on the Relational Model, we can expect that employees that receive adequate information on upcoming deadlines and have clear and established team goals, would perceive their supervisors to be more procedurally fair. And according to the ERI Model, those that receive the tools and materials needed with which to complete their work tasks, or are approved for their requested time off, may perceive their supervisors as being more distributively fair. These higher justice perceptions may very well engender positive feelings of trust as well as a greater sense of control over their work responsibilities, and both of these things combined 
may increase their internal resources (time, energy, and goodwill), according to the Ego Depletion Model (EDM).

On the basis of the Relational Model, ERI Model and EDM, the interactive effects of higher justice perceptions and lower job stressors would be expected to lead to positive health outcomes in that employees would have the necessary resources to engage in physical activity, resulting in one feeling more positively about their work and supervisor (Conn et al., 2009; Daley \& Parfitt, 1996; Kirkcaldy et al., 1994), as well as it benefitting their weight and BMI levels (Kouvonen et al., 2005; Matarazzo, 1982; Pratt, 1971; Williams \& Wechsler, 1972). Thus, I propose that exercise will at least partially mediate the moderating effect of organizational justice on the relationship between job stressors and BMI and job satisfaction.

$\mathbf{H 1 2 a} / \mathbf{b}$. The interactive effect of procedural (distributive) justice and job demands on BMI will be mediated by exercise.

$\mathbf{H 1 3 a} / \mathbf{b}$. The interactive effect of procedural (distributive) justice and skill discretion on BMI will be mediated by exercise.

H14a/b. The interactive effect of procedural (distributive) justice and decision authority on BMI will be mediated by exercise.

$\mathbf{H 1 5 a} / \mathbf{b}$. The interactive effect of procedural (distributive) justice and job demands on job satisfaction will be mediated by exercise.

$\mathbf{H 1 6 a / b}$. The interactive effect of procedural (distributive) justice and skill discretion on job satisfaction will be mediated by exercise.

$\mathbf{H 1 7} \mathbf{a} / \mathbf{b}$. The interactive effect of procedural (distributive) justice and decision authority on job satisfaction will be mediated by exercise. 


\section{Chapter VII: Method}

In 2007, the Portland State University (PSU) Occupational Health and Safety research team, led by Leslie B. Hammer, Ph.D., Donald Truxillo, Ph.D., and Todd Bodner, Ph.D., began work with the Portland Water Bureau (PWB) to address the balance between work life, safety behaviors, health and supervisor support. The initiative began with several assessments, focus groups, and surveys to understand the work environment and address areas for improvement among its field workers. In the fall of 2011, PSU and the PWB began a supervisor training initiative with the downtown office employees as well. In an effort to expand this important effort and develop more in-depth training and team-based process improvements, PSU also established a partnership with the Portland Bureau of Transportation-Maintenance Operations (PBOT) teams.

With funding provided by the National Institute for Occupational Safety and Health (NIOSH) for three years (2011-2014), the PSU Occupational Health and Safety research team began work with employees in construction and various bluecollar positions from both of the local municipal organizations to improve overall organizational effectiveness, employee morale, job satisfaction, worker health, and safety. The overall initiative was called the 'Safety and Health Improvement Program' (SHIP) and was designed as a multi-step process encompassing an indepth supervisor training, team process meetings, and health assessments. The project plan based on scientific evidence was conducted using a randomized controlled design to roll out the training and health assessments to designated 
groups in the fall of 2012 with follow-up assessments scheduled to take place every six months. The control groups are slated to receive the trainings and assessments at a later time. Employee data were collected during company time via questionnaires completed by the organizational members themselves, followed by a noninvasive health assessment that was conducted by myself and other PSU research assistants. The data for this particular study were collected in the fall of 2012 as part of the baseline data initiative for the overall project that is ongoing through 2014.

\section{Participant Recruitment}

As mentioned previously, participants were recruited from two metropolitan area public agencies in Portland, Oregon. Researchers from PSU met with the management teams and other agency stakeholders from both agencies and devised comprehensive recruitment strategies on how to present the study to the organizational members, roll out the various phases, and minimize contamination of (employees talking about) the process. A flyer (Appendix A) detailing the project, the involvement being requested of each participant, and the benefits to each participant was developed. The flyers were to be posted throughout both agencies as well as utilized as talking points for supervisors to use when discussing the project with their employees. Similar groups were identified across both organizations in terms of the organizational matrices, occupational titles and a cursory understanding of the positions, and then the groups were randomly assigned to either the control or the experimental condition. The final sampling plan 
was chosen to maximize the collection of data from a wide range of employees as well as their supervisors.

Procedure

Weeks prior to the start of the study, the study flyer was provided to both organizations to help communicate the importance of the study and aid supervisors in recruiting as many employees as possible to participate in both the questionnaire and health assessment portions of the study. Recruitment efforts also included PSU researchers going to both organizations to speak to the participants before the start of their shifts during their 'team huddle' meetings. PSU researchers discussed the study, passed out the flyers, and were available for any questions that the participants had.

With regards to data collection, the PSU researchers and agency liaisons coordinated with the various supervisors to schedule a convenient time for the employees to complete the survey and health assessment during their workday while attempting to minimize the overall disruption to their work schedules. The data collection primarily took place in the mornings immediately after the employees completed their morning team huddle meetings.

Data collection for the first organization took place from August - October 2012, while data collection for the second organization took place from October December 2012. As such the data for this study will be treated as archival. Participants were informed that they would be required to complete a paper questionnaire at work, but that participation in the health screening was completely voluntary. As mentioned previously, both components of the data collection were 
scheduled to take place during paid work hours immediately after their morning meetings since all of the employees were already congregated in the same place. First, participants filled out informed consent forms. All participants were given a copy for their records as well. A copy of the informed consent form can be found in Appendix B. Second, the questionnaires were administered individually to all employees and were completed on company time. The questionnaire (Appendix C) was approximately 140 questions long and estimated to take approximately 20-30 minutes to complete. It included questions on employees' perceptions of their work place environment and their supervisor's behaviors, their psychological well-being, their estimated level of health, as well as some demographic questions. The health assessment took approximately 20 minutes and included capturing readings for blood pressure, heart rate, height and weight measures, as well as their body mass index on a health assessment form (Appendix D). All participants were provided a hard copy of their health assessment results at the end of the process, and were given a $\$ 25$ gift card to a grocery store chain for completing both the survey and health screening.

Participant Demographics

The sample group was comprised of 349 participants that ranged from 24 to 71 years of age $(M=44.48 ; S D=9.56)$. Considering the population sampled, it is not surprising that they were primarily male $(89.6 \%$; $=311)$, with on $10.3 \%$ women $(\mathrm{N}=36)$. The participant population was $75.9 \%$ Caucasian, $6.6 \%$ African American, 2.6\% American Indian (or Alaskan Native), 2.6\% Asian, .3\% Native Hawaiian/Pacific 
Islander. Additionally, $8.3 \%$ indicated they were more than one race, and $2 \%$ chose "Other" as their response to the ethnicity question.

With regards to education, 2.3\% completed some high school, 37\% were high school graduates, $47.3 \%$ indicated they had some years of college or trade school, $11.7 \%$ completed a four college, and less than $1 \%$ attended graduate school. In terms of relationship status, $65.3 \%$ of the participants were married, $11.2 \%$ were divorced, $1.1 \%$ was widowed, $10.9 \%$ were living with their significant other, and 9.7\% have never married.

With regards to organizational demographics, $62.5 \%$ were crewmembers, $16.9 \%$ were crew leaders, $8.6 \%$ were supervisors, and $8.9 \%$ chose "Other" as their response for that question. The shortest tenure for this participant group was 2 months (.9\%), and the longest was 33 years (.1\%), with the average being slightly over 11 years $(M=11.06, S D=7.96)$. The overwhelming majority indicated they worked 8 hour days $(\mathrm{N}=192 ; 55 \%)$ and 40 hours per week $(\mathrm{N}=247 ; 70.8 \%)$.

When asked how physically active they are during their workday, $49.6 \%$ participants indicated they are "active" and on their feet most of the day, 37.5\% stated they are "somewhat active" in that they sit half of the day, and $9.5 \%$ indicated they were "not active", and thus, sitting most of the day. When asked how physically active they are during a typical week, the overwhelming majority indicated they were active at least 1 day out of the week, with $21.5 \%$ indicating they are active 5 days per week. Interestingly, however, only $4.9 \%(\mathrm{~N}=17)$ of the sample group had "lean" BMI (BMI <23kg/m²) and 18.3\% (N = 64) had "medium" BMI (BMI in the 23$27 \mathrm{~kg} / \mathrm{m}^{2}$ range). A shocking 20.3\% $(\mathrm{N}=71)$ had "high" BMI $\left(\mathrm{BMI}>27 \mathrm{~kg} / \mathrm{m}^{2}\right)$, 
$32.1 \%(\mathrm{~N}=112)$ had "obesity - class 1" (BMI 30 to $\left.<35 \mathrm{~kg} \mathrm{~m}^{2}\right)$ and $19.5 \%(\mathrm{~N}=68)$ had "obesity - class 2" (BMI > $35 \mathrm{~kg} \mathrm{~m}^{2}$ ).

Measures

Alpha statistics for all measures utilized in this study were above .80, except for the Job Demands and Skill Discretion scales of the 14-item Job Content Questionnaire (JCQ; Karasek, 1985), which had alpha statistics of .72 and .76, respectively. Alpha statistics for all study scales are listed below. A full list of the items used in each of the measures is included in the questionnaire in Appendix C.

Job demands. Job demands refer to work stressors or workload and were measured using the five-item psychological demands subscale (items 10-14) of the JCQ (three of which were reverse coded - items 10,11, and 12). Respondents were asked to indicate the extent to which they agreed or disagreed with statements using a five-point response scale ranging from 1 (strongly disagree) to 5 (strongly agree). An example item includes, "I am not asked to do an excessive amount of work." $(\alpha=.72)$

Job control. Job control refers to the ability to control work activities, and was measured using the nine-item job decision latitude subscale of the JCQ, which can be further broken down into the subscales of skill discretion and decision authority. Responses were asked to indicate the extent to which they agreed or disagreed with statements using a five-point response scale from 1 (strongly disagree) to 5 (strongly agree). Items one through six of the nine-item scale comprise skill discretion $(\alpha=.76)$. A sample skill discretion items is, "My job requires me to be creative." Question 5 in the skill discretion subscale was reverse coded. Decision 
authority is made up of items seven through nine $(\alpha=.88)$. No items in this subscale were reverse coded. A sample decision authority item includes, “On my job, I am given a lot of freedom to decide how I do my work." Incidentally, the two subscales combined have an alpha of .84.

Distributive justice. Distributive Justice was measured using a five-item scale by Moorman (1991), which was originally adapted from Price and Mueller (1986). Respondents were asked to indicate the extent to which they agreed or disagreed with statements using a five-point response scale ranging from 1 (strongly disagree) to 5 (strongly agree). An example includes, "I feel fairly rewarded for the stresses and strains of my job." $(\alpha=.93)$

Procedural justice. Procedural Justice was measured using a seven-item scale by Moorman (1991). Respondents were asked to indicate the extent to which they agreed or disagreed with statements using a seven-point response scale ranging from 1 (strongly disagree) to 7 (strongly agree). An example item is "The PWB/PBOT-MO allows requests for clarification or additional information about the decisions made." $(\alpha=.95)$

Exercise. Exercise was measured using five items modified by Kuehl and colleagues (2012) for the purposes of this study. One stand-alone item asked respondents to indicate the extent to which they were physically active at work ranging from "Not Active" to "Active". Based on the CFA results (see Results section), this single item was used as a control in the BMI regression analyses. There was also a 4-item exercise scale developed by Elliot and colleagues (2004) that asked respondents how many days per week they engaged in hard physical activities, 
moderate physical activities, muscles toning exercises, and physical activities long enough to work up a sweat. The response scale was an eight-point scale ranging from 0 to 7 days. $(\alpha=.82)$

Body Mass Index (BMI). Body mass index (BMI) is a standard height to weight ratio useful for classifying risks associated with weight gain. The World Health Organization (WHO) has developed the following classification for adults: low BMI < $22 \mathrm{~kg} / \mathrm{m}^{2}$; average BMI 23-27kg $/ \mathrm{m}^{2}$; high BMI $>27 \mathrm{~kg} / \mathrm{m}^{2} ;$ obesity $=$ BMI $>30 \mathrm{~kg} / \mathrm{m}^{2}$. BMI was captured using a Tanita electronic body composition scale that utilizes Dual-energy X-ray absorptiometry (DEXA), which sends two X-ray beams with different energy levels through a person's body to measure total body composition and fat content with a high degree of accuracy comparable to the traditional method of hydrostatic weighing.

Job satisfaction. Job satisfaction was measured using a three-item scale based on Hackman and Oldham (1975). Respondents were asked to indicate the extent to which they agreed or disagreed with statements using a five-point response scale ranging from 1 (strongly disagree) to 5 (strongly agree). An example item includes, "You are generally satisfied with the kind of work you do in this job." $(\alpha=.87)$

Demographic variables. Respondents were also asked to provide demographic information on the study survey with regards to their age, gender, highest education achieved, relationship status, tenure with their organization in terms of years and months, and number of hours worked per day/week and their organization role. 


\section{Chapter VIII: Results}

\section{CFA of Job Stressor Variables}

Prior to conducting the main analyses, I conducted a confirmatory factor analysis (CFA) of the job stressor variables based on the JCQ subscales (Karasek, 1998) using Mplus Version 5.21 (Muthen \& Muthen, 2010). As such, I ran 1-, 2- and 3-factor models to determine whether or not the dimensions of occupational stress should be combined into a single construct (job stressors), remain as the two typical conceptualizations of work stress (job demands and job control), or be parceled out into three distinct constructs (job demands, skill discretion and decision authority).

I first tested the one-factor model which views the 14 items as indicators of a single work stress factor. The chi-square test was significant, $\chi^{2}(77, N=349)=$ $875.42, p<.001$, indicating a poor fit to the data. However, the chi-square statistic is problematic as an index of model fit because it is sensitive to sample size (Joreskog \& Sorbom, 1989). Other model fit statistics were thus used to evaluate the fit of the model to the data. As a rule of thumb, Hu and Bentler (1999) suggest that comparative fit index (CFI) values above .95 and root mean square error of approximation (RMSEA) values of .06 or less are indicative of good model fit. Moreover, Kline (1998) suggested that a $\chi^{2} / d f$ ratio of less than two or three is indicative of good model fit. Accordingly, the $\chi^{2} / d f$ ratio was 11.37 , the CFI value was .56 and RMSEA was .17, suggesting that the one-factor model fits the data poorly. 
Next, I tested the two-factor model. Again, the chi-square test was significant, $\chi^{2}(76, N=349)=579.51, p<.001$, indicating a poor fit to the data. The $\chi^{2} / d f$ ratio was 7.63 , the CFI value was .73 and RMSEA was .14 , suggestive of the fact that the two-factor model also fit the data poorly. Lastly, I tested the three-factor model. The chi-square test was once again significant, $\chi^{2}(74, N=349)=379.40, p<$ .001 , the $\chi^{2} / d f$ ratio was 5.13 , the CFI value was .84 and RMSEA was .11 . All the model fit statistics suggest that the three-factor model yielded a marginal fit, however, this model fit better than the other two models. As such, I concluded that it was appropriate to treat the job stressor variables as three separate variables (job demands, decision authority, and skill discretion) for all hypothesis tests.

\section{CFA of Organizational Justice}

Next, I conducted a confirmatory factor analysis of the organizational justice dimensions to determine if procedural and distributive justice should be combined into one justice factor or remain as two separate dimensions. For the one-factor model, the chi-square test was significant, $\chi^{2}(54, N=348)=1,284.72, p<.001$, indicating a poor fit to the data. The $\chi^{2} / d f$ ratio was 23.79 , the CFI value was .68 and RMSEA was .26, suggestive of the fact that the one-factor model also fit the data very poorly. Next, I tested the two-factor model. Although the chi-square test was once again significant, $\chi^{2}(53, N=349)=174.72, p<.001$, all of the other model fit statistics suggested that the two-factor model yielded a better fit $\left(\chi^{2} / d f\right.$ ratio was 3.3, the CFI value was .97 and RMSEA was .06). As such, I concluded that it was appropriate to test procedural and distributive justice as two separate variables. 
CFA of Exercise Variable

As mentioned previously, there were five items in the questionnaire to capture how physically active employees are. The items included an existing 4-item scale modified by Kuehl and colleagues (2012) for the purposes of this study, along with one stand-alone item also created by the researchers. The 4-item scale was originally developed by Elliot and colleagues (2004) for the PHLAME study on firefighters (see reference). The stand-alone item asked respondents to indicate the extent to which they were physically active at work ranging from "Not Active" to "Active", while the 4-item scale asked respondents how many days per week (over the past month) they engaged in hard physical activities, moderate physical activities, muscles toning exercises, and physical activities long enough to work up a sweat. Although the items could have been standardized to fit the same scale, they did not intuitively make sense to me to be combined. The stand-alone item is a broad question that introduces a frame of reference for employees to think about their overall level of physical activity at work. The 4-item scale, on the other hand, asks for very specific information on participants' levels of physical activity throughout the week on specific types of activities (i.e., muscle toning, basketball, swimming, or jogging for at least 30 minutes) with no specification as to whether or not these activities take place within the confines of one's work day or life outside of work. Upon closer inspection of the correlation table, it was evident that the single exercise item shared small significant correlations with the items from the existing 4-item scale, while the relationships among the four items in the scale yielded much higher correlations. Additionally, the reliability analysis of the scale indicated that 
the alpha coefficient was lower with the stand-alone item left in $(\alpha=.76)$ versus taking it out $(\alpha=.82)$. As such, I opted to run a CFA on the exercise variable to determine if, in fact, the single exercise item should remain separate from the existing 4-item scale. I first tested the one-factor model with all five items. The chisquare test was significant, $\chi^{2}(5, N=345)=28.07, p<.001$, and the $\chi^{2} / d f$ ratio was 5.61, the CFI value was .96 and RMSEA was .12, all suggestive of the fact that the one-factor model fit the data very poorly. Next, I attempted to test the two-factor model, however Mplus would not complete the analysis on a one-item factor. Hence, upon inspection of the factor loadings from the one-factor model, I removed the stand-alone exercise item (which incidentally had the lowest factor loading) and reran the CFA on the remaining four items. The chi-square test was once again significant, $\chi^{2}(4, N=346)=11.01, p<.001, \chi^{2} / d f$ ratio was 2.75 , the CFI value was .98 and RMSEA was .11. All the model fit statistics suggest that this model with the four items yielded a relatively better fit than the previous model with all five items. As such, taken on the whole, I concluded that it was appropriate to keep the standalone exercise item separate from the existing exercise scale. Moreover, because of the stand-alone item's correlation with BMI $(r=-.14, p<.05)$, and the fact that weight and physical activity are known controls used in BMI studies, I decided to use the stand-alone exercise item as a control in the BMI regression analyses ("Active at Work").

\section{Correlations among Study Variables}

Means, standard deviations, intercorrelations, and alpha reliability statistics for all study variables are presented in Table 2. An initial examination of the 
correlation table showed that only a few demographic variables were associated with the study variables. For example, age $(r=.11, p<.05)$, relationship status ( $r=-$ $.15, p<.01)$, years of tenure $(r=.21, p<.01)$, hours worked per day $(r=.2, p<.01)$, hours worked per week $(r=.12, p<.05)$, and organizational role $(r=-.24, p<.01)$ were correlated with job demands. Hours worked per day also correlated with skill discretion $(r=.11, p<.05)$ and distributive justice $(r=-.12, p<.05)$. Age $(r=.12, p<$ $.05)$, relationship status $(r=-.15, p<.01)$, years of tenure $(r=.19, p<.01)$, and organizational role $(r=-.23, p<.01)$ were correlated with decision authority. And lastly, gender $(r=-.11, p<.05)$ and ethnicity $(r=-.15, p<.01)$ were correlated with procedural justice.

With regards to the outcomes variables, the data appeared to behave in ways that would be expected given past research findings on the measures used and the proposed relationships in this study. For example, education $(r=-.22, p<.01)$, exercise habits according to the 4-item scale $(r=-.19, p<.01)$, self-reported ratings of activity level at work $(r=-.14, p<.05)$, decision authority $(r=-.18, p<.01)$, and procedural justice $(r=-.12, p<.05)$ were correlated with BMI. This makes sense considering that research has shown that education and SES (of which education is a part) are negatively related to BMI. The more education or higher SES one has, the more one has probably read, studied about, or had access to knowledge and resources to combat negative health behaviors and adverse changes in BMI. Similarly, it is not surprising that exercise is negatively related to BMI, as one would expect that the more one exercises the lower their BMI would be. Furthermore, 
studies have shown that lack of justice and lack of control at work adversely impact one's health (Robbins et al, 2011).

Job demands $(r=-.13, p<.05)$, skill discretion $(r=.39, p<.01)$, decision authority $(r=.32, p<.01)$, procedural justice $(r=.35, p<.01)$, and distributive justice $(r=.45 p<.01)$ were correlated in the expected direction with job satisfaction. These relationships are intuitive in that the more justice and control one has in one's work life, the more satisfaction an employee is likely to experience at work. Conversely, the more demands, less discretion, and less authority one experiences in one's work role, the less job satisfaction they are likely to experience in return.

Not surprisingly, the two justice dimensions were significantly correlated with each other $(r=.43, p<.01)$. Distributive justice also correlated with job demands $(r=-.24, p<.01)$, skill discretion $(r=.30, p<.01)$ and decision authority $(r$ $=.33, p<.01)$. Procedural justice was also correlated with job demands $(r=-.11, p<$ $.05)$, skill discretion $(r=.29, p<.01)$, and decision authority $(r=.31, p<.01)$. One-Way Between Subjects ANOVA

Although performing HLM was not proposed for this study, I anticipated that there might be some significant mean differences in my study variable means based on the sheer fact that the participants came from the two different organizations. As such, I conducted a one-way between subjects ANOVA to compare mean levels of all the study variables across the two different organizations. Overall, there were mean differences on Procedural Justice $\left[\mathrm{F}_{(1,341)}=10.76, p<.01\right)$, partial $\left.\eta^{2}=.03\right]$ and 
Exercise $\left[\mathrm{F}_{(1,345)}=5.03, p<.05\right.$, partial $\left.\left.\eta^{2}=.02\right)\right]$. Although the effect sizes were rather small, the procedural justice perceptions of the PBOT employees appeared to be much lower $(\mathrm{M}=3.15, \mathrm{SD}=1.29)$ than that of the $\mathrm{PWB}$ employees $(\mathrm{M}=3.64, \mathrm{SD}=$ 1.43). Similarly, self-reported levels of physical activity and exercise habits were much lower for PBOT employees $(\mathrm{M}=-.07, \mathrm{SD}=.76)$ than $\mathrm{PWB}$ employees $(\mathrm{M}=.14$, $\mathrm{SD}=.64$ ). As such, all hypothesis tests were conducted using organization (PWB or PBOT) as a control variable.

\section{Control Variables}

In an effort to identify variables that would potentially be useful as additional controls in the regression analyses to follow, I examined the correlation table for all relevant relationships (Table 2). To be considered as a control, a demographic variable had to show a significant correlation with and be theoretically related to the outcome of interest. Surprisingly, job satisfaction was not significantly correlated with any of the demographic variables. Previous research on job satisfaction has controlled for age, gender, and tenure; however, on the basis of no significant correlations among the study variables and for the sake of maintaining maximum power, organization was selected as the only control variable for the job satisfaction hypotheses tests based on the ANOVA results.

BMI was significantly correlated with education $(r=-.22, p<.01)$. Some have suggested that an association exists between education and BMI because environmental conditions associated with low levels of education limit one's access to and knowledge of nutritious food choices and safe means to exercise, it may bring 
on metabolic dysregulation, and trigger higher caloric consumption (Johnson, Kyvik, Skytthe, Deary, \& Sorensen, 2011). BMI was also significantly correlated with exercise - not only participants' ratings on the 4-item exercise scale $(r=-.19, p<$ .01), but also the stand-alone item asking how physically active they believe they are at work $(r=-.14, p<.05)$. Whereas the 4 -item scale is a more appropriate measure of one's physical health behavior pattern, the estimation of how active one thinks they are at work may function more as a proxy for their overall assumption of their health status, and thus be a covariate of BMI. Accordingly, participants' estimation of how physically active they are at work ("Active at Work") was used as a control for all BMI regression analyses, along with education and organization.

\section{Power Analysis}

A power analysis using G*Power 3.1 (Faul, Erdfelder, Lang, \& Buchner, 2009) was conducted to determine a sufficient sample size to achieve statistical power of .80. The power analysis was conceptualized using a hierarchical regression framework with two predictors entered in step 1 (i.e., job strain dimension and organizational justice dimension) and the interaction term entered in step 2 (e.g., job demands X procedural justice). In this context, the effect size $\Delta R^{2}$ represents the unique variance in the outcome variable accounted for by the interaction terms. Due to little to no research regarding the specific justice dimensions as moderators in the relationship between stressors and job satisfaction and BMI, I chose a $\Delta R^{2}$ of .03 as a moderate effect size. $G^{*}$ Power indicated that 368 data points were necessary to detect a $\Delta R^{2}$ of .03 with a power of .80 . I only had 349 data points. Thus, the power analysis was adjusted such that 349 data points yielded an estimated power of 77 . 
Main Analyses

SPSS version 22.0 was used for all main analyses. Hierarchical multiple regressions were conducted to investigate the main effects of job stressors and organizational justice on job satisfaction and BMI. Mediation analyses based on the steps outlined by Baron and Kenny (1986) were also conducted to examine whether or not exercise, at least partially, mediated the relationship of job stressors and job satisfaction or BMI. Next, moderated multiple regression was performed to test if organizational justice moderates the same relationships. Lastly, mediated moderation analyses (Muller, Judd, \& Yzerbyt, 2005) were proposed to see if the interactive effects of justice and job stressors on BMI and job satisfaction were at least partially mediated through exercise.

Results for each proposed hypothesis can be found in Table 1. Results of the specific analyses are presented in Tables 3-20. For the sake of parsimony, I will report the findings related to the job satisfaction hypotheses first, followed by the results related to BMI, and then I will discuss results from the exploratory analyses performed. All significant and non-significant results are also illustrated in Figure 1. Regressions Analyses for Job Satisfaction

Main effects of job stressors on job satisfaction. Hypotheses 1a - 1c, predicted that job demands, skill discretion, and decision authority, respectively, would have significant main effects on job satisfaction. Accordingly, job satisfaction was regressed on organization (control variable) in Step 1, and job demands in Step 2. The same steps were followed for skill discretion and decision authority. The job stressor variables explained significant variance in job satisfaction ranging from $2 \%$ 
to $16 \%$. When controlling for organization, the main effect of job demands was significant $\left(\Delta R^{2}=.01, \beta=-.12, p<.05\right)$, as were the main effects of skill discretion $\left(\Delta R^{2}=.16, \beta=.40, p<.001\right)$ and decision authority $\left(\Delta R^{2}=.11, \beta=.33, p<.001\right)$. Thus, hypotheses H1a - H1c were supported (see Table 3).

The significant negative relationship between job demands and job satisfaction illustrates that employees with reported higher job demands also reported lower levels of satisfaction. Conversely, skill discretion and decision authority both have significant positive relationships with job satisfaction, suggesting that providing employees more discretion and authority within their work roles is related to higher levels of job satisfaction.

Main effects of organizational justice on job satisfaction. Hypotheses $2 \mathrm{a}$ and $2 \mathrm{~b}$ predicted that distributive and procedural justice, respectively, would have significant main effects on job satisfaction. Accordingly, job satisfaction was regressed on organization (control variable) in Step 1, and distributive (procedural) justice in Step 2. When controlling for organization, the main effects of distributive justice $\left(\Delta R^{2}=.20, \beta=.45, p<.001\right)$ and procedural justice $\left(\Delta R^{2}=.13, \beta=.37, p<\right.$ .001) on satisfaction were both significant, signifying that higher perceptions of justice are related to higher levels of satisfaction. Thus, hypotheses $\mathrm{H} 2 \mathrm{a}$ and $\mathrm{H} 2 \mathrm{~b}$ were supported (see Table 4).

In support of previous empirical evidence (Colquitt, 2001), these results also show that higher levels of both distributive and procedural justice are related to higher levels of job satisfaction. 
Organizational justice as a moderator in the job stressor-job satisfaction relationship. Hypotheses $3 \mathrm{a} / \mathrm{b}-5 \mathrm{a} / \mathrm{b}$ predicted that distributive and procedural justice, respectively, would moderate the negative effects that job stressors (job demands, skill discretion, and decision authority, respectively) have on job satisfaction. In order to test these hypotheses, I performed moderated multiple regression analysis. For each regression, the predictor variable (job stressor variable) and the moderator variable (organizational justice dimension) were centered prior to analysis to reduce the potential for multicollinearity. An interaction term was then created by multiplying the centered predictor variable and the centered moderator variable. As such, for each moderated regression analysis, organization (control variable) was entered into Step 1, followed by the mean-centered predictor variables in Step 2 (job stressor variable and justice dimension), followed by the interaction term in Step 3, to test its relationship with job satisfaction.

Hypotheses $3 \mathrm{a}$ and $3 \mathrm{~b}$ predicted that distributive and procedural justice, respectively, would moderate the relationship between job demands and job satisfaction. Results indicate that the interaction terms were not significant, thus, Hypotheses 3a and 3b were not supported (see Tables 5 and 6).

Hypotheses $4 \mathrm{a}$ and $4 \mathrm{~b}$ predicted that distributive and procedural justice, respectively, would moderate the relationship between skill discretion and job satisfaction. The interaction terms were not significant; therefore, Hypotheses 4a and $4 \mathrm{~b}$ were not supported (see Tables 7 and 8 ). 
Lastly, Hypotheses 5a and 5b predicted that distributive and procedural justice, respectively, would moderate the relationship between decision authority and job satisfaction. Results indicate that the interaction terms were not significant, as such, Hypotheses 5a and 5b were not supported (see Tables 9 and 10).

All in all, results from the moderated multiple regression analyses indicate that neither dimension of justice was a significant buffer in the job stressors (job demands, skill discretion, or decision authority) and job satisfaction relationship. Regressions Analyses for BMI

Main effects of job stressors on BMI. Hypotheses $6 a-6 c$, predicted that job demands, skill discretion, and decision authority, respectively, would have significant main effects on body mass index (BMI). Accordingly, BMI was regressed on organization, education, and 'active at work' (control variables) in Step 1, and each of the job stressor variables in Step 2 (three separate analyses were conducted for each IV). When controlling for organization, education, and activity level at work, the main effects of job demands $\left(\Delta R^{2}=.00, \beta=.06, p>.05\right)$ on BMI were not significant. However, the main effects of skill discretion $\left(\Delta R^{2}=.01, \beta=-.11, p<.05\right)$ and decision authority $\left(\Delta R^{2}=.03, \beta=-.18, p<.01\right)$ were. Thus, Hypothesis 6 a was not supported, but Hypotheses $6 \mathrm{~b}$ and $6 \mathrm{c}$ were (see Table 11). These results indicate that while job demands are not related to BMI, higher levels of skills discretion and decision authority are associated with lower BMI.

Main effects of organizational justice on BMI. Hypotheses 7a and 7b predicted that distributive and procedural justice, respectively, would have significant main effects on BMI. Accordingly, BMI was regressed on organization, education, and 
'active at work' (control variables) in Step 1, and distributive (procedural) justice in Step 2. When controlling for organization, education, and activity level at work, the main effects of distributive justice $\left(\Delta R^{2}=.02, \beta=-.13, p<.05\right)$ and procedural justice were significant $\left(\Delta R^{2}=.02, \beta=-.13 p<.05\right)$. Thus, hypotheses $\mathrm{H7}$ a and $\mathrm{H7 \textrm {b }}$ were both supported (see Table 12). These results indicate that higher levels of distributive and procedural justice are associated with lower levels of BMI.

Organizational justice as a moderator in the job stressor-BMI relationship. Hypotheses $8 \mathrm{a} / \mathrm{b}-10 \mathrm{a} / \mathrm{b}$ predicted that distributive and procedural justice, respectively, would moderate the negative effects that job stressors (job demands, skill discretion, and decision authority, respectively) have on BMI. In order to test these hypotheses, I performed moderated multiple regression analysis. For each regression, the predictor variable (job stressor variable) and the moderator variable (organizational justice dimension) were centered prior to analysis to reduce the potential for multicollinearity. An interaction term was then created by multiplying the centered predictor variable and the centered moderator variable. As such, for each moderated regression analysis, organization, education, and 'active at work' (control variables) were entered into Step 1, followed by the centered predictor variables in Step 2 (job stressor variable and justice dimension), followed by the interaction term in Step 3, to test its relationship with BMI.

Hypotheses $8 \mathrm{a}$ and $8 \mathrm{~b}$ predicted that distributive and procedural justice, respectively, would moderate the relationship between job demands and BMI. Results indicate that the interaction terms were not significant, thus, Hypotheses 8a and $8 \mathrm{~b}$ were not supported (see Tables 13 and 14 ). 
Hypotheses $9 \mathrm{a}$ and $9 \mathrm{~b}$ predicted that distributive and procedural justice, respectively, would moderate the relationship between skill discretion and BMI. The interaction terms were not significant; therefore, Hypotheses 9a and 9b were not supported (see Tables 15 and 16).

Lastly, Hypotheses $10 \mathrm{a}$ and $10 \mathrm{~b}$ predicted that distributive and procedural justice, respectively, would moderate the relationship between decision authority and BMI. Results indicate that the interaction terms were not significant. As such, Hypotheses 10a and 10b were not supported (see Tables 17 and 18). All in all, results from these moderated multiple regression analyses indicate that neither dimension of justice moderated the job stressors- (job demands, skill discretion, or decision authority) BMI relationship.

Exercise as a mediator in the job stressors-BMI relationship. Hypotheses $11 \mathrm{a}$ through 11c predicted that the health behavior of exercise would act as a partial mediator in the job stressors-BMI relationship. According to the steps delineated by Baron and Kenny (1986), BMI (objective health outcome) was first regressed on each of the predictors (job demands, skill discretion, and decision authority) in the first equation. If significant, then the second equation states that the mediator (exercise) must then be regressed on the predictor variable (job demands). Had the results of the second equation been significant, I would have moved on to the third and fourth equation whereby BMI would have been regressed on both job demands and exercise to see if the effect of job demands was diminished (or reduced to zero) once exercise was entered into the equation. However, results were not significant when exercise was regressed on each of the predictors, so the third and fourth steps 
were not conducted for any of the regression analyses. Results indicate that exercise did not mediate the relationship between job stressors and BMI. Thus, Hypotheses 11a-11c were not supported (see Table 19).

Exercise as a mediator in the job stressors-job satisfaction relationship. Hypotheses $11 \mathrm{~d}$ through $11 \mathrm{f}$ predicted that exercise would partially mediate the job stressors-job satisfaction relationship. The same steps that were delineated for Hypotheses $11 \mathrm{a}-11 \mathrm{c}$ were followed to test these hypotheses, with job satisfaction as the outcome of interest. Results indicate that exercise did not mediate the relationship between job stressors and job satisfaction. Thus, Hypotheses $11 \mathrm{~d}-11 \mathrm{f}$ were not supported (see Table 20). Together these mediation analyses indicate that exercise was not a relevant linking mechanism in the job stressor-employee health relationship.

Mediated moderated effects of exercise on the job stressor-health relationship. Hypotheses $12 \mathrm{a} / \mathrm{b}, 13 \mathrm{a} / \mathrm{b}$, and $14 \mathrm{a} / \mathrm{b}$ predicted that exercise would act as a mediator between the organizational justice-job stressor interactions and BMI, while hypotheses $15 \mathrm{a} / \mathrm{b}, 16 \mathrm{a} / \mathrm{b}$, and $17 \mathrm{a} / \mathrm{b}$ predicted that exercise would act a mediator between the same relationship and job satisfaction. The hypothesized relationships are termed mediated moderation because the effect of the moderation (organizational justice) on the outcome (BMI or job satisfaction) is believed to be mediated by an additional variable (in this case, exercise). The only difference between classic mediation analysis and a mediated moderation analysis is that the mediated effect is a direct/main effect, whereas in a mediated moderation, the effect is an interaction. Therefore, the first regression analysis must establish the effect of 
interest, in this case, the interactive effect of a job stressor component and organizational justice dimension on the outcome of interest (i.e., BMI or job satisfaction). In order for an effect to be mediated, there must be an effect to begin with. The non-significant organizational justice-job stressor interactions tested previously signified that neither dimension of justice interacted with any of the job stressor variables in their relationships with job satisfaction or BMI. Therefore, Hypotheses $12 \mathrm{a} / \mathrm{b}$ through $17 \mathrm{a} / \mathrm{b}$ are not supported.

\section{Exploratory Analyses}

The following analyses were conducted to more thoroughly explore the relationships among the study variables. First, I conducted additional mediation analyses, followed by additional moderated regression analyses. As none of these relationships were hypothesized a priori and all of the data were captured at the same time, all significant results should be interpreted with caution.

\section{Exercise as a Mediator in the Organizational Justice-BMI Relationship}

Despite the lack of significant findings for exercise as a mediator in the job stressor-job satisfaction/BMI relationship, the health behavior of exercise has received wide support as a mediator in the job stressor-cardiovascular risk factor line of research (Fransson et al., 2012; Nyberg et al., 2013). Results do show significant main effects of both procedural and distributive justice on BMI, and considering that justice (or lack there of) is oftentimes conceptualized as a stressor itself (Judge \& Colquitt, 2004; Kelloway et al., 2005), I decided to explore exercise as a mediator in the relationship between the two justice dimensions and BMI. Unfortunately, results for the second step in the mediation process were not 
significant when exercise was regressed on either procedural justice or distributive justice. In hindsight, I suppose this is not surprising as neither of the justice dimensions shared a significant correlation with exercise (the 4-item scale or the stand-alone item). Therefore, exercise was not found to be a significant a mediator in the relationship between organizational justice and BMI. Organizational Justice as a Mediator in the Job Stressor-Employee Health Relationship Despite the lack of significant results for organizational justice as a viable moderator in the job stressor-employee health relationship, both dimensions of organizational justice shared significantly correlations with all of the study variables. And because organizational justice has a known history of being a relevant stressor itself (Judge \& Colquitt, 2004), as well as a mediator in the job stressor-employee health relationship (Elovainio et al., 2001 \& 2004), I decided to test organizational justice as a mediator in those relationships. Thus, I tested procedural and distributive justice as mediators between job stressors and BMI, and then between job stressors and job satisfaction.

Results indicate that neither distributive nor procedural justice mediates the relationship between job stressors and BMI. Procedural justice does, however, fully mediate the relationship between job demands and job satisfaction, and partially mediates the relationship between skill discretion and decision authority with job satisfaction. A similar pattern was found for distributive justice as a mediator between job stressors and job satisfaction. The specific results for each relationship are described below. 
Procedural justice as a mediator between job demands and job satisfaction.

Results indicate that procedural justice fully mediates the relationship between job demands and job satisfaction $(\beta=.36, t=6.99, p<.001)$. The Sobel test was conducted for the indirect effect, and results indicate the indirect effect is significant (Sobel statistic $=-2.21, p<.05)$. Moreover, the significant direct effect of job demands on job satisfaction $(\beta=-.12, t=-2.25, p<.05)$ was reduced and became non-significant $(\beta=-.07, t=-1.37, p>.05)$ when procedural justice was entered into the equation. The effect of job demands on job satisfaction was thus fully mediated by procedural justice. This result suggests that whether or not an individual interprets their demands at work as procedurally fair is more important for their experience of job satisfaction than the actual amount of demands present at work.

Procedural justice as a partial mediator between skill discretion and job satisfaction. Results also indicate that procedural justice partially mediated the effects of skill discretion on job satisfaction $(\beta=.37, t=7.21, p<.001)$. The Sobel test was conducted for the indirect effect, and results indicate the indirect effect is significant (Sobel statistic $=4.23, p<.001$ ). Moreover, the significant direct effect of skill discretion on job satisfaction $(\beta=.40, t=8.04, p<.001)$ was reduced, yet was still significant $(\beta=.32, t=6.48, p<.001)$ when procedural justice was entered into the equation. The effect of skill discretion on job satisfaction was thus partially mediated by procedural justice. This result suggests that whether or not an individual experiences the level of discretion afforded to them as procedurally fair plays a part in their experience of job satisfaction. 
Procedural justice as a partial mediator between decision authority and job satisfaction. Results also indicate that procedural justice partially mediated the effects of decision authority on job satisfaction $(\beta=.30, t=5.66, p<.001)$. The Sobel test was conducted for the indirect effect, and results indicate the indirect effect is significant (Sobel statistic $=4.40, p<.001$ ). Moreover, the significant direct effect of decision authority on job satisfaction $(\beta=.33, t=6.51, p<.001)$ was reduced, yet was still significant $(\beta=.25, t=4.84, p<.001)$ when procedural justice was entered into the equation. The effect of decision authority on job satisfaction was thus partially mediated by procedural justice. This result suggests that whether or not an individual feels the level of authority they have been afforded is procedurally fair plays a part in their experience of job satisfaction.

Distributive justice as a mediator between job demands and job satisfaction. Results indicate that distributive justice fully mediates the relationship between job demands and job satisfaction $(\beta=.44, t=8.88, p<.001)$. The Sobel test was conducted for the indirect effect, and results indicate the indirect effect is significant (Sobel statistic $=-4.08, p<.001)$. Moreover, the significant direct effect of job demands on job satisfaction $(\beta=-.12, t=-2.25, p<.05)$ was reduced and became non-significant $(\beta=-.02, t=--.39, p>.05)$ when distributive justice was entered into the equation. The effect of job demands on job satisfaction was thus fully mediated by distributive justice. This result suggests that whether or not an individual interprets their demands at work as distributively fair is more important for their experience of job satisfaction than the actual amount of demands present at work. 
Distributive justice as a partial mediator between skill discretion and job

satisfaction. Results also indicate that distributive justice partially mediated the effects of skill discretion on job satisfaction $(\beta=.36, t=7.41, p<.001)$. The Sobel test was conducted for the indirect effect, and results indicate the indirect effect is significant (Sobel statistic $=5.06, p<.001)$. Moreover, the significant direct effect of skill discretion on job satisfaction $(\beta=.40, t=8.04, p<.001)$ was reduced, yet was still significant $(\beta=.29, t=5.96, p<.001)$ when distributive justice was entered into the equation. The effect of skill discretion on job satisfaction was thus partially mediated by distributive justice. This result suggests that whether or not an individual experiences the level of discretion afforded to them as distributively fair plays a part in their experience of job satisfaction.

Distributive justice as a partial mediator between decision authority and job satisfaction. Results also indicate that distributive justice partially mediated the effects of decision authority on job satisfaction $(\beta=.30, t=5.66, p<.001)$. The Sobel test was conducted for the indirect effect, and results indicate the indirect effect is significant (Sobel statistic $=5.51, p<.001)$. Moreover, the significant direct effect of decision authority on job satisfaction $(\beta=.33, t=6.51, p<.001)$ was reduced, yet was still significant $(\beta=.21, t=4.03, p<.001)$ when distributive justice was entered into the equation. The effect of decision authority on job satisfaction was thus partially mediated by distributive justice. This result suggests that whether or not an individual feels the level of authority they have been afforded is distributively fair plays a part in their experience of job satisfaction. 
Exercise as a Moderator

On the basis of previous research on the wide-ranging benefits of exercise to offset mental health issues (Rethorst et al., 2009) and physical health ailments (Nyberg et al., 2013), I tested exercise as a potential moderator to further understand the existing relationships between exercise, job stressors, organizational justice and the outcomes of interest (job satisfaction and BMI). I conducted moderated regression analyses to explore whether or not exercise interacted with job stressors or organizational justice to impact employee health. Specifically, I tested to see if exercise would moderate the effects that job demands, skill discretion, decision authority, distributive and procedural justice have on job satisfaction, followed by an exploration of those same relationships on BMI. Interaction of Exercise and Job Demands on BMI

Results indicate a significant interaction between job demands and exercise on BMI ( $\beta=.13, t=2.48, p<.05)$, illustrating that exercise interacted with job demands to explain 2\% of the variance in BMI. As one might expect, BMI levels change as a function of one's job demands and how physically active one is. Contrary to one's expectation, exercise has an interesting and unexpected relationship with high job demands. Results show that for employees with reported high demands, BMI is actually the highest for the high exercisers.

Simple slope tests were conducted to interpret the interaction effect of exercise and job demands on BMI at varying levels of exercise (low, moderate, high). The purpose of this last step is to test each line (for example, below the exercise mean and above the exercise mean) to determine if there is a significant 
relationship to the criterion for each subset of the interaction effect. With a mean level of exercise for this participant group reported to be 2.65 days, low exercisers were individuals that reported exercising zero days out of a possible seven, moderate exercisers exercised 3.5 days out of seven, and high exercisers exercised seven out of seven days. Results from the simple slope tests indicate that for low exercisers there were no significant differences in BMI regardless of their reported levels of job demands $(t=1.29, p>.05)$. However, there were significant differences in BMI for both moderate exercisers $(t=2.67, p<.01)$ and high exercisers $(t=2.96$, $p<.01$ ). The graphical representation of these relationships can be found in Figure 2 and a more comprehensive explanation of these relationships is provided in the following chapter (Chapter 9: Discussion).

Interaction of Exercise and Distributive Justice on Job Satisfaction

Results of the moderated regression analyses also indicate a significant interaction between distributive justice and exercise $(\beta=.15, t=3.08, p<.01)$ on job satisfaction, illustrating that exercise interacted with distributive to explain $2 \%$ of the variance in job satisfaction. These findings demonstrate that job satisfaction changes as a function of how physically active these employees are and how distributively fair they believe their supervisors to be. Simple slope tests were again conducted to interpret the interaction effect of exercise and distributive justice on job satisfaction at varying levels of exercise (low, moderate, high). Similar levels of the moderator were used to distinguish between low exercisers ( 0 days), moderate exercisers (3.5 days), and high exercisers (7 days). Results from the simple slope tests indicate that there are significant differences in job satisfaction at the different 
interaction effects: low exercisers $(t=11.07, p<.001)$, moderate exercisers $(t=$ $4.18, p<.001)$, and high exercisers $(t=3.32, p<.001)$. More specifically, the highest levels of job satisfaction are experienced by the high exercisers with high reported levels of distributive justice. A graphical representation of these relationships is found in Figure 3 and a more comprehensive explanation of these relationships is presented in the following chapter (Chapter 9: Discussion).

\section{Summary of Findings}

With regards to the main effects on job satisfaction, it was found that all of the predictor variables are significantly related to job satisfaction. More specifically, the significant negative relationship between job demands and job satisfaction illustrates that employees with reported higher job demands also reported lower levels of satisfaction. Conversely, skill discretion and decision authority both have significant positive relationships with job satisfaction, suggesting that providing employees more discretion and authority within their work roles is related to higher levels of job satisfaction. In addition, results also illustrate that higher perceptions of both distributive and procedural justice are related to higher levels of job satisfaction.

With regards to the main effects on BMI, results indicate that job demands are not related to BMI, however, job control (skills discretion and decision authority) and organizational justice are. These results indicate that higher levels of skill discretion, decision authority, distributive justice and procedural justice are associated with lower levels of BMI. 
Despite being touted as a relevant organizational factor that may buffer the job stressor-employee health relationship (Cropanzano et al., 2005), results from the moderated multiple regression analyses indicate that neither dimension of justice moderated the relationships between job stressors (job demands, skill discretion, or decision authority) and the outcomes of interest in this study (job satisfaction and BMI).

Results from mediation analyses indicate that exercise did not mediate the relationships between job stressors and job satisfaction/BMI. Together these mediation analyses indicate that exercise was not a relevant linking mechanism in the job stressor-employee health relationship.

Exploratory analyses were conducted to further investigate the nature of the relationships between the study variables. Considering the significant main effects of both procedural and distributive justice on BMI, and the fact that justice (or lack there of) is oftentimes conceptualized as a stressor itself (Judge \& Colquitt, 2004; Kelloway et al., 2005), I tested exercise as a mediator in the relationship between organizational justice and BMI. Results, however, indicate that exercise was not found to be a significant a mediator in the relationship between organizational justice and BMI.

Next I tested organizational justice as a mediator in the job stressoremployee health relationship. Results indicate that neither distributive nor procedural justice mediates the relationship between job stressors and BMI. However, both distributive and procedural justice fully mediate the relationship between job demands and job satisfaction, suggesting that whether or not an 
individual interprets their demands at work as procedurally and distributively fair is more important for their experience of job satisfaction than the actual amount of demands present at work. Results also found that distributive and procedural justice partially mediate the relationship between skill discretion and decision authority with job satisfaction. These results suggest that whether or not an individual feels the level of discretion and authority they have been afforded is procedurally and distributively fair plays a part in their experience of job satisfaction.

The last set of exploratory analyses included moderated regression analyses to explore whether or not exercise interacted with job stressors or organizational justice in the prediction of employee health. Results indicate a significant interaction between job demands and exercise on BMI, illustrating that BMI levels change as a function of one's job demands and how physically active one is. Simple slopes tests showed that the relationship between job demands and BMI is not significantly impacted by low exercisers. Contrary to one's expectation, however, BMI is actually the highest for the high exercisers with reported high levels of job demands. A significant interaction was also found between distributive justice and exercise on job satisfaction. As expected, the highest levels of job satisfaction are experienced by the high exercisers with the highest reported levels of distributive justice. A summary of all hypothesized and exploratory analysis results can be found in Table 1. 


\section{Chapter IX: Discussion}

In investigating the complex relationships between job stressors, organizational justice, health behaviors and employee health, this study is able to provide preliminary empirical data showing that job stressors, justice, and exercise are in fact associated with employees' levels of job satisfaction and BMI. This study aimed to investigate whether or not job stressors are related to employees' subjective well-being (job satisfaction), as well as their physical health (BMI), and if organizational justice moderated those relationships. Further, I aimed to investigate if the interaction of job stressors and organizational justice on job satisfaction and BMI was mediated by the health behavior of exercise. Understanding the bigger picture of how work stressors and perceptions of organizational justice are associated with employee health can help researchers and practitioners understand the far-reaching impact that job design and leadership practices can have on the well-being of their workforce (Colquitt et al., 2001; Robbins et al., 2012). The Relationship Between Job Stressors and Job Satisfaction and BMI

Results show that all of the hypothesized relationships between job stressors and job satisfaction were significant and in the expected direction. Accordingly, employees tend to report higher levels of job satisfaction when their job demands are low, and when they have higher levels of skill discretion and decision authority. These results largely corroborate previous findings on the some critically important antecedents to employee job satisfaction (a proxy for subjective well-being) (Colquitt et al., 2001; Kivimaki et al., 2008). 
With regards to the objective health outcome of BMI, results show that job discretion and decision authority are related to employees' BMI levels, supporting previous research findings on the relevance of high job control to one's health, and lack of control to ill health (Bosma et al., 1998). Although it was surprising that job demands was not significantly related to BMI, the correlation table also provided no evidence of an association between the two.

The Relationship Between Organizational Justice and Job Satisfaction and BMI

In light of the surge of research interest in the role of organizational justice within the workplace, this study examined the relationship between two dimensions of organizational justice (procedural and distributive) and employee subjective well-being, as well as their objective physical health. Results show that higher levels of job satisfaction are associated with higher levels of procedural and distributive justice, further corroborating previous findings on important antecedents to employee job satisfaction (Colquitt et al., 2001; Kivimaki et al., 2008).

Perhaps the most important contribution of this study is the investigation of the distinct and unique relationships between procedural and distributive justice and BMI. Although justice or lack of fairness is becoming more common to investigate in the workplace, its relationship with objective health outcomes is still scarce in the literature, as noted by Robbins and colleagues in their recent metaanalysis (Robbins et al., 2012). Accordingly, results of this study provide cutting edge, empirically based evidence on the relevant, but scarcely investigated relationship between procedural and distributive justice and their relationships with BMI (Elovainio et al., 2001; Gimeno et al., 2010; Nyberg et al., 2012; 2013; 
Robbins et al., 2012).

In addition to the direct relationship between justice and subjective/ objective health outcomes, I also examined the interactive relationships between job stressors and organizational justice and their impact on employees' reported levels of job satisfaction and BMI - all relationships that have not been studied in this particular combination before - to further add to our knowledge of the list of organizational safeguards in the job stressor-health relationship. Despite being touted as a relevant organizational factor that may buffer the job stressor-employee health relationship (Cropanzano et al., 2005), results from the moderated multiple regression analyses indicate that neither dimension of justice moderated the relationships between job stressors (job demands, skill discretion, or decision authority) and the outcomes of interest in this study (job satisfaction and BMI). Although the data did not support my predictions, I did, however, discover in post hoc analyses that organizational justice was a significant mediator in the relationships between job stressors and job satisfaction. Distributive and procedural justice fully mediated the relationship between job demands and job satisfaction, and partially mediated the relationships between skill discretion and decision authority and job satisfaction. These results fall in line with previous findings on the mediating role of justice in the relationship between work characteristics and selfreported emotional reactions and job satisfaction (Elovainio et al., 2001; Elovainio et al., 2004; Robertson, Moye, \& Locke, 1999). Results also support classic theories on stress that emphasize individual evaluative processes as intervening processes between the environment and individual reactions (Lazarus \& Folkman, 1984). For 
example, Katz and Kahn's (1966) Michigan model of occupational stress suggested that employees' subjective perceptions mediate the influence of work characteristics on their levels of job dissatisfaction, psychic distress, chronic disease, and poor self-rated health.

As mentioned previously, job satisfaction and BMI are two important outcomes in the work stress literature and within the workplace context that need to gain more traction if they are to be taken seriously by employers and organizational management with their eye on healthcare premiums and bottom line figures (Robbins et al., 2012; Simons \& Roberson, 2003). With empirical evidence for a relationship between organizational justice and job satisfaction as well as BMI, interventions targeted at addressing supervisors' knowledge and awareness of the benefits of fairness in the workplace may be an initial preventative step to adverse BMI changes, the lowering of employees' job satisfaction levels, and the onset of serious long-term health outcomes. Implementing such interventions would fall seamlessly in line with the overall goals of the NIOSH Total Worker Health program that advocates for health protection and health promotion by targeting risk factors for preventable chronic health conditions in the workplace, identifying more efficient uses of resources, and gaining a deeper understanding of the underlying connections between the health, safety and performance of workers to the overall health of the organization (Hammer \& Sauter, 2013; Schill \& Chosewood, 2013). The Role of Exercise

Although the key mediators in the association between job stressors and cardiovascular disease risk factors have long been debated and explored, I 
hypothesized that exercise would at least partially mediate the relationships between job stressors and job satisfaction/BMI based on meta-analytic data on 47,000 men and women showing that job stressors affect CHD via poor health behaviors and the increasing risk of diabetes (Nyberg et al., 2013). While none of the meditational hypotheses of exercise were supported, I investigated exercise as a possible moderator in those relationships considering its significant correlation with BMI $(r=-.19, p<.001)$ and its association with job stressors in the literature (Fransson et al., 2012).

Results from the moderated regression analyses and simple slope tests show that BMI levels change as a function of the interaction between moderate and high levels of exercise and high levels of job demands. For employees with reported low job demands, however, BMI levels did not change significantly as a function of how much one exercises (low versus high exercisers). This finding may loosely be interpreted as low levels of job demands are ideal for maintaining a (relatively) lower BMI. However, exercise has an interesting and unexpected relationship with high job demands, at least for this sample. Results show that for employees with reported high demands, BMI is actually higher for individuals that reported being more physically active (moderate and high exercisers). One theoretical explanation for this relationship is the notion of Festinger's theory of cognitive dissonance (1957) - whereby an individual may deal with his internal conflict of having high BMI with a tendency to over estimate how busy he is at work as a rationale for the elevated BMI (“Work makes me fat!"). Similarly, individuals experiencing this internal psychological distress may have a tendency to over inflate how much they 
actually exercise as a coping mechanism to make themselves feel as though they are doing everything they can to help their situation ("I work out all the time. Work must be making me fat.").

Another explanation that comes to mind is that individuals that have higher levels of job demands are in fact more physically active, and they engage in that physical activity to offset those feelings of stress (i.e., "sweat out" their problems). But all of the benefits of the physical activities they engage in may be offset by the fact that these busy and stressed out individuals aren't making the correct choices when it comes to their diet or other lifestyle behaviors. And perhaps the calories consumed throughout one's busy day far outweigh the calories being expended, resulting in the unexplained increase in BMI.

Yet another explanation for this unexpected relationship may be that employees in these positions mistakenly believe that they are highly active due to the very nature of their presumably physical jobs and the fact that they work in the field compared to someone who works in an office all day. In reality, however, it's possible that these individuals exert more psychological and emotional energy than actual physical energy going from job to job. Additionally, the fact that one's entire day is spent in transit and/or out in the field may suggest that these individuals likely have to grab food on-the-go or may have to resort to drinking sodas and sugary, caffeinated drinks for quick bursts of energy throughout the day. Incidentally, Payne and colleagues found that high stress jobs have more of a direct impact on healthy eating than decisions on whether or not one should exercise. More specifically, the researchers found that jobs with high demands and low 
control may only affect consumption of 'high-density' foods rather than 'healthy' foods (Payne et al., 2005).

Although there is a big difference between being active at work versus how often one exercises during one's day, the terminologies were likely perceived by the study participants to be interchangeable given the nature of the context within which they work (out in the field several days per week). Even more different is how active someone is off work and during his or her leisure time, although that was not captured in this study. While there is no way to truly know how physically active a participant is with a self-report survey, the descriptive statistics illustrate that there is some discrepancy in how the participants view themselves and their physical activity levels. For example, $50 \%$ of the participants view themselves to be active while at work (defined as 'on my feet most of the day'). And when asked how many days in a typical week they engage in physical activity long enough to work up a sweat, 75 (the highest number of candidates per response option) indicated at least five days per week, more than likely signifying that they perceive themselves to be active all (work) weeklong. However, when asked how many days per week they engage in hard physical exercise that makes them sweat and breathe hard for at least 30 minutes, results indicate that 70 (the highest number of candidates per response option) chose zero days per week.

Moderated regression analyses also indicate a significant interaction between distributive justice and exercise on job satisfaction, illustrating that job satisfaction changes as a function of how physically active these employees are and how distributively fair they believe their supervisors to be. On the basis of the Effort 
Reward Imbalance Theory (1996), employees with higher perceptions of distributive justice presumably feel rightly compensated for their work efforts, which is associated with higher levels of satisfaction. These elevated and positive emotions toward their supervisor or work in general may leave these employees feeling stocked and/or replenished in their resources, which allows them to engage in physical activities and hobbies of their choice outside of work. Insights from Fredrickson's Broaden-and-Build Theory (1998) posit that positive emotions cultivate cognition and behavioral repertories and that upward spirals of positive emotions help to counter downward spirals of negativity (Garland, Fredrickson, Kring, Johnson, Meyer, \& Penn, 2010), with the idea that the more one works out the better they feel, and the better feel the more they work out. A similar scenario applies to the low exercisers who reported lower levels of job satisfaction. These individuals may be caught up in a negative spiral whereby they consistently ruminate on undesirable and destructive thoughts of how unfair their boss is and how miserable they are at work. These types of thoughts are a drain on one's resources inhibiting them from wanting to engage in physical activity. Animal and human studies have found that exercise-trained individuals exhibit an attenuated HPA axis response to physical and mental stress, and that this physiological mechanism has the potential to influence the relationship between physical activity and mental health states (Luger, Deuster, Kyle, et al., 1987). Moreover, Ossip-Klein and colleagues (1989) found that both aerobic and resistance training resulted in a decrease in depressive symptoms as well as enhanced self-esteem, which was attributed to improved body image and an increased sense of mastery. 
These results corroborate earlier findings, which state that exercise plays an important role in the treatment of depression and anxiety, as well as in the prevention of obesity and cardiovascular disease. The American College of Sports Medicine has recommended that overweight and obese individuals engage in aerobic exercise for at least 150 minutes/week, eventually increasing it to 200-300 minutes/week (Jakicic et al., 2001). Although this recommendation is intended for the general population, the researchers acknowledge the unlikelihood that it will ever be adopted by policy-makers and practitioners targeting issues of obesity in the workplace because of inherent constraints in worksite settings (time, budget, resources). Taking into consideration the multitude of significant results related to anthropometric outcomes in the meta-analysis conducted by Conn and colleagues (2009), the worksite presents extensive opportunities to reach large numbers of people for health promotion and disease prevention, given that most of the adult population is employed (Atlantis, Chow, Kirby, Fiatarone Singh, 2006).

\section{Practical Implications}

Although the results from the moderated regression analyses and mediation analyses are interesting, plausible, and timely for implications on the workplace, results should all be treated with caution as none of the relationships were proposed a priori and because of the cross-sectional nature of the data collection. However, the significant relationships between job stressors and organizational justice with both of my outcomes of interest - job satisfaction and BMI - should illustrate to organizational leaders the full effects that stressful work environments can have on their employees' health and well-being, as well as the ramifications of 
preserving such a toxic climate. Empirical evidence on these relationships should provide organizational leaders a better perspective on how their leadership practices and the work climate they create directly impacts their employees' time, resources, health and happiness. Add to that the effects of prolonged stress experienced from the unfair treatment from one's supervisor, and the detrimental effects may start to spill over into other domains of individuals' lives (Hammer et al., 2005a; Meijman, Mulder, \& Van Dormolen, 1992; Totterdell et al., 1995).

Elovainio and colleagues (2004) suggested that a perceived lack of organizational justice indicates a general lack of trust, problems with managerial procedures, and glitches in everyday dynamics between employees and their supervisors. Considering the influence that supervisors have over their employees, it is not surprising that they have a direct hand in the things that impact their employees' psychological and physical health. Based on my research findings, there are a number of things that employers implementing organizational interventions aimed at increasing justice perspectives can do - be it the timely sharing of information that supervisors are privy to from upper management (i.e., impending company merger; upcoming openings of several internal positions), the equitable allotment of resources to which they have access (i.e., cell phones for all employees; funds to send employees to conferences), and the instrumental supportive roles supervisors can play in their employee's work lives by providing them guidance or even access to an organizational mentor.

Additionally, supervisors assign workloads and task assignments, and, thus, are responsible for job design and the demands bestowed upon their employees. 
Supervisors should design ways to implement a democratic system of assigning work so as to not overburden a certain individual(s), as well as a means for shifting work from one worker to another if an emergency situation arises. Similarly, as they are the ones to grant their employees the discretion and authority to conduct their jobs in a manner that suits them best, supervisors can capitalize on their employees' self-guided approaches by looking to them as the experts on 'how' to conduct the work as well as how best to create training manuals, establish team protocols, organizational policies, and industry standards. And though supervisors may not always be the ones responsible for implementing (unfavorable) company policies, they are certainly in a position to provide their employees a medium for discussing their opposition to a policy and suggestions for solutions (i.e., how can employees without children also benefit from work-family policies?). All of these factors highlight the notion that supervisors choose the types of relationships to have with their employees and the work environment they instill and cultivate within their workgroups. Most notably, they play a sizable role in influencing employees' perspectives on how stressful and evenhanded their work environment is, and in turn, how justice perspectives may be the direct and indirect conduits between work characteristics and employees' psychological and physical health (Kelloway et al., 2005; Robbins et al., 2012).

Importantly, these findings provide an avenue for supervisors and employees to work together to enhance their existing working relationships in order to compensate for workplace deficiencies (i.e., intra-departmental communication; teamwork processes; outdated/limited employee policies; job with 
little autonomy; inability to expand one's role; flat organization; lack of funds) in an effort to create a more satisfying workplace environment (i.e., increasing job control, job-sharing, flexible schedules). Similarly, organizations can take on a more global, proactive approach to change their organizational culture to encompass more of a justice climate (Naumann \& Bennett, 2000). With this type of paradigm shift, employers can alter their focus away from trying to 'fix' their workers to permanently changing the work environment by introducing worthwhile companywide programs, such as employee work stress and health interventions, teamwork activities, teambuilding meetings and outings, various supervisor training opportunities (i.e., family supportive supervisor behaviors, interpersonal, communication, conflict resolution, performance appraisal and feedback training, mentor programs), as well as an overall transformation in the way in which performance reviews, salary increases and bonuses are administered and allocated. While most workplace interventions place a large portion of the burden on individuals to fix themselves or to reshift their appraisal of how stressful their work environment really is (Lazarus \& Folkman, 1984), those actions do not get at the root cause of the problems found within work groups or directly between an employee and his or her supervisor. Conversely, Heaney (2003) argues that always assuming that an organizational level change must take place (i.e., job design alternatives, climate shift) takes any and all accountability away from individuals that knowingly abuse their body (smoking, poor diet, lack of exercise) and their privileges (flexible work schedules, remote positions, etc.). Accordingly, organizations should do what they can to assess the situation, conduct a needs 
analysis with a representative sample from the workforce, and then implement a feasible, transparent, and effective work solutions, as Heaney (2003) has pointed out that loose interventions just simply don't work and don't last. Moreover, research has shown that interventions targeted at managers and supervisors tend to work better than other types and may even have an impact on individual and organizational costs associated with their employees (e.g., absenteeism, productivity, turnover) (Hurrell, 2003). In following this approach, organizations may want to consider ways of enhancing supervisor-employee relationships and communication channels. For example, efforts can be made to increase levels of leader-member exchange in dyadic relationships, supervisors can attend training to enhance their communication skills, or company-wide policies that are clear, upfront, and transparent can be put into place that promote and enhance employees' justice perceptions.

Further, research by McLeroy, Gotttlieb, and Heaney (2002) posit that supervisor interventions aimed at reducing interpersonal tension and conflict, strengthening social ties, and clarifying goals and expectations may be expected to decrease unnecessary stress and increase employee health. While such an approach is considered person-oriented with regard to supervisors, it is viewed as environment-oriented from the employees' perspectives. According to Hurrell (2003), all of these changes will provide more consistent effects than psychosocial interventions because they address more of the structural issues rather than trying to 'fix or train' employees to be more flexible, adaptable, tolerant to stress, etc. 
Hand in hand with this proactive approach (job modification and manager training), however, should be a companywide campaign for individual health promotion because organizations can't just focus on reducing work stressors and modifying jobs and assume that is the panacea to a healthier work environment. As researchers and practitioners ourselves, it behooves us to try to get employees onboard with seeing the value in leading healthier lifestyles for themselves. This is particularly important to consider in light of the fact that approximately $50 \%$ of the sample has high BMI or higher. These data suggest that a sedentary workday may have become the norm for this overweight sample and that supervisors may actually need to focus their efforts on health promotion interventions. For example, supervisors may choose to incorporate mandatory lunch breaks, and/or scheduled times for employees to engage in physical activity (i.e., stretching immediately after their morning huddles). They could conduct meetings while going on a walk with their teams and they could encourage their employees to walk to nearby work locations rather than driving. They could even go as far as to try to understand the reasons behind their employees' stress eating habits. It is important to note, however, that organizations can spend thousands of dollars to install a gym on their premises, and they can even offer free gym memberships to their employees, but at the end of the day, they cannot force their employees to actually engage in physical activity. Similarly, organizations can provide healthy snacks for its workforce, but they cannot actually make the employees eat them. Organizational leaders can only do so much, and then the onus shifts over to the individual employee to engage in physical activity and choose the healthy food options. 
It probably goes without saying that before any program is implemented or policy put into effect, organizational leaders and stakeholders should get input from a representative sample of their employees on what types of changes would help to reduce their work stressors and subsequent adverse health outcomes. Not only would organizations be allowing their workforce a "voice" (Thibault and Walker, 1975), but getting employees' input would also likely increase the amount of buy-in from the actual workforce. This was evident in the meta-analytic results by Conn and colleagues (2009) that showed that in terms of the anthropometric outcomes (one of which was BMI), there were significant effect sizes for interventions delivered at the worksite, interventions on company paid time, interventions designed by company employees themselves, and the presence of a fitness facility onsite in the workplace. Conversely, the researchers did want to stress that despite the mixed results on improved work attendance, job satisfaction, and job stress, even modest reductions in absenteeism, for instance, may result in substantial fiscal savings when multiplied by many employees.

\section{Potential Limitations}

Despite my best efforts to design and conduct a theoretically sound and methodologically robust study, all study designs undoubtedly suffer from some shortcomings. As Kasl (1987) noted, a dilemma oftentimes faced by many researchers is that "better research designs are more a function of the resources available to the investigator and less a reflection of his/her level of methodological sophistication." While large data sets, a large, representative sample, a good response rate, and the ability to identify and control for a variety of confounding 
factors are all considered important advantages and strengths, researchers conducting field research must always acquiesce to certain limitations within the confines of reality, all of which bring their fair share of distinct disadvantages.

That said, this study did have some limitations that should be noted. First, one could argue that as a result of the sample group of blue-collar employees from the construction industry there may be a lack of generalizable results. Participation in the questionnaire portion was not voluntary, but participants were able to opt out of the health assessment, and thus differential selection factors (i.e., impression management, motivation to earn the incentive, work avoidance) could have impacted the observed findings. Additionally, as Theorell and his colleagues (2003) point out, "while it is unlikely that individuals with CHD would select themselves into high strain occupations, it is plausible that individuals previously employed in high strain jobs that experienced cardiovascular symptoms moved into passive isolated jobs."

Second, due to the restrictions of the study parameters, all of the variables of interest in this study were evaluated on the basis of only a single point in time. However, the mixed-source, mixed-method design employed in this study is helpful in offsetting the potential limitations of common method bias that typically occurs with exclusively survey-based research (Podsakoff et al., 2003). As many researchers have noted, stress, strain, justice (or lack thereof), satisfaction and BMI are likely the result of culminating effects. Thus, it is virtually impossible to paint an accurate and full picture of someone's reality by just capturing one snapshot in time. The ideal design would encompass a longitudinal, panel design, which would allow 
for the measurement of three kinds of causal relationships: standard cross-lagged effects (i.e., the effects of demands at Time 1 on strain related outcomes (health) at Time 2), as well as reversed and reciprocal relationships; all of which would provide researchers with the most accurate portrayal of individuals' true experience of stress and strain and help researchers better understand the causality of occupational stress and justice effects more rigorously. This would be especially relevant when addressing health outcomes, as some effects on health and well-being only become apparent over long stretches of time.

Third, many work stress studies to date have been restricted to males (Robbins et al., 2012). And while the aim of this study is to collect data on both males and females, the sample was predominantly male, thereby perpetuating the problem and further flooding the research arena with male-centric results. Finally, despite the use of objective health assessments to obtain some of the data, this study also relied on survey questions to evaluate job stressors, organizational justice, and subjective well-being to measure individuals' perceptions regarding their work environment, work relationships, and overall health. As such, it is possible that differences in readability, motivation, fatigue, personality, mood, stress level, and sensitivity to justice led to differences in the perception of these constructs.

Lastly, it is important to note that the scales used to measure exercise (or physical activity) were likely not appropriate for this sample considering the nature of their jobs within the construction industry and the fact that half of the participants (50\%) viewed themselves to be active at work. There is a level of subjectivity and impression management that is likely involved when you ask an 
individual to self-report how active they are at work when they work a 'field' job versus an 'office' job. In addition, the stand alone single item that asked participants how active they perceived themselves to be at work was so different from the four questions that asked how many days (from 0 to 7 ) they engaged in vigorous, moderate, and muscle toning exercises, that it did not make sense to standardize and combine them into one scale. Moreover, as mentioned previously, being active at work means different things to different people, as we all have different conceptualizations of what constitutes 'being active'. Not only that, but being active at work is vastly different than exercising during one's word day, which is even different yet from how active one is outside of the work context.

All of these factors highlight the fact that the ability to demonstrate a relationship between physical activity and health benefits is largely dependent on measures of physical activity and specific health outcomes that are accurate, precise, and reproducible (NCHS, 1989). Even though measures of physical activity have improved over time, most research to date still relies on self-reports, such as diaries, logs, and recall surveys. While they are relatively easy to administer, inexpensive and acceptable to most study participants, these mediums are subject to recall bias and nonrepresentative sampling of activity depending on when individuals are asked (DHHS, 1996). Direct monitoring, on the other hand, eliminates many of the limitations associated with self-reported measures. However, it is expensive, timeconsuming, and may be burdensome to all involved (participants, surveyors, researchers). It goes without saying that the medium chosen to measure physical activity should match the intended purpose and overall goal of the study. 
Suggestions for Future Research

The goal of this dissertation was to explore the role of organizational justice and health behaviors in the relationship between job stressors and employee health. To that end, the interesting relationships uncovered among the study variables should be further explored. For example, with exercise as a significant moderator in the job stressor-employee health relationship, it would worthwhile to further drill down on the concept of exercise among this sample type. It is possible that with this group being from the construction industry and conducting primarily manual tasks, that the participants have a completely different conceptualization of what 'being active at work' means. It is also possible that there will continually be a floor effect with this type of sample if they inherently feel as though there is no need to exercise outside of work as a result of how physically active they perceive themselves to be at work. As such, it would be prudent for researchers working with these types of samples to understand the job they do, to better operationalize different activity levels and calibrate participants' understandings of the various levels, to employ a measure that more accurately captures what they do on the job, and to distinguish between activity levels during the work day and outside of work.

In light of recent meta-analytic results (Nyberg et al., 2013) suggesting that the linking mechanisms between high stress jobs and cardiovascular disease risk factors is physical activity and glucose levels (onset of diabetes), it would be worthwhile to also explore the effects of diet in conjunction with physical activity as research shows that high stress jobs have more of an impact on healthy eating than on whether or not someone chooses to exercise (Payne et al., 2005). This may entail 
asking participants to keep food logs, surveying them on their eating habits, trying to understand the reasons behind their 'stress eating' habits, as well as conducting more invasive health assessments (i.e., blood collections) on individuals' levels of cholesterol and triglycerides.

Further, it would be valuable to examine additional moderators in the job stressor-employee health relationship, such as PE fit, perceived organizational support, organizational commitment, or leader member exchange (LMX), which may differ for males and females, older versus younger employees, or individuals in different occupations. It is possible that another organizational construct, such as perceived organizational support, would buffer and/or mediate the relationship between job stressors and employee health better than organizational justice if one considers that supervisors are only in control of their own actions and their treatment of their employees and have little control over company-wide policies and procedures that may still negatively impact employees despite their best intentions. And with regards to the dynamics between supervisors and their subordinates, perhaps it may be more fruitful to explore aspects of interpersonal justice (Bies \& Moag, 1986; Colquitt, 2001) or LMX to truly capture the essence of the interpersonal dynamic between the employee and his supervisor, as well as supervisors' influence on the employee.

Lastly, in light of recent meta-analyses on the effect of high stress jobs on leisure-time physical inactivity (Fransson et al., 2012) and its impact on health outcomes (Robbins et al., 2011), future research should continue to investigate these relationships to expand our understanding of the more distal, yet worrisome 
physical health problems, such as Type 2 diabetes, obesity and cardiovascular disease (Borger et al., 2006; Goetzel et al., 2001). Not only are obesity and physical inactivity a growing public health concern in that they are leading risk factors for morbidity and mortality, but they are also risk factors for increased health care expenditures and absenteeism among employees (Atlantis et al., 2006; Goetzel et al., 2001), which should be of high concern to all organizational leaders.

\section{Conclusion}

Fairness perceptions are critical for organizations to attend to as research has shown a trickle down model in which fair work procedures and treating employees respectfully and honestly may have positive "spillover effects" on other aspect of their work and non-work lives (Bowen, Gilliland, \& Folger, 1999). Higher levels of justice are also valued in that organizational outcomes appear to be more controllable and predictable to employees. Studies have shown that higher levels of organizational commitment to employees and more transparent and forthcoming communication may enhance customer-oriented behaviors, which in turn influences satisfaction, retention, and even an organization's level of competitiveness (Masterson, 2001; Simons \& Roberson, 2003). Aside from focusing on justice perspectives, meta-analytic studies also suggest that risk management among people with high stress jobs should focus on glucose levels (diabetes) and lifestyle factors, as those appear to be two of the most prominent linking mechanisms between job stressors and subsequent cardiovascular disease risk factors (Nyberg et al., 2012; Nyberg et al., 2013). That said, it may be worthwhile for employers to assess the health of their organizations and then take the appropriate steps to 
incorporate better health behaviors (i.e., increase levels of physical activity or decrease occurrences of stress eating) depending on the assessment findings. In the spirit of the NIOSH Total Worker Health program (Schill \& Chosewood, 2013), this study has significant implications for researchers and practitioners alike to further expand upon these findings and implement them into organizational practice in hopes of promoting an overall paradigm shift toward a healthier workplace. This endeavor is the result of the combination of employers transforming the work environment into a more just, transparent and trustworthy place to work, starting with the dynamics between supervisors and their employees, in conjunction with the development of targeted interventions on employees' modifiable behaviors, such as engaging in physical activity and healthier eating habits.

With healthcare costs at an all time high and stress related statistics pointing to the fact that individuals nationwide are reaching a critical threshold of the amount of stress they can endure, the time has never been more appropriate for researchers and practitioners to align forces to detect the overall health of organizations and identify ways in which to make the workplace environment more enjoyable and a healthier place to be. 
Table 1. Summary of Study Hypotheses and Hypothesis Results

\begin{tabular}{cccccc}
\hline Hypothesis & Antecedent & Moderator & Mediator & Outcome & Sig Result \\
\hline $1 \mathrm{a}$ & Job Demands (JD) & & & Job Satisfaction & $*$ \\
$1 \mathrm{~b}$ & Skill Discretion (SD) & & & Job Satisfaction & $*$ \\
$1 \mathrm{c}$ & Decision Authority (DA) & & & Job Satisfaction & $*$ \\
$2 \mathrm{a}$ & Distributive Justice (DJ) & & & Job Satisfaction & $*$ \\
$2 \mathrm{~b}$ & Procedural Justice (PJ) & & & Job Satisfaction & $*$ \\
$3 \mathrm{a}$ & JD & DJ & & Job Satisfaction & - \\
$3 \mathrm{~b}$ & JD & PJ & & Job Satisfaction & - \\
$4 \mathrm{a}$ & SD & DJ & & Job Satisfaction & - \\
$4 \mathrm{~b}$ & SD & PJ & & Job Satisfaction & - \\
$5 \mathrm{a}$ & DA & DJ & & Job Satisfaction & - \\
$5 \mathrm{~b}$ & DA & PJ & & Job Satisfaction & - \\
$6 \mathrm{a}$ & JD & & & BMI & - \\
$6 \mathrm{~b}$ & SD & & & BMI & $*$ \\
$6 \mathrm{c}$ & DA & & & BMI & $*$ \\
$7 \mathrm{a}$ & DJ & & & BMI & $*$ \\
$7 \mathrm{~b}$ & PJ & & & BMI & $*$ \\
$8 \mathrm{a}$ & JD & DJ & & BMI & - \\
$8 \mathrm{~b}$ & JD & PJ & & BMI & - \\
$9 \mathrm{a}$ & SD & DJ & & BMI & - \\
$9 \mathrm{~b}$ & SD & PJ & & BMI & - \\
$10 \mathrm{a}$ & DA & DJ & & BMI & - \\
$10 \mathrm{~b}$ & DA & PJ & & BMI & - \\
$11 \mathrm{a}$ & JD & & Exercise & BMI & - \\
$11 \mathrm{~b}$ & SD & & Exercise & BMI & - \\
$11 \mathrm{c}$ & DA & & Exercise & BMI & - \\
$11 \mathrm{~d}$ & JD & & Exercise & Job Satisfaction & - \\
$11 \mathrm{e}$ & SD & & Exercise & Job Satisfaction & - \\
$11 \mathrm{f}$ & DA & & Exercise & Job Satisfaction & - \\
\hline & $\mathrm{F}$ & & & &
\end{tabular}

Note: $E A=$ exploratory analysis. ${ }^{*}=p<.05$ 
Table 1. Summary of Study Hypotheses and Hypothesis Results (continued)

\begin{tabular}{|c|c|c|c|c|c|}
\hline Hypothesis & Antecedent & Moderator & Mediator & Outcome & Sig Result \\
\hline $12 \mathrm{a}$ & JD & $\mathrm{PJ}$ & Exercise & BMI & - \\
\hline $12 b$ & JD & DJ & Exercise & BMI & - \\
\hline $13 a$ & SD & PJ & Exercise & BMI & - \\
\hline $13 b$ & SD & DJ & Exercise & BMI & - \\
\hline $14 a$ & DA & PJ & Exercise & BMI & - \\
\hline $14 \mathrm{~b}$ & DA & DJ & Exercise & BMI & - \\
\hline $15 a$ & JD & PJ & Exercise & Job Satisfaction & - \\
\hline $15 b$ & JD & DJ & Exercise & Job Satisfaction & - \\
\hline $16 a$ & SD & PJ & Exercise & Job Satisfaction & - \\
\hline $16 \mathrm{~b}$ & SD & DJ & Exercise & Job Satisfaction & - \\
\hline $17 \mathrm{a}$ & DA & PJ & Exercise & Job Satisfaction & - \\
\hline $17 \mathrm{~b}$ & DA & DJ & Exercise & Job Satisfaction & - \\
\hline EA1 & DJ & & Exercise & Job Satisfaction & - \\
\hline EA2 & PJ & & Exercise & Job Satisfaction & - \\
\hline EA3 & DJ & & Exercise & BMI & - \\
\hline EA4 & PJ & & Exercise & BMI & - \\
\hline EA5 & JD & & PJ & Job Satisfaction & $*$ \\
\hline EA6 & SD & & PJ & Job Satisfaction & $*$ \\
\hline EA7 & DA & & PJ & Job Satisfaction & $*$ \\
\hline EA8 & JD & & DJ & Job Satisfaction & $*$ \\
\hline EA9 & SD & & DJ & Job Satisfaction & * \\
\hline EA10 & DA & & DJ & Job Satisfaction & $*$ \\
\hline EA11 & JD & & PJ & BMI & - \\
\hline EA12 & SD & & PJ & BMI & - \\
\hline EA13 & DA & & PJ & BMI & - \\
\hline EA14 & JD & Exercise & & BMI & * \\
\hline EA15 & DJ & Exercise & & Job Satisfaction & $*$ \\
\hline
\end{tabular}

Note: $E A=$ exploratory analysis. ${ }^{*}=p<.05$ 
Table 2. Means, Standard Deviations, Intercorrelations, and Alpha Reliability Statistics for Study Variables

\begin{tabular}{|c|c|c|c|c|c|c|c|c|c|c|c|c|c|}
\hline Variable & Mean & SD & 1 & 2 & 3 & 4 & 5 & 6 & 7 & 8 & 9 & 10 & 11 \\
\hline 1. Gender & 1.10 & .31 & - & & & & & & & & & & \\
\hline 2. Age & 44.48 & 9.56 & .03 & - & & & & & & & & & \\
\hline 3. Education & 3.72 & .74 & $.11^{*}$ & .05 & - & & & & & & & & \\
\hline 4. Race & 1.76 & 1.66 & .06 & -.07 & .04 & - & & & & & & & \\
\hline 5. Relationship Status & 1.87 & 1.41 & $.26^{* *}$ & $-.16^{* *}$ & .10 & .08 & - & & & & & & \\
\hline 6. Hrs Worked Per Day & 8.58 & 1.01 & -.06 & -.01 & .07 & .02 & $-.13^{*}$ & - & & & & & \\
\hline 7. Hrs Worked Per Wk & 40.84 & 8.48 & $-.11^{*}$ & -.02 & .03 & -.04 & -.08 & $.33^{* *}$ & - & & & & \\
\hline 8. Years at Current Job & 11.06 & 7.96 & .07 & $.50^{* *}$ & -.08 & -.08 & -.09 & -.02 & .01 & - & & & \\
\hline 9. Organizational Role & 2.74 & 0.75 & .05 & -.06 & -.03 & .03 & $.12^{*}$ & $-.36^{* *}$ & $-.13^{*}$ & $-.15^{* *}$ & - & & \\
\hline 10. Job Demands & 2.99 & 0.67 & .00 & $.11^{*}$ & .04 & .06 & $-.15^{* *}$ & $.20^{* *}$ & $.12^{*}$ & $.21^{* *}$ & $-.24^{* *}$ & $(.72)$ & \\
\hline 11. Skill Discretion & 3.35 & 0.67 & .01 & .01 & -.03 & -.01 & -.10 & $.11^{*}$ & .01 & .09 & $-.20^{* *}$ & $.11^{*}$ & $(.76)$ \\
\hline 12. Decision Authority & 3.33 & 0.99 & -.07 & $.12^{*}$ & .07 & -.04 & $-.15^{* *}$ & .06 & .03 & $.19^{* *}$ & $-.23^{* *}$ & .09 & $.56^{* *}$ \\
\hline 13. Exercise & 2.65 & 1.58 & -.05 & $-.11^{*}$ & $.13^{*}$ & .06 & .02 & -.02 & -.05 & -.10 & .03 & .09 & -.00 \\
\hline 14. Distributive Justice & 3.08 & 1.00 & -.04 & -.08 & -.09 & -.10 & .04 & $-.12^{*}$ & -.09 & -.08 & .02 & $-.24^{* *}$ & $.30^{* *}$ \\
\hline 15. Procedural Justice & 3.32 & 1.36 & $-.11^{*}$ & .04 & .01 & $-.15^{* *}$ & -.08 & .07 & -.04 & -.03 & -.05 & $-.11^{*}$ & $.29^{* *}$ \\
\hline 16. Job Satisfaction & 3.89 & 0.79 & -.04 & -.05 & -.09 & -.04 & .00 & -.07 & .07 & -.00 & .03 & $-.13^{*}$ & $.39^{* *}$ \\
\hline 17. Body Mass Index & 3.45 & 1.16 & -.02 & .05 & $-.27^{* *}$ & .04 & -.06 & .04 & -.04 & .06 & -.02 & .07 & -.07 \\
\hline
\end{tabular}

Note. Values in parenthesis are Alpha statistics. ${ }^{*} p<.05 .{ }^{* *} p<.01$. For gender, male $=1$, female $=2$. For education, $1=$ some high school, $2=$ high school graduate, $3=$ some college, $4=$ college graduate, $5=$ attended graduate school. For race, $1=$ Caucasian, $2=$ African American, $3=$ American Indian, $4=$ Asian, $5=$ Native Hawaiian, $6=$ More than one race, $7=$ Other. For relationship status, $1=$ married, $2=$ divorced, $3=$ widowed, $4=\operatorname{living}$ $\mathrm{w} /$ significant other, $5=$ never married. For organizational role, $1=$ supervisor, $2=$ crew leader, $3=$ crewmember, $4=$ other. For exercise $(4-i t e m)$ scale, range $=0$ to 7 days. For BMI, $1=$ lean, $2=$ medium, $3=$ high, $4=$ obesity class $1,5=$ obesity class 2 . 
Table 2. Means, Standard Deviations, Intercorrelations, and Alpha Reliability Statistics for Study Variables (continued)

\begin{tabular}{lcccccc}
\hline Variable & 12 & 13 & 14 & 15 & 16 & 17 \\
\hline 12. Decision Authority & $(.88)$ & & & & & \\
13. Exercise & .05 & $(.82)$ & & & & \\
14. Distributive Justice & $.33^{* *}$ & .05 & $(.93)$ & & & \\
15. Procedural Justice & $.31^{* *}$ & .03 & $.43^{* *}$ & $(.95)$ & & \\
16. Job Satisfaction & $.32^{* *}$ & -.05 & $.45^{* *}$ & $.35^{* *}$ & $(.87)$ & \\
17. Body Mass Index & $-.17^{* *}$ & $-.19^{* *}$ & -.07 & -.08 & -.06 & - \\
\hline
\end{tabular}

Note. Values in parenthesis are Alpha statistics. ${ }^{*} p<.05 .{ }^{* *} p<.01$. For gender, male $=1$, female $=2$. For education, $1=$ some high school, $2=$ high school graduate, $3=$ some college, $4=$ college graduate, $5=$ attended graduate school. For race, $1=$ Caucasian, $2=$ African American, $3=$ American Indian, $4=$ Asian, $5=$ Native Hawaiian, $6=$ More than one race, $7=$ Other. For relationship status, $1=$ married, $2=$ divorced, $3=$ widowed, $4=$ living w/ significant other, $5=$ never married. For organizational role, $1=$ supervisor, $2=$ crew leader, $3=$ crewmember, $4=$ other. For exercise $(4-i t e m)$ scale, range $=0$ to 7 days. For BMI, $1=$ lean, $2=$ medium, $3=$ high, $4=$ obesity class $1,5=$ obesity class 2 . 
Table 3. Main Effects of Job Stressors on Job Satisfaction (H1a-H1c)

\begin{tabular}{|c|c|c|c|c|c|}
\hline Variable & $R^{2}$ & $\Delta R^{2}$ & $F$ for $\Delta R^{2}$ & $\beta$ & $t$ \\
\hline \multicolumn{6}{|c|}{ H1a: Job Satisfaction Regressed on Job Demands } \\
\hline Step 1: Control & .01 & .01 & 1.66 & & \\
\hline Organization & & & & -.07 & -1.29 \\
\hline \multicolumn{6}{|l|}{ Step 2: Predictor } \\
\hline Job Demands & .02 & $.01^{*}$ & 3.37 & $-.12^{*}$ & -2.25 \\
\hline \multicolumn{6}{|c|}{ H1b: Job Satisfaction Regressed on Skill Discretion } \\
\hline Step 1: Control & .01 & .01 & 1.66 & & \\
\hline Organization & & & & -.07 & -1.29 \\
\hline \multicolumn{6}{|l|}{ Step 2: Predictor } \\
\hline Skill Discretion & .16 & $.16^{* * *}$ & 33.26 & $.40^{* * *}$ & 8.04 \\
\hline \multicolumn{6}{|c|}{ H1c: Job Satisfaction Regressed on Decision Authority } \\
\hline Step 1: Control & .00 & .00 & 1.53 & & \\
\hline Organization & & & & -.07 & -1.24 \\
\hline \multicolumn{6}{|l|}{ Step 2: Predictor } \\
\hline Decision Authority & .11 & $.11^{* * *}$ & 22.03 & $.33^{* * *}$ & 6.51 \\
\hline
\end{tabular}

Note. ${ }^{*} \mathrm{p}<.05{ }^{* *} \mathrm{p}<.01,{ }^{* * *} \mathrm{p}<.001$. Three separate regression analyses were run to test Hypotheses 1a-1c; however, for the sake of brevity, results were included in the same table. The outcome variable was job satisfaction; the predictors were job demands, skill discretion, and decision authority; and the control variable was organization. 
Table 4. Main Effects of Organizational Justice on Job Satisfaction (H2a-H2b)

\begin{tabular}{lccccc}
\hline \multicolumn{1}{c}{ Variable } & $R^{2}$ & $\Delta R^{2}$ & $F$ for $\Delta R^{2}$ & $\beta$ & $t$ \\
\hline $\begin{array}{l}\text { H2a: Job Satisfaction Regressed on Distributive Justice } \\
\text { Step 1: Control }\end{array}$ & .00 & .00 & 1.44 & & \\
$\quad$ Organization & & & & -.07 & -1.20 \\
$\begin{array}{l}\text { Step 2: Predictor } \\
\quad \text { Distributive Justice }\end{array}$ & .20 & $.20^{* * *}$ & 43.79 & $.45^{* * *}$ & 9.26 \\
$\begin{array}{l}\text { H2b: Job Satisfaction Regressed on Procedural Justice } \\
\text { Step 1: Control } \\
\quad \text { Organization }\end{array}$ & .01 & .01 & 1.57 & & \\
$\begin{array}{l}\text { Step 2: Predictor } \\
\text { Procedural Justice }\end{array}$ & .14 & $.13^{* * *}$ & 26.90 & $.37^{* * *}$ & 7.21 \\
\hline
\end{tabular}

Note. ${ }^{*} \mathrm{p}<.05,{ }^{* *} \mathrm{p}<.01,{ }^{* * *} \mathrm{p}<.001$. Two separate regression analyses were run to test Hypotheses $2 \mathrm{a}-2 \mathrm{~b}$; however, for the sake of brevity, results were included in the same table. The outcome variable was job satisfaction; the predictors were distributive and procedural justice; and the control variable was organization. 
Table 5. Interaction of Distributive Justice and Job Demands on Job Satisfaction ( $\mathrm{H} 3 a$ )

\begin{tabular}{lllllc}
\hline \multicolumn{1}{c}{ Variable } & $R^{2}$ & $\Delta R^{2}$ & $F$ for $\Delta R^{2}$ & $\beta$ & $t$ \\
\hline Step 1: Control & .00 & .00 & 1.44 & & \\
$\quad$ Organization & & & & -.06 & -1.20 \\
Step 2: Predictors & .20 & $.20^{* * *}$ & 29.17 & & \\
$\quad$ Distributive Justice & & & & $.44^{* * *}$ & 8.89 \\
$\quad$ Job Demands & & & & -.02 & -.39 \\
$\begin{array}{l}\text { Step 3: Interaction Term } \\
\quad \text { DJ x Job Demands }\end{array}$ & .21 & .00 & 22.05 & & \\
\hline
\end{tabular}

Note. ${ }^{*} \mathrm{p}<.05,{ }^{* *} \mathrm{p}<.01,{ }^{* * *} \mathrm{p}<.001$. The outcome variable was job satisfaction. The control variable (organization) was entered into Step 1; the mean-centered independent variable (job demands) and moderator (distributive justice) were entered into Step 2; and the interaction term was entered into Step 3. 
Table 6. Interaction of Procedural Justice and Job Demands on Job Satisfaction (H3b)

\begin{tabular}{lccccc}
\hline \multicolumn{1}{c}{ Variable } & $R^{2}$ & $\Delta R^{2}$ & $F$ for $\Delta R^{2}$ & $\beta$ & $t$ \\
\hline Step 1: Control & .01 & .01 & 1.57 & & \\
$\quad$ Organization & & & & -.07 & -1.25 \\
Step 2: Predictors & .14 & $.14^{* * *}$ & 18.61 & & \\
$\quad$ Procedural Justice & & & & $.36^{* * *}$ & 7.00 \\
$\quad$ Job Demands & & & & -.07 & -1.37 \\
$\quad$ Step 3: Interaction Term & .15 & .01 & 14.47 & & \\
$\quad$ PJ x Job Demands & & & & .07 & 1.39 \\
\hline
\end{tabular}

Note. ${ }^{*} \mathrm{p}<.05,{ }^{* *} \mathrm{p}<.01,{ }^{* * *} \mathrm{p}<.001$. The outcome variable was job satisfaction. The control variable (organization) was entered into Step 1; the mean-centered independent variable (job demands) and moderator (procedural justice) were entered into Step 2; and the interaction term was entered into Step 3. 
Table 7. Interaction of Distributive Justice and Skill Discretion on Job Satisfaction (H4a)

\begin{tabular}{lllllc}
\hline \multicolumn{1}{c}{ Variable } & $R^{2}$ & $\Delta R^{2}$ & $F$ for $\Delta R^{2}$ & $\beta$ & $t$ \\
\hline $\begin{array}{l}\text { Step 1: Control } \\
\quad \text { Organization }\end{array}$ & .00 & .00 & 1.44 & & \\
$\quad$ Step 2: Predictors & .28 & $.27^{* * *}$ & 43.97 & & -1.20 \\
$\quad$ Distributive Justice & & & & $.34^{* * *}$ & 7.41 \\
$\quad$ Skill Discretion & & & & $.29^{* * *}$ & 5.96 \\
$\quad \begin{array}{l}\text { Step 3: Interaction Term } \\
\quad \text { DJ x Skill Discretion }\end{array}$ & .28 & .00 & 33.37 & & \\
\hline
\end{tabular}

Note. ${ }^{*} \mathrm{p}<.05,{ }^{* *} \mathrm{p}<.01,{ }^{* * *} \mathrm{p}<.001$. The outcome variable was job satisfaction. The control variable (organization) was entered into Step 1; the mean-centered independent variable (skill discretion) and moderator (distributive justice) were entered into Step 2; and the interaction term was entered into Step 3. 
Table 8. Interaction of Procedural Justice and Skill Discretion on Job Satisfaction (H4b)

\begin{tabular}{lllllc}
\hline \multicolumn{1}{c}{ Variable } & $R^{2}$ & $\Delta R^{2}$ & $F$ for $\Delta R^{2}$ & $\beta$ & $t$ \\
\hline $\begin{array}{l}\text { Step 1: Control } \\
\quad \text { Organization }\end{array}$ & .01 & .01 & 1.57 & & \\
$\quad$ Step 2: Predictors & .23 & $.23^{* * *}$ & 34.11 & & -1.25 \\
$\quad$ Procedural Justice & & & & $.28^{* * *}$ & 5.62 \\
$\quad$ Skill Discretion & & & & .32 & 6.48 \\
$\quad \begin{array}{l}\text { Step 3: Interaction Term } \\
\quad \text { PJ x Skill Discretion }\end{array}$ & .23 & .00 & 25.63 & & \\
\hline
\end{tabular}

Note. ${ }^{*} \mathrm{p}<.05,{ }^{* *} \mathrm{p}<.01,{ }^{* * *} \mathrm{p}<.001$. The outcome variable was job satisfaction. The control variable (organization) was entered into Step 1; the mean-centered independent variable (skill discretion) and moderator (procedural justice) were entered into Step 2; and the interaction term was entered into Step 3. 
Table 9. Interaction of Distributive Justice and Decision Authority on Job Satisfaction (H5a)

\begin{tabular}{|c|c|c|c|c|c|}
\hline Variable & $R^{2}$ & $\Delta R^{2}$ & $F$ for $\Delta R^{2}$ & $\beta$ & $t$ \\
\hline Step 1: Control & .00 & .00 & 1.31 & & \\
\hline Organization & & & & -.06 & -1.15 \\
\hline Step 2: Predictors & .24 & $.23^{* * *}$ & 35.46 & & \\
\hline Distributive Justice & & & & $.36^{* * *}$ & 7.44 \\
\hline Decision Authority & & & & $.21^{* * *}$ & 4.03 \\
\hline Step 3: Interaction Term & .24 & .01 & 27.27 & & \\
\hline $\begin{array}{l}\text { DJ x Decision } \\
\text { Authority }\end{array}$ & & & & -.07 & -1.51 \\
\hline
\end{tabular}

Note. ${ }^{*} \mathrm{p}<.05,{ }^{* *} \mathrm{p}<.01,{ }^{* * *} \mathrm{p}<.001$. The outcome variable was job satisfaction. The control variable (organization) was entered into Step 1; the mean-centered independent variable (decision authority) and moderator (distributive justice) were entered into Step 2; and the interaction term was entered into Step 3. 
Table 10. Interaction of Procedural Justice and Decision Authority on Job Satisfaction (H5b)

\begin{tabular}{lccccc}
\hline \multicolumn{1}{c}{ Variable } & $R^{2}$ & $\Delta R^{2}$ & $F$ for $\Delta R^{2}$ & $\beta$ & $t$ \\
\hline Step 1: Control & .00 & .00 & 1.44 & & \\
$\quad$ Organization & & & & -.07 & -1.20 \\
Step 2: Predictors & $.19^{* * *}$ & $.19^{* * *}$ & 26.45 & & \\
$\quad$ Procedural Justice & & & & $.30^{* * *}$ & 7.00 \\
$\quad$ Decision Authority & & & & $.25^{* * *}$ & 4.84 \\
Step 3: Interaction Term & .19 & .00 & 19.96 & & \\
$\quad$ PJ x Decision Authority & & & & -.04 & -.75 \\
\hline
\end{tabular}

Note. ${ }^{*} \mathrm{p}<.05,{ }^{* *} \mathrm{p}<.01,{ }^{* * *} \mathrm{p}<.001$. The outcome variable was job satisfaction. The control variable (organization) was entered into Step 1; the mean-centered independent variable (decision authority) and moderator (procedural justice) were entered into Step 2; and the interaction term was entered into Step 3. 
Table 11. Main Effects of Job Stressors on BMI (H6a-H6c)

\begin{tabular}{|c|c|c|c|c|c|}
\hline Variable & $R^{2}$ & $\Delta R^{2}$ & $F$ for $\Delta R^{2}$ & $\beta$ & $t$ \\
\hline \multicolumn{6}{|c|}{ H6a: BMI Regressed on Job Demands } \\
\hline Step 1: Controls & .07 & .07 & 8.37 & & \\
\hline Organization & & & & .01 & .14 \\
\hline Education & & & & $-.23^{* * *}$ & -4.13 \\
\hline Active at Work & & & & $-.14^{* *}$ & -2.65 \\
\hline \multicolumn{6}{|l|}{ Step 2: Predictor } \\
\hline Job Demands & .08 & .00 & 6.59 & .06 & 1.10 \\
\hline \multicolumn{6}{|c|}{ H6b: BMI Regressed on Skill Discretion } \\
\hline Step 1: Controls & .07 & .07 & 8.37 & & \\
\hline Organization & & & & .01 & .14 \\
\hline Education & & & & $-.23^{* * *}$ & -4.13 \\
\hline Active at Work & & & & $-.14^{* *}$ & -2.65 \\
\hline \multicolumn{6}{|l|}{ Step 2: Predictor } \\
\hline Skill Discretion & .09 & $.01^{*}$ & 7.40 & $-.11^{*}$ & -2.05 \\
\hline \multicolumn{6}{|c|}{ H6c: BMI Regressed on Decision Authority } \\
\hline Step 1: Controls & .08 & .08 & 8.45 & & \\
\hline Organization & & & & .01 & .13 \\
\hline Education & & & & $-.24^{* * *}$ & -4.14 \\
\hline Active at Work & & & & $-.15^{* *}$ & -2.66 \\
\hline \multicolumn{6}{|l|}{ Step 2: Predictor } \\
\hline Decision Authority & .11 & $.03^{* *}$ & 9.30 & $-.18^{* *}$ & -3.32 \\
\hline
\end{tabular}

Note. ${ }^{*} \mathrm{p}<.05{ }^{* *} \mathrm{p}<.01,{ }^{* * *} \mathrm{p}<.001$. Three separate regression analyses were run to test Hypotheses 6a-6c; however, for the sake of brevity, results were included in the same table. The outcome variable was BMI; the predictors were job demands, skill discretion, and decision authority; and the control variables were organization, education, and 'active at work'. 
Table 12. Main Effect of Organizational Justice on BMI (H7a-H7b)

\begin{tabular}{|c|c|c|c|c|c|}
\hline Variable & $R^{2}$ & $\Delta R^{2}$ & $F$ for $\Delta R^{2}$ & $\beta$ & $t$ \\
\hline \multicolumn{6}{|c|}{ H7a: BMI Regressed on Distributive Justice } \\
\hline Step 1: Control & .07 & .07 & 8.00 & & \\
\hline Organization & & & & .01 & .15 \\
\hline Education & & & & -.24 & -4.16 \\
\hline Active at Work & & & & -.13 & -2.36 \\
\hline \multicolumn{6}{|l|}{ Step 2: Predictor } \\
\hline Distributive Justice & .09 & $.02^{*}$ & 7.39 & $-.13^{*}$ & -2.29 \\
\hline \multicolumn{6}{|c|}{ H7b: BMI Regressed on Procedural Justice } \\
\hline Step 1: Control & .07 & .07 & 8.27 & & \\
\hline Organization & & & & .00 & .06 \\
\hline Education & & & & $-.23^{* * *}$ & -4.11 \\
\hline Active at Work & & & & $-.14^{* *}$ & -2.60 \\
\hline \multicolumn{6}{|l|}{ Step 2: Predictor } \\
\hline Procedural Justice & .09 & $.02 *$ & 7.59 & $-.13^{*}$ & -2.28 \\
\hline
\end{tabular}


Table 13. Interaction of Distributive Justice and Job Demands on BMI (H8a)

\begin{tabular}{lccccc}
\hline \multicolumn{1}{c}{ Variable } & $R^{2}$ & $\Delta R^{2}$ & $F$ for $\Delta R^{2}$ & $\beta$ & $t$ \\
\hline Step 1: Control & .07 & .07 & 8.00 & & \\
$\quad$ Organization & & & & .01 & .15 \\
$\quad$ Education & & & & -.24 & -4.16 \\
$\quad$ Active at Work & & & & -.13 & -2.36 \\
Step 2: Predictors & .09 & .02 & 5.91 & & \\
$\quad$ Distributive Justice & & & & -.12 & -2.14 \\
$\quad$ Job Demands & & & & .02 & .33 \\
Step 3: Interaction Term & .09 & .00 & 5.10 & & \\
$\quad$ DJ x Job Demands & & & & .06 & 1.00 \\
\hline
\end{tabular}

Note. ${ }^{*} \mathrm{p}<.05,{ }^{* *} \mathrm{p}<.01,{ }^{* * *} \mathrm{p}<.001$. The outcome variable was BMI. The control variables (organization, education, and active at work) were entered into Step 1; the mean-centered independent variable (job demands) and moderator (distributive justice) were entered into Step 2; and the interaction term was entered into Step 3. 
Table 14. Interaction of Procedural Justice and Job Demands on BMI (H8b)

\begin{tabular}{lccccc}
\hline \multicolumn{1}{c}{ Variable } & $R^{2}$ & $\Delta R^{2}$ & $F$ for $\Delta R^{2}$ & $\beta$ & $t$ \\
\hline Step 1: Control & .07 & .07 & 8.27 & & \\
$\quad$ Organization & & & & .00 & .06 \\
$\quad$ Education & & & & -.23 & -4.11 \\
$\quad$ Active at Work & & & & -.14 & -2.6 \\
Step 2: Predictors & .09 & .02 & 6.22 & & \\
$\quad$ Procedural Justice & & & & $-.12^{*}$ & -2.17 \\
$\quad$ Job Demands & & & & .05 & .88 \\
Step 3: Interaction Term & .10 & .00 & 5.42 & & \\
$\quad$ PJ x Job Demands & & & & .07 & 1.17 \\
\hline
\end{tabular}

Note. ${ }^{*} \mathrm{p}<.05,{ }^{* *} \mathrm{p}<.01,{ }^{* * *} \mathrm{p}<.001$. The outcome variable was BMI. The control variables (organization, education, and active at work) were entered into Step 1; the mean-centered independent variable (job demands) and moderator (procedural justice) were entered into Step 2; and the interaction term was entered into Step 3. 
Table 15. Interaction of Distributive Justice and Skill Discretion on BMI (H9a)

\begin{tabular}{lccccc}
\hline \multicolumn{1}{c}{ Variable } & $R^{2}$ & $\Delta R^{2}$ & $F$ for $\Delta R^{2}$ & $\beta$ & $t$ \\
\hline Step 1: Control & .07 & .07 & 8.00 & & \\
$\quad$ Organization & & & & .01 & .15 \\
$\quad$ Education & & & & -.24 & -4.16 \\
$\quad$ Active at Work & & & & -.13 & -2.36 \\
Step 2: Predictors & .09 & $.02^{*}$ & 6.30 & & \\
$\quad$ Distributive Justice & & & & -.10 & -1.78 \\
$\quad$ Skill Discretion & & & & -.08 & -1.36 \\
Step 3: Interaction Term & .09 & .00 & 5.31 & & \\
$\quad$ DJ x Skill Discretion & & & & -.04 & -.65 \\
\hline
\end{tabular}

Note. ${ }^{*} \mathrm{p}<.05,{ }^{* *} \mathrm{p}<.01,{ }^{* * *} \mathrm{p}<.001$. The outcome variable was BMI. The control variables (organization, education, and active at work) were entered into Step 1; the mean-centered independent variable (skill discretion) and moderator (distributive justice) were entered into Step 2; and the interaction term was entered into Step 3. 
Table 16. Interaction of Procedural Justice and Skill Discretion on BMI (H9b)

\begin{tabular}{llcccc}
\hline \multicolumn{1}{c}{ Variable } & $R^{2}$ & $\Delta R^{2}$ & $F$ for $\Delta R^{2}$ & $\beta$ & $t$ \\
\hline Step 1: Control & .07 & .07 & 8.27 & & \\
$\quad$ Organization & & & & .00 & .06 \\
$\quad$ Education & & & & -.23 & -4.11 \\
$\quad$ Active at Work & & & & -.14 & -2.6 \\
Step 2: Predictors & .10 & $.02^{*}$ & 6.51 & & \\
$\quad$ Procedural Justice & & & & -.11 & -1.85 \\
$\quad$ Skill Discretion & & & & -.08 & -1.45 \\
Step 3: Interaction Term & .10 & .01 & 5.78 & & \\
$\quad$ PJ x Skill Discretion & & & & -.08 & -1.42 \\
\hline
\end{tabular}

Note. ${ }^{*} \mathrm{p}<.05,{ }^{* *} \mathrm{p}<.01,{ }^{* * *} \mathrm{p}<.001$. The outcome variable was BMI. The control variables (organization, education, and active at work) were entered into Step 1; the mean-centered independent variable (skill discretion) and moderator (procedural justice) were entered into Step 2; and the interaction term was entered into Step 3. 
Table 17. Interaction of Distributive Justice and Decision Authority on BMI (H10a)

\begin{tabular}{lllllc}
\hline \multicolumn{1}{c}{ Variable } & $R^{2}$ & $\Delta R^{2}$ & $F$ for $\Delta R^{2}$ & $\beta$ & $t$ \\
\hline Step 1: Control & .07 & .07 & 8.01 & & \\
$\quad$ Organization & & & & .01 & .14 \\
$\quad$ Education & & & & -.24 & -4.17 \\
$\quad$ Active at Work & & & & -.13 & -2.37 \\
Step 2: Predictors & .11 & $.04^{* *}$ & 7.53 & & \\
$\quad$ Distributive Justice & & & & -.07 & -1.25 \\
$\quad$ Decision Authority & & & & -.16 & -2.72 \\
Step 3: Interaction Term & .12 & .01 & 6.72 & & \\
DJ x Decision Authority & & & & .09 & 1.58 \\
\hline
\end{tabular}

Note. ${ }^{*} \mathrm{p}<.05,{ }^{* *} \mathrm{p}<.01,{ }^{* * *} \mathrm{p}<.001$. The outcome variable was BMI. The control variables (organization, education, and active at work) were entered into Step 1; the mean-centered independent variable (decision authority) and moderator (distributive justice) were entered into Step 2; and the interaction term was entered into Step 3. 
Table 18. Interaction of Procedural Justice and Decision Authority on BMI (H10b)

\begin{tabular}{llllll}
\hline \multicolumn{1}{c}{ Variable } & $R^{2}$ & $\Delta R^{2}$ & $F$ for $\Delta R^{2}$ & $\beta$ & $t$ \\
\hline Step 1: Control & .08 & .08 & 8.35 & & \\
$\quad$ Organization & & & & .00 & .04 \\
$\quad$ Education & & & & -.24 & -4.13 \\
$\quad$ Active at Work & & & & -.14 & -2.62 \\
Step 2: Predictors & .11 & $.04^{* *}$ & 7.67 & & \\
$\quad$ Procedural Justice & & & & -.08 & -1.39 \\
$\quad$ Skill Discretion & & & & -.16 & -2.71 \\
Step 3: Interaction Term & .11 & .00 & 6.38 & & \\
$\quad$ PJ x Skill Discretion & & & & -.02 & -.26 \\
\hline
\end{tabular}

Note. ${ }^{*} \mathrm{p}<.05,{ }^{* *} \mathrm{p}<.01,{ }^{* * *} \mathrm{p}<.001$. The outcome variable was BMI. The control variables (organization, education, and active at work) were entered into Step 1; the mean-centered independent variable (decision authority) and moderator (procedural justice) were entered into Step 2 ; and the interaction term was entered into Step 3. 
Table 19. Exercise as a Mediator on the Relationship between Job Stressors and BMI (H11aH11c)

\begin{tabular}{lccccc}
\hline \multicolumn{1}{c}{ Variable } & $R^{2}$ & $\Delta R^{2}$ & $F$ for $\Delta R^{2}$ & $\beta$ & $t$ \\
\hline (H11a) 1 $1^{\text {st Equation: BMI }}$ (Outcome) & Regressed on Job Demands (IV) & \\
Step 1: Controls & .07 & .07 & 8.37 & & \\
$\quad$ Organization & & & & .01 & .14 \\
$\quad$ Education & & & $-.23^{* * *}$ & -4.13 \\
$\quad$ Active at Work & & & $-.14^{* *}$ & -2.65 \\
Step 2: Predictor & .08 & .00 & 6.59 & .06 & 1.10 \\
$\quad$ Job Demands & .00
\end{tabular}

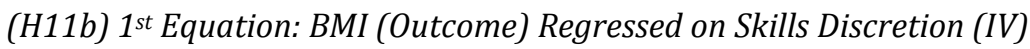
Step 1: Controls
.07
.07
8.37

Organization

Education

Active at Work

$\begin{array}{lr}.01 & .14 \\ -.23^{* * *} & -4.13 \\ -.14^{* *} & -2.65\end{array}$

Step 2: Predictor

Skill Discretion

.09

$.01^{*}$

$7.40-.11^{*}$

$-2.05$

$2^{\text {nd }}$ Equation: Exercise (Mediator) Regressed on Skill Discretion (IV)

Step 1: Controls

.01

.01

5.03

Organization

.02

.00

2.54

Step 2: Exercise on SD

.02

$-.02$

$-.28$

(H11c) 1st Equation: BMI (Outcome) Regressed on Decision Authority (IV)

\begin{tabular}{|c|c|c|c|c|c|}
\hline & & & & & \\
\hline Step 1: Controls & .08 & .08 & 8.45 & & \\
\hline Organization & & & & .01 & .13 \\
\hline Education & & & & $-.24^{* * *}$ & -4.14 \\
\hline Active at Work & & & & $-.15^{* *}$ & -2.66 \\
\hline Step 2: Predictor & & & & & \\
\hline Decision Authority & .11 & $.03^{* *}$ & 9.30 & $-.18^{* *}$ & -3.32 \\
\hline $2^{\text {nd }}$ Equation: Exercise & liator) & ressed o & ision $A$ & rity (IV) & \\
\hline Step 1: Controls & .01 & .01 & 5.03 & & \\
\hline Organization & & & & .12 & 2.24 \\
\hline Step 2: Exercise on DA & .05 & .00 & 3.92 & .03 & .60 \\
\hline
\end{tabular}

Note. ${ }^{*} \mathrm{p}<.05 .{ }^{* *} \mathrm{p}<.01,{ }^{* * *} \mathrm{p}<.001$. The outcome variable was BMI; the predictors were job demands, skill discretion, and decision authority; the mediator was exercise; and the control variables were organization, education, and 'active at work'. Regression analyses were run based on the steps outlined by Baron \& Kenny (1986) to test Hypotheses 11a-11c; because results were not significant after the $2^{\text {nd }}$ equation, equations 3 and 4 were not conducted. For the sake of brevity, results for all three hypotheses are included in the same table. 
Table 20. Exercise as a Mediator on the Relationship between Job Stressors and Job Satisfaction (H11d-H11f)

\begin{tabular}{|c|c|c|c|c|c|}
\hline Variable & $R^{2}$ & $\Delta R^{2}$ & $F$ for $\Delta R^{2}$ & $\beta$ & $t$ \\
\hline \multicolumn{6}{|c|}{ (H11d) 1 $1^{\text {st }}$ Equation: Job Satisfaction (Outcome) Regressed on Job Demands (IV) } \\
\hline Step 1: Controls & .01 & .01 & 1.66 & & \\
\hline Organization & & & & -.07 & -1.29 \\
\hline \multicolumn{6}{|l|}{ Step 2: Predictor } \\
\hline Job Demands & .02 & $.01^{*}$ & 3.37 & $-.12^{*}$ & -2.25 \\
\hline \multicolumn{6}{|c|}{$2^{\text {nd }}$ Equation: Exercise (Mediator) Regressed on Job Demands (IV) } \\
\hline Step 1: Controls & .01 & .01 & 5.03 & & \\
\hline Organization & & & & .12 & 2.24 \\
\hline Step 2: Exercise on JD & .02 & .01 & 3.73 & .08 & 1.55 \\
\hline
\end{tabular}

(H11e) 1 ${ }^{\text {st }}$ Equation: Job Satisfaction (Outcome) Regressed on Skills Discretion (IV)

Step 1: Control

$\begin{array}{lll}.01 & .01 & 1.66\end{array}$

$\begin{array}{lll}\text { Organization } & -.07 & -1.29\end{array}$

Step 2: Predictor

$\begin{array}{llllll}\text { Skill Discretion } & .16 & .16^{* * *} & 33.26 & .40^{* * *} & 8.04\end{array}$

$2^{\text {nd }}$ Equation: Exercise (Mediator) Regressed on Skill Discretion (IV)

$\begin{array}{lllllr}\text { Step 1: Controls } & .01 & .01 & 5.03 & & \\ \quad \text { Organization } & & & & .12 & 2.24 \\ \text { Step 2: Exercise on SD } & .02 & .00 & 1.52 & -.02 & .75\end{array}$

(H11f) 1 ${ }^{\text {st }}$ Equation: Job Satisfaction (Outcome) Regressed on Decision Authority (IV)

$\begin{array}{cccccc}\text { Step 1: Control } & .00 & .00 & 1.53 & & \\ \text { Organization } & & & & -.07 & -1.24\end{array}$

Step 2: Predictor

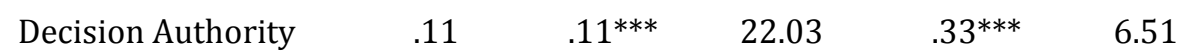

$2^{\text {nd }}$ Equation: Exercise (Mediator) Regressed on Decision Authority (IV)

$\begin{array}{llll}\text { Step 1: Controls } & .01 & .01 & 5.03\end{array}$

$\begin{array}{lll}\text { Organization } & .12 & 2.24\end{array}$

$\begin{array}{llllll}\text { Step 2: Exercise on DA } & .05 & .00 & 3.92 & .03 & .60\end{array}$

Note. ${ }^{*} \mathrm{p}<.05 .{ }^{* *} \mathrm{p}<.01,{ }^{* * *} \mathrm{p}<.001$. The outcome variable was job satisfaction; the predictors were job demands, skill discretion, and decision authority; the mediator was exercise; and the control variable was organization. Regression analyses were run based on the steps outlined by Baron \& Kenny (1986) to test Hypotheses 11d-11f; because results were not significant after the $2^{\text {nd }}$ equation, equations 3 and 4 were not conducted. For the sake of brevity, results for all three hypotheses are included in the same table. 
Figure 1. Model of hypothesized relationships and study results

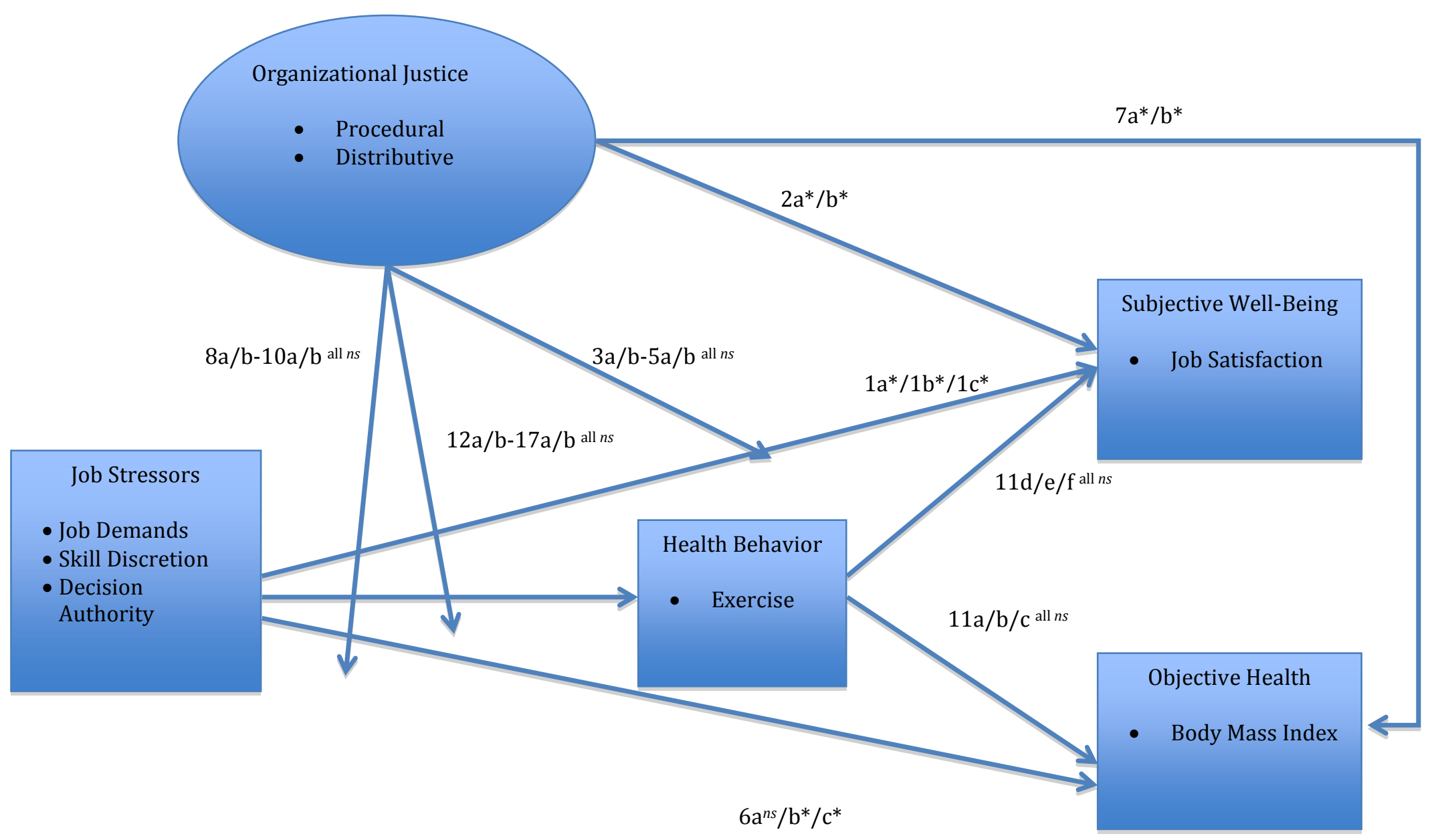

Note: ${ }^{*}=$ significant at the .05 level; $n s=$ not significant. 
Figure 2. Interaction of Exercise and Job Demands on BMI

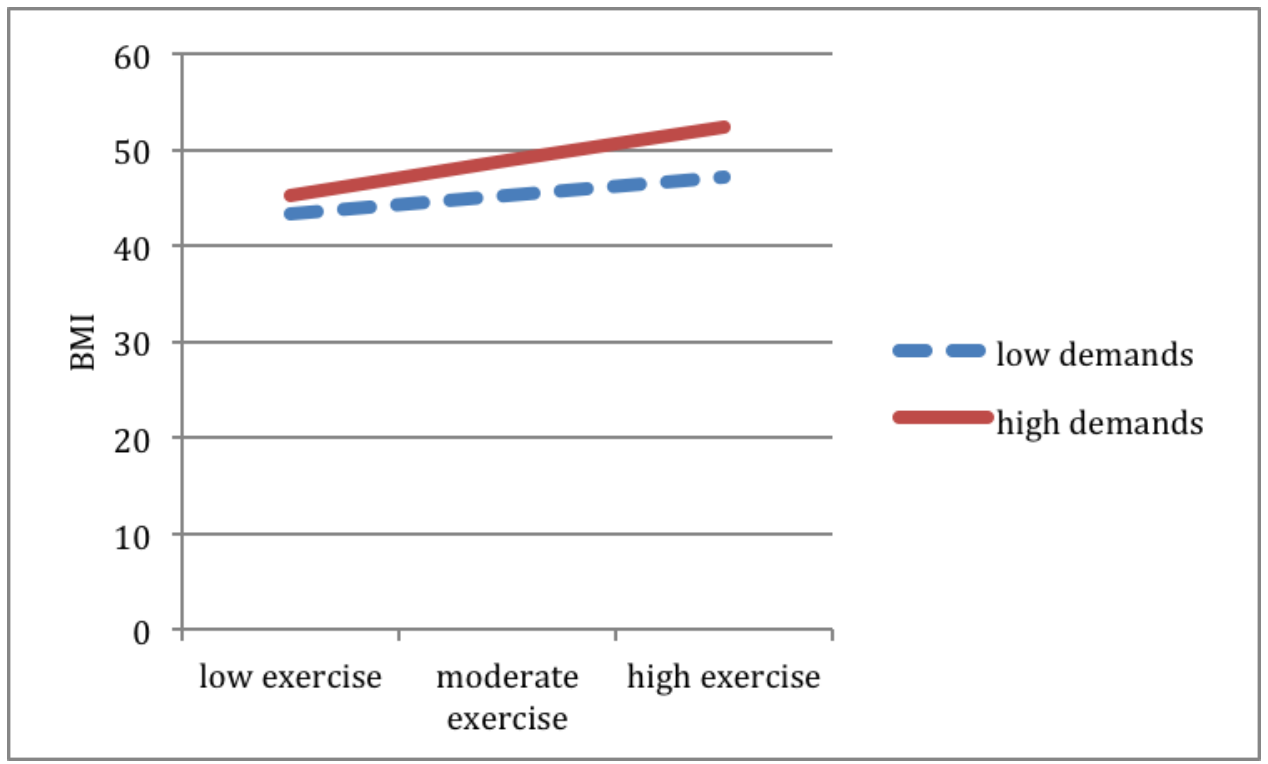


Figure 3. Interaction of Exercise and Distributive Justice on Job Satisfaction

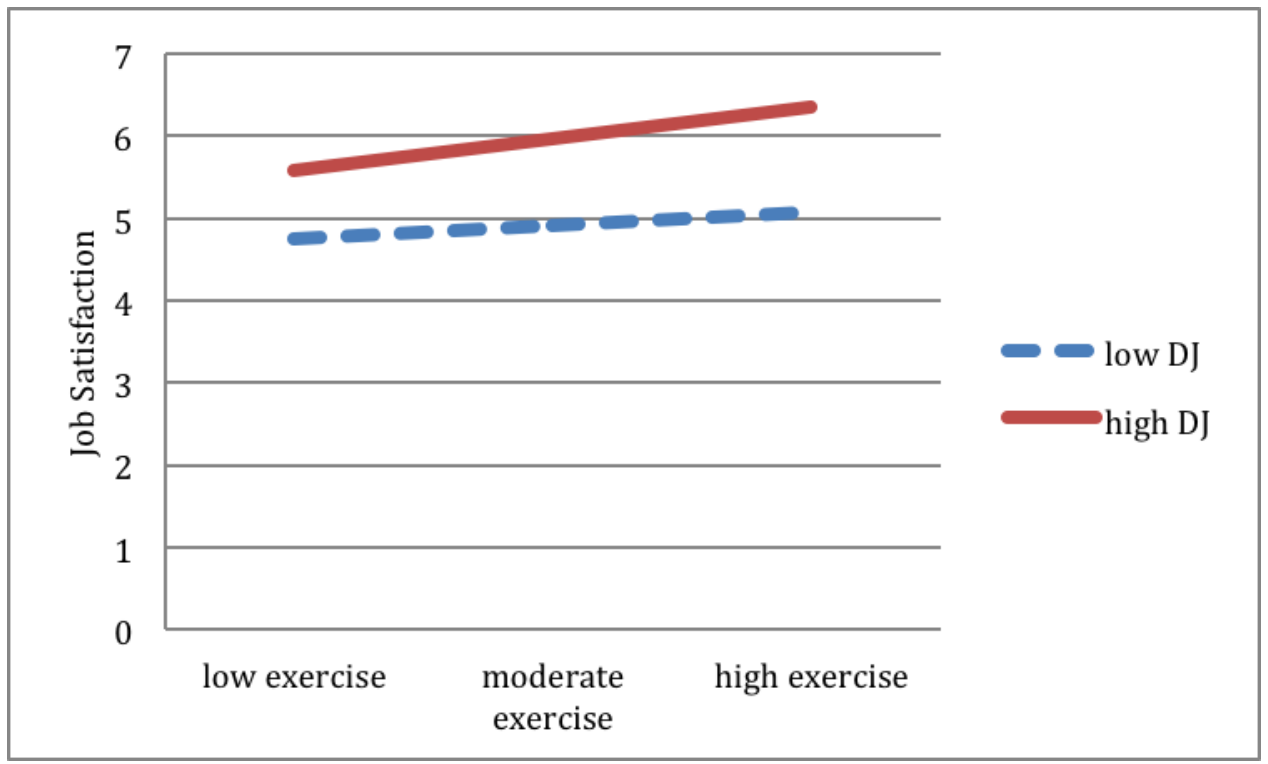




\section{References}

Adams, J. S. (1965). Inequity in social exchange. In L. Berkowitz (Ed.), Advances in Experimental Social Psychology (pp. 267-299). New York: Academic Press.

Akerstedt, T. (1997). Overview of brain metabolism and stress. In Kinney, J. M., \& Tucker, H. N. (Eds.). Lippincott-Raven: Philadelphia, pp. 131-150.

Altchiler, L., Motta, R., (1994). Effects of aerobic and nonaerobic exercise on anxiety, absenteeism, and job satisfaction. Journal of Clinical Psychology, 50, 829-840.

Ambrose, M., \& Cropanzano, R. (2003). A Longitudinal Analysis of Organizational Fairness: An Examination of Reactions to Tenure and Promotion Decisions. Journal of Applied Psychology, 88, 266-275.

American Psychological Association. (2007). Stress a major health problem in the U.S., warns APA. Retrieved June 15, 2013, from http://www.apa.org/news/press/releases/2007/10/stress.aspx American Psychological Association. (2007). Overwhelmed by workplace stress?

You're not alone. Retrieved April 12, 2014 from http://www.apa.org/helpcenter/work-stress.aspx

American Psychological Association. (2009). Fact sheet by the numbers. Retrieved June 15, 2013 from http://www.apa.org/practice/programs/workplace/phwp-fact-sheet.pdf American Psychological Association. (2013). A stress snapshot report. Retrieved April 12, 2014 from 
http://www.apa.org/news/press/releases/stress/2013/snapshot.aspx\#

Artazcoz, L., Borrell, C., Cortes, I., Escriba-Aguir, V., \& Cascant, L. (2005).

Occupational epidemiology and work related inequalities in health: a gender perspective for two complementary approaches to work and health research. Journal of Epidemiology Community Health, 61, 39-45.

Atlantis, E., Chow, C., Kirby, A., \& Fiatarone Singh, M. A. (2006). Worksite intervention effects on physical health: A randomized controlled trial. Health Promotion International, 21, 191-200.

Baron, R. M., \& Kenny, D. A. (1986). The moderator-mediator variable distinction in social psychological research: Conceptual, strategic, and statistical considerations. Journal of Personality and Social Psychology, 51, 1173-1182.

Baumeister, R. F., Bratslavsky, E., Muraven, M., \& Tice, D. M. (1998). Ego depletion: is the active self a limited resource? Journal of Personality and Social Psychology, 74(5), 1252.

Beck, A.T. (1972). Measuring depression: The depression inventory. In T.A. Williams, M.M. Katz, and J.A. Shields (Eds.), Recent advances in the psychobiology of the depressive illnesses (pp. 299-302). U.S. Government Printing Office, Washington, DC.

Beehr, T. A. \& Glazer, S. (2005). Organizational role stress. In J. Barling, E. K. Kelloway, \& M. R. Frone (Eds.), Handbook of work stress (pp. 7-33). Thousand Oaks, CA: Sage Publications.

Belkic, K. L., Landsbergis, P. A., Schall, P. L., \& Baker, D. (2004). Is job strain a major source of cardiovascular disease risk? Scandinavian Journal of Work, 
Environment and Health, 30, 85-128.

Bellavia, G. M., \& Frone, M. R. (2005). Work-family conflict. In J. Barling, E. K. Kelloway, \& M. R. Frone (Eds.), Handbook of work stress (pp. 113-147). Thousand Oaks, CA: Sage Publications.

Berset, M., Semmer, N. K., Elfering, A., Jacobshagen, N., \& Meier, L. L. (2011). Does stress at work make you gain weight? A two-year longitudinal study. Scandinavian Journal of Work Environment and Health, 37, 45-53.

Bhagat, R. S., McQuaid, S. J., Lindholm, H., \& Sergovis, J. (1985). Total life stress: A multimethod validation of the construct and its effects on organizationally valued outcomes and withdrawal behaviors. Journal of Applied Psychology, $70,202-214$

Bies, R.J. \& Moag, J.F. (1986). Interactional justice: Communication criteria of fairness. In Lewicki, R.J., Sheppard, B.H. and Bazerman, M.H. (Eds.), Research on Negotiation in Organizations (Vol. 1, pp. 43-55). Greenwich, CT: JAI Press. Blau, P. M. (1964). Exchange and power in social life. New York: Wiley.

Borger, C., Smith, S., Truffer, C., Keehan, S., Sisko, A., Poisal, J., \& Clemens, M. K. (2006). Health spending projections through 2015: Changes on the horizon. Health Affairs, 25, 61-73.

Bosma, H. L., Marmot, M. G., Hemingway, H., Nicholson, A. C., Brunner, E., \& Stansfeld, S. A. (1997). Low job control and risk of coronary heart disease in Whitehall II (prospective cohort) study. British Medical Journal, 314, 558565.

Bosma, H. L., Stansfeld, S. A., \& Marmot, M. G. (1998). Job control, personal 
characteristics, and heart disease. Journal of Occupational Health

Psychology, 3, 402-409.

Bowen, D. E., Gilliland, S. W., \& Folger, R. (2000). HRM and service fairness: How being fair with employees spills over to customers. Organizational Dynamics, 27(3), 7-23.

Brief, A. P., \& Weiss, H. M. (2002). Organizational behavior: Affect in the workplace. Annual Review of Psychology, 53, 279-307.

Brisson, C., Larocque, B., Moisan, J., Vezina, M., \& Dagenais, G. R. (2000). Psychosocial factors at work, smoking, sedentary behavior, and body mass index: A prevalence study among 6995 white collar workers. Journal of Occupation and Environmental Medicine, 42, 40-46.

Brotheridge, C. M. (2003). The role of fairness in mediating the effects of voice and justification on stress and other outcomes in a climate of organizational change. International Journal of Stress Management, 10, 253-268.

Cahill, J. (1992). Computers and stress reduction in social service workers in New Jersey. In V. Di Martine (Ed.) Preventing stress at work: Conditions of work digest (Vol. 11, pp.197-203). Geneva: International Labour Office.

Colquitt, J. A. (2001). On the dimensionality of organizational justice: A construct validation of a measure. Journal of Applied Psychology, 86, 386-400.

Colquitt, J. A., Conlon, D. E., Wesson, M. J., Porter, C. O. L. H., \& Ng, K. Y. (2001). Justice at the millennium: A meta-analytic review of 25 years of organizational justice research. Journal of Applied Psychology, 86, 425-445.

Colquitt, J. A., \& Greenberg, J. (2003). Organizational justice: A fair assessment of the 
state of the literature. In J. Greenberg (Ed.), Organizational Behavior: The State of the Science (pp. 165-210). Mahwah, NJ: Erlbaum.

Communications Workers of America (2011). Occupational Stress and Workplace. Retrieved March 30, 2013, from, http://www.cwaunion.org/pages/Occupational Stress and the Workplace

Conn, V. S., Hafdahl, A. R., Cooper, P. S., Brown, L. M., \& Lusk, S. L. (2009). Metaanalysis of workplace physical activity interventions. American Journal of Preventative Medicine, 37, 330-339.

Cranny, C. J., Smith, P. C., \& Stone, E. (1992). Job satisfaction: How people feel about their jobs.

Cropanzano, R., Goldman, B. M., \& Benson, L. III. (2005). Organizational justice. In J. Barling, E. K., Kelloway, \& M. R. Frone (Eds.), Handbook of work stress (pp. 6388). Thousand Oaks, CA: Sage Publications.

Cropanzano, R., \& Folger, R. (1991). Procedural justice and worker motivation. In R. M. Steers \& L. W. Porter (Eds.), Motivation and work behavior, 5, 131-143.

Cropanzano, R., \& Greenberg, J. (1997). Progress in organizational justice: Tunneling through the maze. In C. Cooper \& I. Robertson (Eds.), International Review of Industrial and Organizational Psychology (pp. 317-372). New York, NY: Wiley.

DHHS (U.S. Department of Health and Human Services) (1996). Physical activity and health: A report of the Surgeon General. Office of the Surgeon General, Centers for Disease Control and Prevention, Atlanta, GA.

DHHS (U.S. Department of Health and Human Services) (2000). Healthy people 
2010. Office of Disease Prevention and Health Promotion, Washington,

D.C.

DHHS (U.S. Department of Health and Human Services) (2002). Physical activity fundamental to preventing disease. Office of the Assistant Secretary for Planning and Evaluation, Washington, D. C., June.

Daley, A. J., Parfitt, G. (1996). Good health-is it worth it? Mood states, physical wellbeing, job satisfaction and absenteeism in members and non-members of a British corporate health and fitness club. Journal of Occupational and Organizational Psychology, 69, 121-134.

Danna, K., \& Griffin, R. W. (1999). Health and well-being in the workplace: A review and synthesis of the literature. Journal of Management, 25, 357- 384.

DeConinck, J. B., \& Stilwell, C. D. (2004). Incorporating organizational justice, role states, pay satisfaction and supervisor satisfaction in a model of turnover intentions, Journal of Business Research, 57, 225-231.

de Geus, E. J., Van Doornen, L. J., \& Orlebeke, J. F. (1993). Regular exercise and aerobic fitness in relation to psychological make-up and physiological stress reactivity. Psychosomatic Medicine, 55, 347-363.

de Jonge, J., Bosma, H., Peter, R., \& Siegrist, J. (2000). Job strain, effort-reward imbalance and employee well-being: A large-scale cross sectional study. Social Science and Medicine, 50, 1317-1327.

de Jonge, J., \& Kompier, M. A. J. (1997). A critical examination of the demand-control -support model from a work psychological perspective. International Journal of Stress Management, 4, 235-258. 
de Lange, A. H., Taris, T. W., Kompier, M. A. J., Houtman, I. L. D., \& Bongers, P. M. (2003). The very best of the millennium: Longitudinal research and the demand-control-(support) model. Journal of Occupational Health Psychology, 8, 282-305.

Demerouti, E., Bakker, A. B., Nachreiner, F., \& Schaufeli, W. B. (2001). The job demands-resources model of burnout. Journal of Applied Psychology, 86, 499-512.

Deutsch, M. (1975). Equity, equality, and need: What determines which value will be used as the basis for distributive justice? Journal of Social Issues, 31, 137-149.

De Vogli, R., Brunner, E., \& Marmot, M. G. (2007). Unfairness and the social gradient of metabolic syndrome in the Whitehall II study. Journal of Psychosomatic Research, 63, 413-419.

Dunham, R. B., Pierce, J. L., \& Castaneda, M. B. (1987). Alternative work schedules: Two field quasi-experiments. Personnel Psychology, 40, 215-242.

Eek, F., \& Ostergren, P. O. (2009). Factors associated with BMI change over five years in a Swedish adult population. Results from the Scandinavian Public Health Cohort Study. Scandinavian Journal of Public Health; 37, 532-44.

Elliot, D. L., Goldberg, L., Kuehl, K. S., Moe, E. L., Breger, R. K. R., \& Pickering, M. A. (2007). The PHLAME (promoting healthy lifestyles: Alternative models' effects) firefighter study: Outcomes of two models of behavior change. Journal of Occupational Environmental Medicine, 49, 204-213.

Elovainio, M., Kivimaki, M., \& Helkama, K. (2001). Organizational justice evaluations, job control, and occupational strain. Journal of Applied Psychology, 86, 418- 
424.

Elovainio, M., Kivimaki, M., Puttonen, S., Lindholm, H., Pohjonen, T., \& Sinervo, T. (2006). Organizational injustice and impaired cardiovascular regulation among female employees. Occupational and Environmental Medicine, 63, $141-144$.

Elovainio, M., Kivimaki, M., Steen, N., \& Kalliomaki-Levanto, T. (2000).

Organizational and individual factors affecting mental health and job satisfaction: A multilevel analysis of job control and personality. Journal of Occupational Health Psychology, 5, 269-277.

Elovainio, M., Kivimaki, M., Steen, N., \& Vahtera, J. (2004). Job decision latitude, organizational justice and health: Multilevel covariance structure analysis. Social Science \& Medicine, 58, 1659-1669.

Elovainio, M., Kivimaki, M., Vahtera, J., (2002). Organizational justice: evidence of a new psychosocial predictor of health. American Journal of Public Health, 92, 105-108.

Elovainio, M., Kivimaki, M., Vahtera, J., Keltikangas-Jarvinen, L., \& Virtanen, M. (2003). Sleeping problems and health behaviors as mediators between organizational justice and health. Health Psychology, 22, 287-293.

Faragher, E. B., Cass, M., \& Cooper, C. L. (2005). The relationship between job satisfaction and health: A meta-analysis. Occupational Environmental Medicine, 62, 105-112.

Faul, F., Erdfelder, E., Lang, A.G., \& Buchner, A. (2009). G*Power 3: A flexible statistical power analysis program for the social, behavioral, and biomedical 
sciences. Behavior Research Methods, 39, 175-91.

Fisher, V. E., \& Hanna, J. V. (1931). The Dissatisfied Worker. New York: Macmillan.

Folger, R. (1977). Distributive and procedural justice: Combined impact of "voice" and improvement on experienced inequity. Journal of Personality and Social Psychology, 35, 108-119.

Folger, R. \& Cropanzano, R. (1998). Organizational Justice and Human Resource Management. Thousand Oaks, CA: Sage Publications, Inc.

Folger, R., \& Cropanzano, R. (2001). Fairness theory: Justice as accountability. In J. Greenberg \& R. Cropanzano (Eds.), Advances in Organizational Justice (pp. 155). Stanford, CA: Stanford University Press.

Folger, R., \& Konovsky, M. A. (1989). Effects of procedural and distributive justice on reactions to pay raise decisions. Academy of Management Journal, 32, 115130.

Ford, D. K., Truxillo, D. M., \& Bauer, T. N. (2009). Rejected but still there: Shifting the focus in applicant reactions to the promotional context. International Journal of Selection and Assessment, 17, 402-416.

Fox, M. L., Dwyer, D. J., \& Ganster, D. C. (1993). Effects of stressful job demands and control on physiological and attitudinal outcomes in a hospital setting. Academy of Management Journal, 36, 289-318.

Francis, L., \& Barling, J. (2005). Organizational injustice and psychological strain. Canadian Journal of Behavioral Science, 37, 250-261.

Fransson E. I., Keikkilä, K., Nyberg, S. T., et al. (2012). Job strain as a risk factor for leisure-time physical inactivity: An individual participant meta-analysis of up 
to 170,000 men and women. American Journal of Epidemiology, 176, 1078-1089.

Frone, M. R. (2003). Work-Family Balance. In J. C. Quick \& L. E. Tetrick (Eds.), Handbook of Occupational Health Psychology, (pp. 143-162). Baltimore, MD: United Book Press.

Ganster, D. C., Fox, M. L., \& Dwyer, D. J. (2001). Explaining employees' health care costs: a prospective examination of stressful job demands, personal control, and physiological reactivity. Journal of Applied Psychology, 86, 954.

Ganster, D. C., \& Rosen, C. C. (2013). Work stress and employee health: A multidisciplinary review. Journal of Management, 39, 1085-1122.

Garland, E. L., Fredrickson, B., Kring, A. M., Johnson, D. P., Meyer, P. S., \& Penn, D. L. (2010). Upward spirals of positive emotions counter downward spirals of negativity: insights from the broaden-and-build theory and affective neuroscience on the treatment of emotion dysfunctions and deficits of psychopathology. Clinical Psychological Review, 30, 849-864.

Georges, E., Wear, M. L., \& Mueller, W. H. (1992). Body fat distribution and job stress in Mexican-American men of the Hispanic Health and Nutrition Examination Survey. American Journal of Human Biology, 4, 657-667.

Gilliland, S. W. (1993). The perceived fairness of selection systems: An organizational justice perspective. Academy of Management Review, 18, 694734.

Gimeno, D., Tabak, A. G., Ferrie, J. E., Shipley, M. J., De Vogli, R., Elovainio, M., Vahtera, J., Marmot, M. G., \& Kivimaki, M. (2010). Justice at work and metabolic 
syndrome: The Whitehall II study. Occupational Environmental Medicine, $67,256-262$.

Goetzel, R. Z., Guindon, A. M., Turshen, J., \& Ozminkowski, R. J. (2001). Health and productivity management: Establishing key performance measures, benchmarks, and best practices. Journal of Occupational and Environmental Medicine, 43, $10-17$.

Greenberg, J. (1990). Organizational justice: Yesterday, today, and tomorrow. Journal of Management, 16, 399-432.

Greenberg, J. (1993a). The social side of fairness: Interpersonal and informational classes of organizational justice. In: Cropanzano, R. (Ed.), Justice in the Workplace (pp. 79-106). Hillsdale, NJ: Erlbaum.

Greenberg, J. (2004). Stress fairness to fare no stress: Managing workplace stress by promoting organizational justice. Organizational Dynamics, 33, 352-365.

Greenberg, J. (2006). Losing sleep over organizational injustice: Attenuating insomniac reactions to underpayment inequity with supervisory training in interactional justice. Journal of Applied Psychology, 91, 58-69.

Hackman, J. R., \& Oldham, G. R. (1975). Development of the job diagnostic survey. Journal of Applied Psychology, 60, 159-170.

Hagger, M. S., Wood, C. W., Stiff, C., \& Chatzisarantis, N. L. D. (2010). Self-regulation and self-control in exercise: The strength-energy model. International Review of Sport and Exercise Psychology, 3, 62-86.

Hammer, L. B., Cullen, J. C., Neal, M. B., Sinclair, R. R. \& Shafiro, M. V. (2005a). The longitudinal effects of work-family conflict and positive spillover on 
depressive symptoms among dual-earner couples. Journal of Occupational Health Psychology, 10, 138-154.

Hammer, L. B., \& Sauter, S. (2013). Total worker health and work-life stress. Journal of Occupational and Environmental Medicine, 55, S25-S29.

Harris, L. (2000). Procedural justice and perceptions of fairness in selection practice. International journal of selection and assessment, 8, 148-158.

Harrison, D. A., Newman, D. A., \& Roth, P. L. (2006). How important are job attitudes? Meta-analytic comparisons of integrative behavioral outcomes and time sequences. Academy of Management Journal, 49, 305-325.

Heaney, C. (2003). Worksite health interventions: Targets for change and strategies for attaining them. In J. C. Quick \& L. E. Tetrick (Eds.), Handbook of Occupational Health Psychology (pp. 265-287). Baltimore, MD: United Book Press.

Hellerstedt, W. L. \& Jeffery, R. W. (1997). The association of job strain and health behaviours in men and women. International Journal of Epidemiology, 26, $575-583$.

Hemingway, H., \& Marmot, M. (1999). Psychosocial factors in the aetiology and prognosis of coronary heart disease: A systematic review of prospective cohort studies. British Medical Journal, 318, 1460-1467.

Hendrix, W. H., Robbins, T., Miller, J., \& Summers, T. P. (1998). Effects of procedural and distributive justice on factors predictive of turnover. Journal of Social Behavior \& Personality, 13, 611-632.

Hersey, R. B. (1932). Workers' Emotions in Shop and Home: A Study of Individual 
Workers from the Psychological and Physiological Standpoint.

Philadelphia: Univ. Penn. Press.

Hoel, H., Rayner, C., \& Cooper, C. L., (1999). Workplace bullying. In C. L. Cooper \& I. T. Robertson (Eds.), International review of industrial and organizational psychology (Vol. 14). Chichester, UK: Wiley.

House, J. S., (1981). Work stress and social support. Reading, MA: Addison-Wesley. Hoppock, R. (1935). Job Satisfaction. New York: Harper.

Horowitz, M. J., Benfari, R., Hulley, S., Blair, S., Alvarez, W., Borhani, N., Reynolds, A., \& Simon, N. (1979). Life events, risk factors, and coronary disease. Psychosomatics, 20, 586-592.

Howard. L. W., \& Cordes, C. L. (2010). Flight from unfairness: Effects of perceived injustice on emotional exhaustion and employee withdrawal. Journal of Business Psychology, 25, 409-428.

Hulin, C. L. \& Judge, T. A. (2003). Job Attitudes. W. C. Borman, D. R. Ilgen, \& R. J. Klimoski (Eds.). In Handbook of Psychology: Vol. 12. Industrial and Organizational Psychology (pp. 255-276). Hoboken: NJ: John Wiley \& Sons, Inc.

Humphrey, S. E., Nahrgang, J. D., \& Morgeson, F. P. (2007). Integrating motivational, social, and contextual work design features: a meta-analytic summary and theoretical extension of the work design literature. Journal of Applied Psychology, 92, 1332. 
Hurrell, J. J. (2005). Organizational stress intervention. In J. Barling, E. K. Kelloway, \& M. R. Frone (Eds.), Handbook of work stress (pp. 623-640). Thousand Oaks, CA: Sage Publications.

Ishizaki, M., Nakagawa, H., Morikawa, Y., Honda, R., Yamada, Y., \& Kawakami, N. (2008). Influence of job strain on changes in body mass index and waist circumference - 6-year longitudinal study. Scandinavian Journal of Work Environment and Health, 34, 288-296.

Jex, S. M., \& Britt, T. W. (2008). Chapter 10: Leadership and influence processes. Organizational Psychology: Scientist-Practitioner Approach. New Jersey, John Wiley \& Sons Inc., 303-338.

Jex, S. M., \& Crossley, C. D. (2005). Organizational consequences. In J. Baring, E. K. Kelloway, \& M. Frone (Eds.), Handbook of work stress (pp.575-599). Thousand Oaks, CA: Sage Publications.

Jones, D. A., \& Martens, M. (2007). The mediating role of overall fairness and the moderating role of trust certainty in justice-criteria relationships: Testing fundamental tenets of fairness heuristic theory. Best Paper Proceedings of the 67th Annual Meeting of the Academy of Management. Philadelphia, PA.

Jonsson, D., Rosengren, A., Dotevall, A., Lappas, G., \& Wilhelmsen, L. (1999). Job control, job demands and social support at work in relation to cardiovascular risk factors in MONICA 1995, Goteborg. Journal of Cardiovascular Risk, 6, 379-385.

Johnson, W., Kyvik, K. O., Skytthe, A., Deary, I. J., Sorensen, T. I. A. (2011). Education modifies genetic and environmental influences on BMI. PLOS ONE, 6(1): 
e16290.

Judge, T. A., \& Bono, J. E. (2001). Relationship of core self-evaluations traits—selfesteem, generalized self-efficacy, locus of control, and emotional stabilitywith job satisfaction and job performance: A meta-analysis. Journal of Applied Psychology, 86(1), 80.

Judge, T. A., \& Colquitt, J. A. (2004). Organizational justice and stress: The mediating role of work-family conflict. Journal of Applied Psychology, 89, 395-404.

Judge, T. A., Parker, S. K., Colbert, A. E., Heller, D., \& Ilies, R. (2002). Job satisfaction: A cross-cultural review. Anderson, N., Ones, DS, Sinangil, HK, Viswesvaran, C. Handbook of industrial, work, and organizational psychology, 25-52.

Judge, T. A., Thoresen, C. J., Bono, J. E., \& Patton, G. K. (2001). The job satisfaction-job performance relationship: A qualitative and quantitative review. Psychological Bulletin, 127, 376-407.

Juster, R. P., McEwen, B. S., \& Lupien, S. J. (2010). Allostatic load biomarkers of chronic stress and impact on health and cognition. Neuroscience and Biobehavioral Reviews, 35, 2-16.

Karasek, R. A. (1979). Job demands, job decision latitude, and mental strain: Implications for job redesign. Administrative Science Quarterly, 24, 285-306.

Karasek, R. A. (1985). Job Content Instrument: Questionnaire and User's Guide. Los Angeles; University of South Carolina.

Karasek, R. A. (1990). Lower health risk with increased job control among white collar workers. Journal of organizational behavior, 11, 171-185.

Karasek, R. A. (1998). The Job Content Questionnaire (JCQ): An instrument for 
internationally comparative assessments of psychosocial job characteristics. Journal of Occupational Health Psychology, 3, 322-355.

Karasek, R. A., Brisson, C., Kawakami, N., Houtman, I., Bongers, P., \& Amick, B. (1998). The Job Content Questionnaire (JCQ): An instrument for Internationally comparative assessments of psychosocial job characteristics. Journal of Occupational Health Psychology, 3, 322-355.

Karasek, R. A., Schwartz, J., \& Pieper, C. (1983). Validation of a survey instrument for job-related cardiovascular illness. New York: Columbia University, Department of Industrial Engineering and Operations Research.

Karasek, R. A., \& Theorell, T. (1990). Health work: Stress, productivity, and the reconstruction of working life. New York, NY: Basic Books.

Kasl, S.V. (1987). Methodologies in stress and health: Past difficulties, present dilemmas, future directions. In S. Kasl \& C. Cooper (Eds.), Stress and Health: Issues in Research Methodology. Chichester, England: John Wiley \& Sons, Inc.

Kenny, D. T., Carlson, J. G., McGuigan, F. J., \& Sheppard, J. L. (eds.). (2000). Stress and health: Research and clinical applications. Amsterdam: Harwood Academic Publishers.

Kelloway, E. K., Sivanathan, N., Francis, L., \& Barling, J. (2005). Poor leadership. In J. Barling, E. K. Kelloway, \& M. R. Frone (Eds.), Handbook of work stress (pp. 89112). Thousand Oaks, CA: Sage Publications.

Kim, T., \& Leung, K. (2007). Forming and reacting to overall fairness: A crosscultural comparison. Organizational Behavior and Human Decision Processes, 104, 83-95. 
Kirdcaldy, B. D., Cooper, C. L., Shephard, R. J., Brown, J. S. (1994). Exercise, job satisfaction and well-being among superintendent police officers. European Review of Applied Psychology, 44, 117-123.

Kivimaki, M., Elovainio, M., Vahtera, J., Virtanen, M., \& Stansfeld, S. A. (2003a). Association between organizational inequity and incidence of psychiatric disorders in female employees. Psychological Medicine, 33, 319-326.

Kivimaki, M., Elovainio, M., Vahtera, J., \& Ferrie, J. E. (2003). Organizational justice and health of employees: Prospective cohort study. Occupational and Environmental Medicine, 60, 27-33.

Kivimaki, M., Ferrie, J. E., Brunner, E., Head, J., Shipley, M. J., Vahtera, J., \& Marmot, M. G. (2005). Justice at work and reduced risk of coronary heart disease among employees: The Whitehall II study. Archives of Internal Medicine, 165, 22452251.

Kivimaki, M., Ferrie, J. E., Shipley, M., Gimeno, D., Elovainio, M., de Vogli, R., Head, J. (2008). Effects on blood pressure do not explain the association between organizational justice and coronary heart disease in the Whitehall II Study. Psychosomatic Medicine, 70, 1-6.

Kivimaki, M., Head, J., Ferrie, J. E., Shipley, M., Brunner, E., Vahtera, J., \& Marmot, M. G. (2006). Work stress, weight gain and weight loss: Evidence for bidirectional effects of job strain on body mass index in the Whitehall II study. International Journal of Obesity, 30, 982-987.

Kivimaki, M., Leino-Arjas, P., Luukkonen, R., Riihimaki, H., Vahtera, J., \& Kirjonen, J. 
(2002). Work stress and risk of cardiovascular mortality: Prospective cohort study of industrial employees. British Medical Journal, 325, 857-860.

Kivimaki, M., Vahtera, J., Thomson, L., Griffiths, A., Cox, T., \& Pentti, J. (1997). Psychosocial factors predicting employee sickness absence during economic decline. Journal of Applied Psychology, 82, 858-872.

Korkeila, M., Kaprio, J., Rissanen, A., Koskenvuo, M., \& Sorenson, T. (1998). Predictors of major weight gain in adults Finns: stress, life satisfaction and personality traits. International Journal of Obesity, 22, 949-957.

Kornhauser, A. W., \& Sharp, A. A. (1932). Employee attitudes: suggestions from a study in a factory. Personnel Journal, 10, 393-404.

Kouvonen, A., Kivimaki, M., Cox, S. J., Cox, T., \& Vahtera, J. (2005). Relationship between work stress and body mass index among 45,810 female and male employees. Psychosomatic Medicine, 67, 577-83.

Kouvonen, A., Kivimaki, M., Elovainio, M., Virtanen, M., Linna, A., \& Vahtera, J. (2005). Job strain and leisure-time physical activity in female and male public sector employees. Preventative Medicine, 41, 532-539.

Kristenson, M., Orth-Gomer, K., Kucienskiene, Z., Bergdahl, B., Calcauskas, H., Balnyiene, I., \& Olsson, A. G. (1998). Attenuated cortisol response to a standardized stress test in Lithuanian vs Swedish men: the LiVicordia study. International Journal of Behavioral Medicine, 5, 17-30.

Kristof-Brown, A. L., Zimmerman, R. D., \& Johnson, E. C. (2005). Consequences of individuals' fit at work: A meta-analysis of person-job, person-organization, person-group, and person-supervisor fit. Personnel Psychology, 58(2), 281- 
342.

Kujala, U. M., Kaprio, J., Sarna, S., \& Koskenvuo, M. (1998). Relationship of leisuretime physical activity and mortality. Journal of America Medical Association, $279,440-444$.

Kuper, H., \& Marmot, M. (2003). Job strain, job demands, decision latitude, and risk of coronary heart disease with the Whitehall II study. Journal of Epidemiology and Community Health, 57, 147-153.

Laitinen, J., Ek, E., Sovio, U. (2002). Stress-related eating and drinking behavior and body mass index and predictors of this behavior. Preventative Medicine, 34, $29-39$.

Lallukka, T., Laaksonen, M., Martikainen, P., Sarlio-Lahteenkorva, S., \& Lahelma, E. (2005). Psychosocial working conditions and weight gain among employees. International Journal of Obesity Related to Metabolic Disorders, 29, 909-915.

Landers, D.M., \& Arent, S.M. (2007). Physical activity and mental health. In G.

Tenenbaum \& C. Ecklund (Eds.), The Handbook of Sport Psychology (pp. 469491). Hoboken, NJ: John Wiley and Sons.

Landsbergis, P. A., Schnall, P. L., Deitz, D. K., Warren, K., Pickering, T. G., \& Schwartz, J. E. (1998). Job strain and health behaviors: results of a prospective study. American Journal of Health Promotion, 12, 237-245.

Langlie, J. K. (1977). "Social networks, health beliefs, and preventive health behavior." Journal of Health and Social Behavior, 244-260.

Lepore, S. J. (1995). Cynicism, social support, and cardiovascular reactivity. Health Psychology, 14, 10-16. 
Leung, K., Smith, P. B., Wang, Z., \& Sun, H. (1996). Job satisfaction in joint venture hotels in China: An organizational justice analysis. Journal of International Business Studies, 27(5), 947-962.

Leventhal, G. S. (1976). Fairness in social relationships. In J. W. Thibault, J. T. Spence, \& R. C. Carton (Eds.), Contemporary topics in social psychology (pp. 211-239). Morristown, NJ: General Learning Press.

Leventhal, G. S. (1980). What should be done with equity theory? In K. J. Gergen, M.S. Greenberg, \& R. H. Willis (Eds.), Social exchange: Advances in theory and research (pp. 27-55). New York: Plenum.

Leventhal, G. S., Karuza, J., \& Fry, W. R. (1980). Beyond fairness: A theory of allocation preferences. In G. Mikula (Ed.), Justice and social interaction (pp. 167-218). New York: Springer-Verlag.

Lind, E.A. (2001). Fairness heuristic theory: Justice judgments as pivotal cognitions in organizational settings. In: J. Greenberg \& R. Cropanzano (Eds.), Advances in Organizational Justice (pp. 56-88). Stanford, CA: Stanford University Press.

Lind, E. A., \& Tyler, T. (1988). The social psychology of procedural justice. New York: Plenum.

Locke, E. A. (1976). The nature and causes of job satisfaction. In M. D. Dunnette (Ed.), In Handbook of Industrial/ Organizational Psychology (pp. 1297-1349). Chicago: Rand-McNally. 1st edition.

Luger, A., Deuster, P. A., Kyle, S. B., Gallucci, W. T., Montgomery, L. C., Gold, P. W., ... \& Chrousos, G. P. (1987). Acute hypothalamic-pituitary-adrenal responses to the stress of treadmill exercise. New England Journal of Medicine, 316(21), 
1309-1315.

Lundberg, U. (2005). Stress hormones in health and illness: The roles of work and gender. Psychoneuroendocrinology, 30, 1017-1021.

Lundberg, U., \& Johansson, G. (2000). Stress and health risks in repetitive work and supervisory monitoring work. In Backs, R., Boucsein, W. (Eds.), Engineering Psychophysiology: Issues and Applications. Lawrence Erlbaum Associates, New Jersey, pp339-359.

Lupien, S. J. Ouellet-Morrin, I., Hupbach, A. Tu, M. T., Buss, C., Walker, D., Pruessner, J., \& McEwen, B. S. (2006). Beyond the stress concept: Allostatic load - a developmental biological and cognitive perspective. In D. Cicchetti \& D. J. Cohen (Eds.), Developmental Psychopathology, volume 2: Developmental Neuroscience: 578-628. Hoboken, NJ: John Wiley.

Markovsky, B. (1988). Anchoring justice. Social Psychology Quarterly, 51, 213-224.

Marmot, M. G., \& Brunner, E. (2005). Cohort profile: The Whitehall II study. International Journal of Epidemiology, 34, 251-256.

Marmot, M. G., Smith, D. G., Stansfeld, S., Patel, C., North, F., Head, J., White, I., Brunner, E., \& Feeney, A. (1991). Health inequalities among British civil servants: The Whitehall II study. Lancet, 337, 1387-1393.

Masterson, S. S. (2001). A trickle-down model of organizational justice: relating employees' and customers' perceptions of and reactions to fairness. Journal of Applied Psychology, 86(4), 594.

Matarazzo, J. D. (1982). Behavioral health's challenge to academic, scientific, and professional psychology. American Psychologist, 37, 1-14. 
McColl-Kennedy, J. R., \& Sparks, B. A. (2003). Application of fairness theory to service failures and service recovery. Journal of Service Research, 5, 251-266.

McEwen, B. S., \& Stellar, E. (1993). Stress and the individual: Mechanisms leading to disease. Archives of Internal Medicine, 153: 2093-2101.

McFarlin, D. B., \& Rice, R. W. (1992). The role of facet importance as a moderator in job satisfaction processes. Journal of Organizational Behavior, 13, 41-54.

McFarlin, D. B., \& Sweeney, P. D. (1992). Distributive and procedural justice as predictors of satisfaction with personal and organizational outcomes. Academy of Management Journal, 35, 626-637.

McLeroy, K. R., Gottlieb, N. H. \& Heaney, C. A. (2002). Social health in the workplace. In M. P. O'Donnell (Ed.), Health promotion in the workplace (3rd ed., pp. 459492). Albany, NY: Delmar.

Meijman, T. F., Mulder, G., \& Van Dormolen, M. (1992). Workload of driving examiners: A psychophysiological field study. In H. Kragt (Ed.), Enhancing industrial performances (pp. 245-260). London: Taylor \& Francis.

Moorman, R. H. (1991). Relationship between organizational justice and organizational citizenship behavior: Do fairness perceptions influence employee citizenship? Journal of Applied Psychology, 76, 845-855.

Moyle, P. J. (1998). Longitudinal influences of managerial support on employee wellbeing. Work \& Stress, 12, 29-49.

Muller, D. Judd, C. M., \& Yzerbyt, V. Y. (2005). When moderation is mediated and mediation is moderated. Journal of Personality and Social Psychology, 89, 852863. 
Muraven, M., \& Baumeister, R. F. (2000). Self-regulation and depletion of limited resources: Does self-control resemble a muscle? Psychological Bulletin, 126(2), 247.

National Center for Chronic Disease Prevention and Health Promotion, Division for Heart Disease and Stroke Prevention. (2013, June). Learn about heart disease. Retrieved April 10, 2013, from http://www.cdc.gov/heartdisease/

National Center for Chronic Disease Prevention and Health Promotion, Division of Nutrition, Physical Activity, and Obesity. (2013, August). Overweight and Obesity for Professionals: Data and Statistics. Retrieved April 10, 2013, from http://www.cdc.gov/obesity/data/adult.html

National Center for Disease Control and Prevention (2009). Health, United States, 2009: With special feature on medical technology. Retrieved May 8, 2013, from http://www.cdc.gov/nchs/data/hus/hus09.pdf

National Heart, Lung, and Blood Institute (2012, August). Who is at risk for high blood pressure? Retrieved May 15, 2013, from National Institute of Health: http://www.nhlbi.nih.gov/health/health-topics/topics/hbp/atrisk.html

Naumann, S. E., \& Bennett, N. (2000). A case for procedural justice climate: Development and test of a multilevel model. Academy of Management Journal, 43, 881-889.

Nelson, D. L., \& Simmons, B. L. (2003). Health psychology and work stress: A more positive approach. In J. C. Quick \& L. E. Tetrick (Eds.), Handbook of Occupational Health Psychology (pp. 97-119). Baltimore, MD: United Book Press. 
Netterstrom, B., Kristensen, T. S., Damsgaard, M. T., Olsen, O., \& Sjol, A. (1991). Job

strain and cardiovascular risk factors: a cross sectional study of employed Danish men and women. British Journal of Industrial Medicine, 48, 684-689.

Niedhammer, I., Goldberg, M., Leclerc, A., David, S., Bugel, I., Landre, M. F. (1998). Psychosocial work environment and cardiovascular risk factors in an occupational cohort in France. Journal of Epidemiology and Community Health, 52, 93-100.

Nyberg, S. T., Heikkila, K., \& Fransson, E. I., et al., (2012). Job strain in relation to body mass index: pooled analysis of 160000 adults from 13 cohort studies. Journal of Internal Medicine, 272, 65-73.

Nyberg, S. T., Fransson, E. I., Heikkila, K., Alfredsson, L., Casini, A., Clays, E., et al. (2013). Job strain and cardiovascular disease risk factors: Meta-analysis of Individual- participant data from 47,000 men and women. PLOS ONE, 8, e67323.

Oaten, M., \& Cheng, K. (2006). Longitudinal gains in self-regulation from regular physical exercise. British Journal of Health Psychology, 11(4), 717-733.

Offermann, L. R., \& Hellmann, P. S. (1996). Leadership behavior and subordinate stress: A 360 view. Journal of Occupational Health Psychology, 1, 382-390.

Ossip-Klein, D. J., Doyne, E. J., Bowman, E. D., Osborn, K. M., \& Neimeyer, R. A. (1989). Effects of running or weight lifting on self-concept in clinically depressed women. Journal of Consulting and Clinical Psychology, 57(1), 158.

Overgaard, D., Gyntelberg, F., \& Heitmann, B. L. (2004). Psychological workload and 
body weight: is there an association? A review of the literature. Occupational Medicine, 54, 35-41.

Parkes, K. R., Menham, C. A., \& Rabenau, C. V. (1994). Social support and the demand-discretion model of job stress: Tests of additive and interactive effects in two samples. Journal of Vocational Behavior, 44, 91-113.

Parkes, K. R., \& Sparkes, T. I. (1998). Organizational interventions to reduce work stress: Are they effective? (HSE Contract Research Report 193). Colegate, UK: Her Majesty's Stationery Office.

Payne, N., Jones, F., Harris, P. R. (2005). The impact of job strain on the predictive validity of the theory of planned behavior: An investigation of exercise and healthy eating. British Journal of Health Psychology, 10, 115-131.

Pillai, R., Schriesheim, C. A., \& Williams, E. S. (1999). Fairness perceptions and trust as mediators for transformational and transactional leadership: A twosample study. Journal of Management, 25, 897-934.

Podsakoff, P. M., MacKenzie, S. B., Jeong-Yeon, L., \& Podsakoff, N. P. (2003). Common method biases in behavioral research: A critical review of the literature and recommended remedies. Journal of Applied Psychology, 88, 879-903.

Pratt, L. (1971). The relationship of socioeconomic status to health. American Journal of Public Health, 61, 281-291.

Price, J. L., \& Mueller, C. W. (1986). Handbook of Organizational Measurement. Marshfield, MA: Pittman.

Quick, J. C., Quick, J. D., Nelson, D. L., \& Hurrell, J. J. (1997). Stress in organizations. In J. C. Quick, J. D. Quick, D. L. Nelson \& J. J. Hurrell (Eds.), Preventative Stress 
Management in Organizations (pp. 1-20). Washington, D. C.: American Psychological Association.

Reed, D. M., LaCroix, A. Z., Karasek, R. A., Miller, D., \& MacLean, C. A. (1989). Occupational strain and the incidence of coronary heart disease. American Journal of Epidemiology, 129, 495-502.

Rethorst, C. D., Wipfli, B. M., \& Landers, D. M. (2009). The antidepressive effects of exercise. Sports Medicine, 39(6), 491-511.

Richman, J. A., Flaherty, J. A., Rospenda, K. M., \& Christensen, M. (1992). Mental health consequences and correlates of medical student abuse. Journal of the American Medical Association, 267, 692-694.

Robbins, J. M., Ford, M. T., \& Tetrick, L. E. (2012). Perceived unfairness and employee health: A meta-analytic integration. Journal of Applied Psychology, 97, 235272.

Robbins, T. W., \& Fray, P. J. (1980). Stress-induced eating: fact, fiction or misunderstanding? Appetite, 1, 103-133.

Robertson, Q. M., Moye, N. A., \& Locke, E. A. (1999). Identifying a missing link between participation and satisfaction: The mediating role of procedural justice perceptions. Journal of Applied Psychology, 84,585-593.

Roethlisberger, F. J., \& Dickson, W. J. (1939). Management and the Worker. Cambridge, MA: Harvard Univ. Press.

Rospenda, K. M. (2002). Workplace harassment, services utilization, and drinking outcomes. Journal of Occupational Health Psychology, 7, 141-155.

Sapolsky, R. M. (1998). Why zebras don't get ulcers: An updated guide to stress, stress- 
related disease, and coping. New York: Freeman.

Sauter, S. L., Hurrell, J. J., \& Cooper, C. L. (Eds.). (1989). Job control and worker health. Wiley.

Schachter, S., Silverstein, B., Kozlowski, L. T., Herman, L. P., \& Liebling, B. (1977). Effects of stress on cigarette smoking and urinary pH. Journal of Experimental Psychology: General, 106, 24-30.

Schill, A., \& Chosewood, L. C. (2013). The NIOSH total worker health program: An overview. Journal of Occupational and Environmental Medicine, 55, 8-11.

Schmier, J. K., Jones, M. L., \& Halpern, M. T. (2006). Cost of obesity in the workplace. Scandinavian Journal of Work, Environment \& Health, 32(1), 5-11.

Schnall, P. L., Landsbergis, P. A., \& Baker, D. (1994). Job strain and cardiovascular disease. Annual Review of Public Health, 15, 381-411.

Schweiger, D. M., \& DeNisi, A. S. (1991). Communication with employees following a merger: A longitudinal field experiment. Academy of Management Journal, 34, 110-135.

Searle, B. J., Bright, J. E. H., \& Bochner, S. (1999). Testing the 3-factor mode of occupational stress: The impact of demands, control and social support on a mail sorting task. Work and Stress, 13, 268-279.

Selye, H. (1950). The physiology and pathology of exposure to stress.

Seligman, M. E. P., \& Csikszentmihalyi, M. (2000). Positive psychology. American Psychologist, 55, 5-14. 
Semmer, N. K. (2003). Job stress interventions and organization of work. In J. C. Quick \& L. E. Tetrick (Eds.) Handbook of Occupational Health Psychology (pp. 325-353). Baltimore, MD: United Book Press.

Shao, R., Rupp, D. E., Skarlicki, D. P., \& Jones, K. S. (2013). Managing justice across cultures: A meta-analytic review, a management framework, and a crosscultural research agenda. Journal of Management, 39, 263-301.

Siegrist, J. (1996). Adverse health effects of high-effort/low-reward conditions. Journal of Occupational Health Psychology, 1, 27-41.

Simons, T. \& Roberson, Q. (2003). Why managers should care about fairness: The effects of aggregate justice perceptions on organizational outcomes. Journal of Applied Psychology, 88, 432-443.

Smith, C., Cowan, C., Heffler, S., \& Catlin, A. (2006). National health spending in 2004: Recent slowdown led by prescription drug spending. Health Affairs, 25, 186196.

Smith, P. C., Kendall, L. M., \& Hulin, C. L. (1969). The measurement of satisfaction in work and retirement: A strategy for the study of attitudes.

Sonnentag, S. \& Frese, M. (2003). Stress in organizations. In W. C. Borman, D. R. Ilgen, \& R. J. Klimoski (Eds.), Handbook of Psychology: Vol. 12. Industrial and Organizational Psychology (pp. 453-491). Hoboken: NJ, John Wiley \& Sons, Inc.

Sonnentag, S., \& Jelden, S. (2009). Job stressors and the pursuit of sport activities: a day-level perspective. Journal of Occupational Health Psychology, 14, 165181. 
Spector, P. E., Chen, P. Y., \& O'Connell, B. J. (2000). A longitudinal study of relations

between job stressors and job strains while controlling for prior negative affectivity and strains. Journal of Applied Psychology, 85, 211-218.

Stansfeld, S.A., Bosma, H., Hemingway, H., \& Marmot, M.G. (1998a). Psychosocial work characteristics and social support as predictors of SF-36 health functioning: the Whitehall II Study. Psychosomatic Medicine, 60, 247-255.

Sweeney, D. P., \& McFarlin, D. B. (1993). Workers' evaluation of the "ends" and "means": An examination of four models of distributive justice and procedural justice. Organizational Behavior and Human Decision Processes, $55,23-40$.

Tait, M., Padgett, M. Y., \& Baldwin, T. T. (1989). Job and life satisfaction: A reevaluation of the strength of the relationship and gender effects as a function of the date of the study. Journal of Applied Psychology, 74(3), 502.

Taris, T. W. (2006). Bricks without clay: On urban myths in occupational health psychology. Work \& Stress, 20, 99-104.

Taris, T.W., Kalimo, R. \& Schaufeli, W.B. (2002). Inequity at work: Its measurement and association with worker health. Work and Stress, 16, 287-301.

Taylor, A. (2000). Physical activity, anxiety, and stress. In: Biddle, S.J.H., Fox, K.R., Boutcher, S.H. (Eds.), Physical Activity and Psychological Well-Being. Routledge, London, UK, pp. 10-46.

Tepper, B. J. (2000). Consequences of abusive supervision. Academy of Management Journal, 43, 178-190. 
Tepper, B. J. (2001). Health consequences of organizational injustice: Tests of main

and interactive effects. Organizational Behavior and Human Decision Processes, 86, 197-215.

Theorell, T. (2003). To be able to exert control over one's situation: A necessary condition for coping with stressors. In J. C. Quick \& L. E. Tetrick (Eds.), Handbook of Occupational Health Psychology (pp. 201-220). Baltimore, MD: United Book Press.

Theorell, T., \& Karasek, R. A. (1996). Current issues relating to psychological job strain and cardiovascular disease research. Journal of Occupational Health Psychology, 1, 9-26.

Theorell, T., Tsutsumi, A., Hallquist, J., Reuterwall, C., Hogstedt, C., Fredlund, P., ... \& Johnson, J. V. (1998). Decision latitude, job strain, and myocardial infarction: a study of working men in Stockholm. The SHEEP Study Group. Stockholm Heart epidemiology Program. American Journal of Public Health, 88(3), 382388.

Thibaut, J. W., \& Kelley, H. H. (1959). The social psychology of groups. New York: Wiley.

Thibaut, J. \& Walker, L. (1975). Procedural justice: A psychological analysis. Hillsdale, NJ: Erlbaum.

Thomas, L. T., \& Ganster, D. C. (1995). Impact of family-supportive work variables on work-family conflict and strain: A control perspective. Journal of Applied Psychology, 80, 6-15. 
Torres, S. J., Caryl, M. \& Nowson, A. (2007). Relationship between stress, eating behavior, and obesity. Nutrition, 23, 887-894.

Totterdell, P., Spelten, E., Smith, L., Barton, J., \& Folkard, S. (1995). Recovery from work shifts: How long does it take? Journal of Applied Psychology, 80, 43-57.

Tsutsumi A, Kayaba K, Yoshimura M, Sawada M, Ishikawa, S, Sakai K, et al. (2003). Association between job characteristics and health behaviors in Japanese rural workers. International Journal of Behavioral Medicine, 10, 25-42.

Tyler, J. M., \& Burns, K. C. (2008). After depletion: The replenishment of the self's regulatory resources. Self and Identity, 7(3), 305-321.

Tyler, T. R. (1990). Why people follow the law: Procedural justice, legitimacy, and compliance. New Haven, CT: Yale University Press.

Tyler, T. R. (1994). Psychological models of the justice motive: Antecedents of distributive and procedural justice. Journal of Personality and Social Psychology, 67, 850-863.

Tyler, T. R., \& Blader, S. L. (2003). The group engagement model: Procedural justice, social identity, and cooperative behavior. Personality and Social Psychology Review, 7, 349-361.

Tyler, T. R., \& Degoey, P. (1995). Collective restrain in social dilemmas: Procedural Justice and social identification effects of support for authorities. Journal of Personality and Social Psychology, 69, 482-497.

Tyler, T. R., Degoey, P., \& Smith, H. (1996). Understanding why the justice of group procedures matters: A test of the psychological dynamics of the groupvalue model. Journal of Personality and Social Psychology, 70, 913-930. 
Tyler, T. R. \& Lind, E. A. (1992). A relational model of authority in groups. In M. P.

Zanna (Ed.), Advances in experimental social psychology (25, pp. 115-191). San Diego, CA: Academic Press.

van der Doef, M., \& Maes, S. (1999). The job demand-control (-support) model and psychological well-being: A review of 20 years of empirical research. Work and Stress, 13, 87-114.

Verquer, M. L., Beehr, T. A., \& Wagner, S. H. (2003). A meta-analysis of relations between person-organization fit and work attitudes. Journal of Vocational Behavior, 63(3), 473-489.

Viswesvaran, C., Sanchez, J. I., \& Fisher, J. (1999). The role of social support in the process of stress: A meta-analysis. Journal of Vocational Behavior, 54, 314334.

Wager, N., Fieldman, G., \& Hussey, T. (2003). The effect on ambulatory blood pressure of working under favourably and unfavorably perceived supervisors. Occupational and Environmental Medicine, 60, 468-474.

Wallace, J. C., Edwards, B. D., Shull, A., \& Finch, D. M. (2009). Examining the consequences in the tendency to suppress and reappraise emotions on taskrelated job performance. Human Performance, 22(1), 23-43.

Wamala, S. P., Wolk, A., \& Orth-Gomer, K. (1997). Determinants of obesity in relation to socioeconomic status among middle-aged Swedish women. Preventative Medicine, 26, 734-744.

Wanous, J. P., Reichers, A. E., \& Hudy, M. J. (1997). Overall job satisfaction: how good 
are single-item measures? Journal of Applied Psychology, 82(2), 247.

Wardle, J., Chida, Y., Gibson, E. L., Whitaker, K. L., \& Steptoe, A. (2011). Stress and adiposity: a meta-analysis of longitudinal studies. Obesity, 19, 771-778.

Weiss, H. M., \& Brief, A. P. (2001). Affect at work: A historical perspective. Emotions at work: theory, research and applications for management, 133-171.

Weiss, H. M., \& Cropanzano, R. (1996). Affective events theory: A theoretical discussion of the structure, causes and consequences of affective experiences at work. Research in Organizational Behavior, 18, 1-74.

Williams, A. F., \& Wechsler, H. (1972). "Interrelationship of preventive actions in health and other areas." Health Services Reports, 87, 10, 969-976.

Wipfli, B., \& Ramirez, E. (2013). Stress Reactivity in Humans and Animals: Two Meta-Analyses. International Journal of Exercise Science, 6(2), 7.

Wolpin, J., Burker, R. J., \& Greenglass, E. R. (1991). Is job satisfaction an antecedent or a consequence of psychological burnout? Human Relations, 44, 193-209. World Health Organization (WHO). (April, 1948). Preamble to the Constitution of the World Health Organization. New York, NY: Author. 


\section{Appendix A: SHIP Flyer}

\section{Safety \& Health Improvement Program}

for Crew Leadership, Teams and Individuals

The Portland Water Bureau and the Portland Bureau of TransportationMaintenance Operations have been chosen as partners with Portland State University (PSU) and the Oregon Healthy Workforce Center for a 3-year program. Your organization is participating to contribute to the understanding of ways of improving health, safety and effectiveness on and off the job.

The PSU team of the Oregon Healthy Workforce Center is investigating:

- Common sources of stress at work

- How work impacts personal life and health

- Specific work conditions that help to be more effective both at work and at home

- How to improve workplace safety

\section{How do I participate?}

Program staff from PSU will facilitate program activities. We will coordinate with your supervisor to schedule a convenient time for your participation.

\section{What is involved?}

Safety and Health Improvement Program (SHIP) Individual Process:

- Complete a questionnaire at work during paid work hours (about 40 minutes)

- Participate in a health screening at work during paid work hours (about a half hour), which includes:

- blood pressure, height and weight measures

- As a thank you for your participation, we will give you a $\mathbf{\$ 2 5}$ gift card for completing the survey and health screening.

SHIP Crew Leadership Process:

- Complete a computer-based leadership training

- Track supportive behaviors for two weeks using an iPod 
SHIP Team Process:

- Attend a team process session with your crew

What are the benefits to me?

Our aim is to understand how work affects health and wellness and how work environments might be improved. We expect that this program will result in improved morale, less turnover, enhanced work processes, better health and commitment to safety.

\section{Do I have to participate?}

Participation in the survey and health assessment are voluntary and will not affect your employment in any way. Your participation in SHIP crew leadership and the team process are expected by your organization.

\section{Will the Water Bureau/PBoT see my results?}

No. Your results are completely confidential. Your organization will receive information about the overall study results, but no one individual will be identified. 


\section{Appendix B: Informed Consent Form}

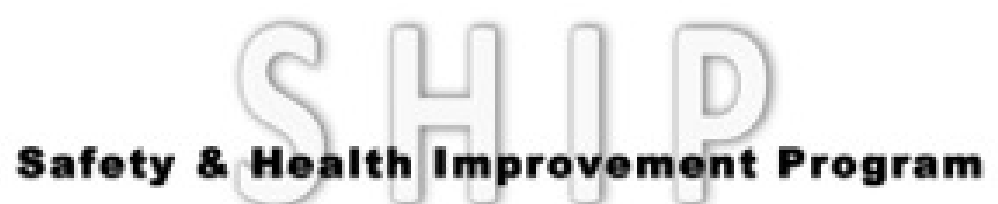

You are invited to participate in a study conducted by Drs. Leslie Hammer, Donald Truxillo, and Todd Bodner at Portland State University. They are studying the factors affecting employees' safety, health, and work experiences. All workers at the Water Bureau and Bureau of Transportation in construction-related fields are invited to participate in the study.

If you decide to participate, we ask that you complete two questionnaires, which should each take about 20 minutes of your time. The surveys contain questions about your thoughts, feelings, and behaviors related to your job.

Physical testing will occur at this time, as well as in 6 months, and in 12 months from now. You will be asked to submit the following health measurements: blood pressure, and a measurement of body composition including height and weight. Any problems you have with the health assessment testing will be discussed during this time period, or at any other time you have questions. The surveys and physical testing will take place at your place of work and during your scheduled work hours.

\section{Frequently Asked Questions}

\section{How will my privacy be protected?}

We will protect your privacy in the following ways:

1. Your name or other protected information will not be used in any published documents.

2. Only research personnel associated with the study will be able to access your information and these individuals will keep your information confidential.

3. The code key, linking your name to your participant code and study data will be stored in a locked and password-protected computer file, with access limited only to investigators and data managers.

\section{What are the risks and discomforts?}

There may be personal discomfort when answering questions about personal health behaviors. You may refuse to answer any of the questions. As a result of the measurement of blood pressure, we may find you have a health condition that needs treatment. You would be responsible for the costs of follow-up care and any missed 
workdays. The Principal Investigator can help you find follow-up care if you request his help.

\section{What are the benefits?}

You may or may not personally benefit from being in this study. However, by serving as a subject, you may help us learn how to benefit others in the future.

\section{What are my alternatives?}

You may choose not to be in this study. You can choose not to answer survey items or have measurements made.

\section{Confidentiality and privacy of your protected health information:}

We will not use your name or your identity for publication or publicity purposes. If you sign this form, you are agreeing that PSU may use and publish the health information collected and created in this research study.

You have the right to revoke this authorization and can withdraw your permission for us to use your information for this research by sending a written request to the Investigator listed at the bottom of the research consent form. If you do send a letter to the Principal Investigator, the use and disclosure of your protected health information will stop as of the date she receives your request. However, the Principal Investigator is allowed to use and disclose information collected before the date of the letter or collected in good faith before your letter arrives. The information about you that is used or disclosed in this study may be re-disclosed and no longer protected under federal law.

\section{Costs:}

There will be no direct cost to you for participating in the study.

\section{Liability:}

If you have concerns or questions about your participation in this study or your rights as a research subject, please contact the Human Subjects Research Review Committee, Research + Strategic Partnerships (RSP), PO Box 751, Portland, OR 97207, (503) 725-4288 / 1-877-480-4400. If you have questions about the study itself, please contact Leslie Hammer at (503) 725-3971, hammerl@pdx.edu.

\section{Participation:}

You do not have to join this or any research study. If you do join, and later change your mind, you may quit at any time. If you refuse to join or withdraw early from the study, there will be no penalty or loss of any benefits to which you are otherwise entitled. Your consent to participate in this study and your authorization to let us use and disclose your protected health information are voluntary. You may refuse to sign this consent and authorization form.

You will be given a copy of this consent form for your records. 
Your responses are completely confidential and will not be linked to you in any way. We do not ask you to provide your name or other identifying information on the survey. We ask you to provide the name of your workgroup only so that we can analyze the data by workgroups. The results of this research will only be reported in aggregate form (everyone's information will be pooled together and summarized). No information on any individual or workgroup will be provided to management.

\section{Signatures:}

Your signature below indicates that you have read this entire form and that you agree to be in this study.

\begin{tabular}{lll}
\hline (Participant's name) & (Participant's signature)
\end{tabular}

\begin{tabular}{lll}
\hline $\begin{array}{l}\text { (Name of person obtaining } \\
\text { consent) }\end{array}$ & $\begin{array}{l}\text { (Signature of person obtaining } \\
\text { consent) }\end{array}$ & (Date)
\end{tabular}




\section{Appendix C: Study Questionnaire}

\section{Job Satisfaction}

\begin{tabular}{|c|c|c|c|c|c|c|}
\hline \multirow{4}{*}{$\begin{array}{l}\text { Instructions: Please read each of the } \\
\text { following statements and choose how much } \\
\text { you agree or disagree with each one. }\end{array}$} & & \multicolumn{5}{|c|}{ Strongly Agree } \\
\hline & & \multirow{2}{*}{\multicolumn{5}{|c|}{\begin{tabular}{l}
\multicolumn{2}{c}{ Agree } \\
Neutral
\end{tabular}}} \\
\hline & & & & & & \\
\hline & \multicolumn{6}{|c|}{ Disagree } \\
\hline (fill in one circle for each statement) & Stro & & & & & \\
\hline \multicolumn{2}{|l|}{ 1. In general, you like working at your job. } & 1 & 2 & 3 & 4 & 5 \\
\hline \multicolumn{2}{|l|}{ 2. In general, you are satisfied with your job. } & 1 & 2 & 3 & 4 & 5 \\
\hline \multicolumn{2}{|c|}{$\begin{array}{l}\text { 3. You are generally satisfied with the kind of work you do in this } \\
\text { job. }\end{array}$} & 1 & 2 & & & \\
\hline
\end{tabular}

\section{Job Strain}

\begin{tabular}{|c|c|c|c|c|c|c|}
\hline \multirow{3}{*}{$\begin{array}{l}\text { Instructions: Please show how much you agree or } \\
\text { disagree with each statement by circling one } \\
\text { response next to each statement. }\end{array}$} & & \multicolumn{5}{|c|}{ Strongly Agree } \\
\hline & & \multicolumn{4}{|c|}{ Agree } & \\
\hline & & \multicolumn{4}{|c|}{ Neutral } & \\
\hline \multirow[b]{2}{*}{ (fill in one circle for each statement) } & \multicolumn{4}{|c|}{ Disagree } & & \\
\hline & \multicolumn{2}{|c|}{ Strongly Disagree } & & & & \\
\hline \multicolumn{2}{|l|}{ My job requires that I learn new things. } & 1 & 2 & 3 & 4 & 5 \\
\hline \multicolumn{2}{|c|}{ 2. I have an opportunity to develop my own special abilities. } & 1 & 2 & 3 & 4 & 5 \\
\hline \multicolumn{2}{|l|}{ 3. My job requires a high level of skill. } & 1 & 2 & 3 & 4 & 5 \\
\hline \multicolumn{2}{|l|}{ 4. I get to do a variety of different things on my job. } & 1 & 2 & 3 & 4 & 5 \\
\hline \multicolumn{2}{|l|}{ 5. My job requires a lot of repetitive work. } & 1 & 2 & 3 & 4 & 5 \\
\hline \multicolumn{2}{|l|}{ 6. My job requires me to be creative. } & 1 & 2 & 3 & 4 & 5 \\
\hline \multicolumn{2}{|c|}{ 7. My job allows me to make a lot of decisions on my own. } & 1 & 2 & 3 & 4 & 5 \\
\hline \multicolumn{2}{|c|}{$\begin{array}{l}\text { 8. On my job, I am given a lot of freedom to decide how I do my } \\
\text { work. }\end{array}$} & 1 & 2 & 3 & 4 & 5 \\
\hline \multicolumn{2}{|l|}{ 9. I have a lot of say about what happens on my job. } & 1 & 2 & 3 & 4 & 5 \\
\hline \multicolumn{2}{|l|}{ 10. I am not asked to do an excessive amount of work. } & 1 & 2 & 3 & 4 & 5 \\
\hline \multicolumn{2}{|l|}{ 11. I am free from conflicting demands that others make. } & 1 & 2 & 3 & 4 & 5 \\
\hline \multicolumn{2}{|l|}{ 12. I have enough time to get the job done. } & 1 & 2 & 3 & 4 & \\
\hline \multicolumn{2}{|l|}{ 13. My job requires working very fast. } & 1 & 2 & 3 & 4 & 5 \\
\hline \multicolumn{2}{|l|}{ 14. My job requires working very hard. } & 1 & 2 & & 4 & \\
\hline
\end{tabular}


Organizational Justice - Procedural Justice

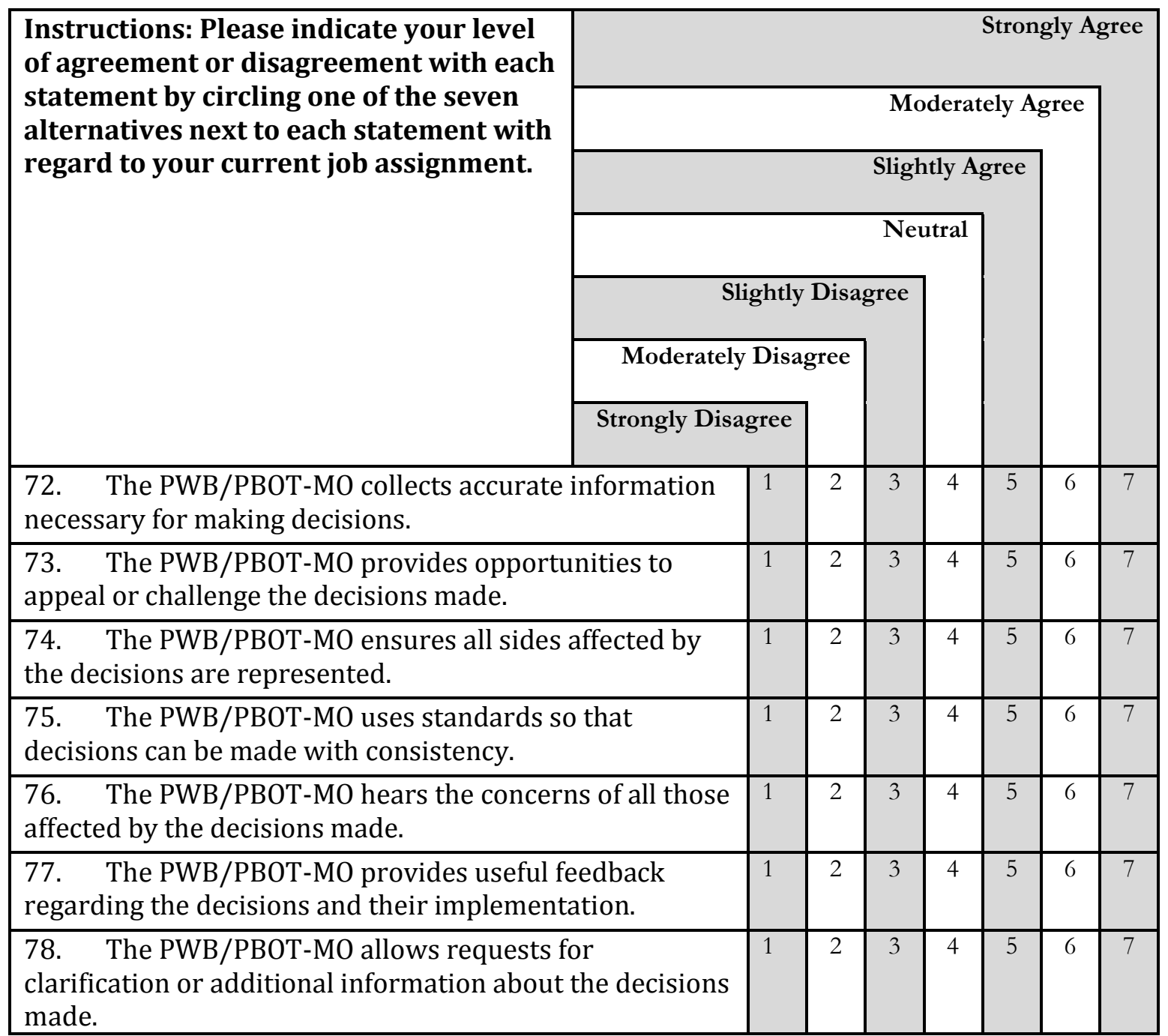


Organizational Justice - Distributive Justice

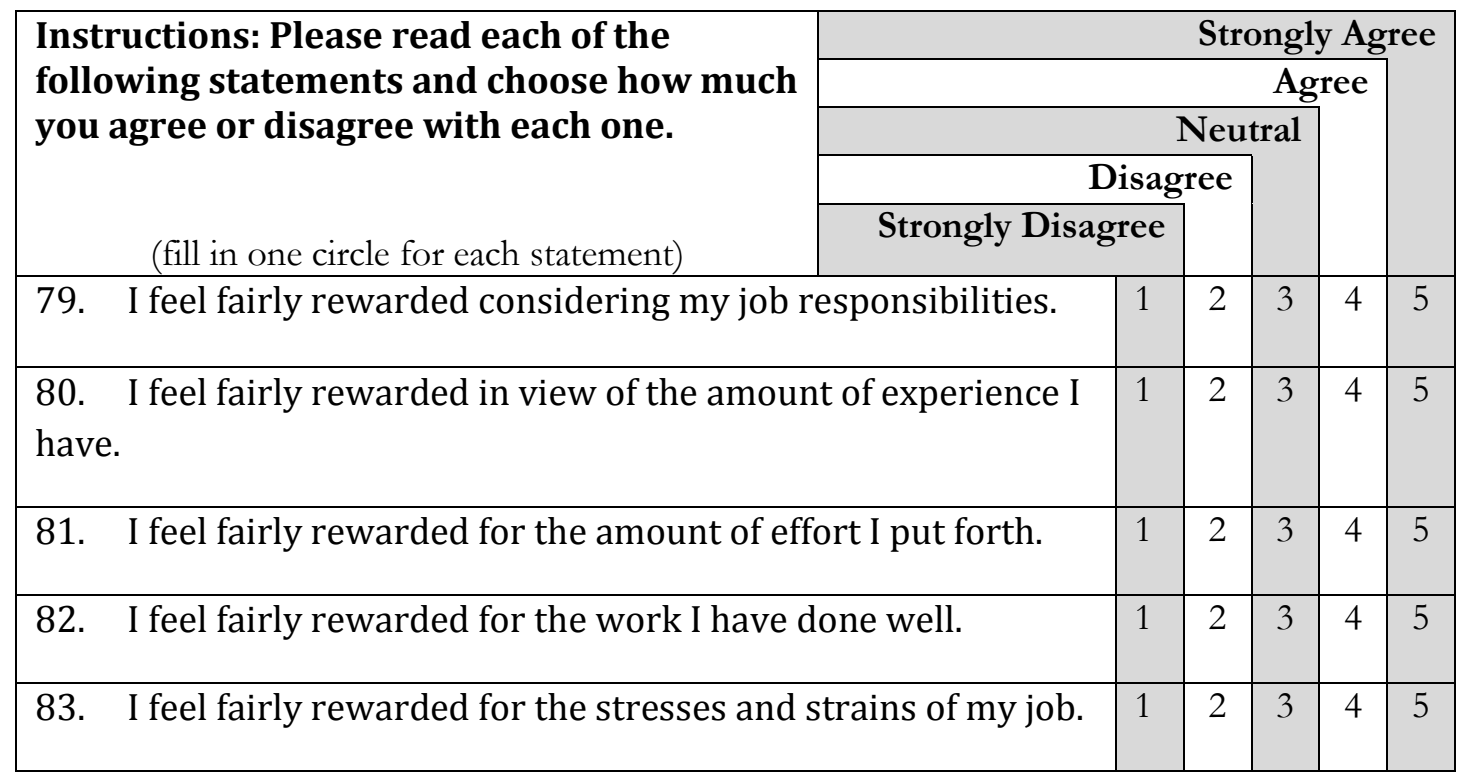

\section{Body Mass Index (BMI)}

Weight and height were captured during the medical assessment and recorded on each participant's Health Assessment Form (Appendix D). The following formula was used to calculate each participant's BMI:

\section{Weight kilograms / Height in meters ${ }^{2}$}

Lean $\mathrm{BMI}<22 \mathrm{~kg} / \mathrm{m}^{2}$

Average BMI $23-27 \mathrm{~kg} / \mathrm{m}^{2}$

High BMI $>27 \mathrm{~kg} / \mathrm{m}^{2}$

Obesity $=\mathrm{BMI}>30 \mathrm{~kg} / \mathrm{m}^{2}$ 


\section{Appendix D: Health Assessment Form}

SHIP Health Assessment I C Participant ID

Date __ Time

$\square$ Station 1: Survey

Tester Initials

Consent? YES

NO_

Survey? YES

NO

Station 2: Blood Pressure/Heart Rate Tester Initials

What is your dominant hand? $\mathrm{R} \quad \mathrm{L}$

Blood pressure medication in last 24 hours? YES__ NO_ Blood Pressure and Heart Rate Readings:

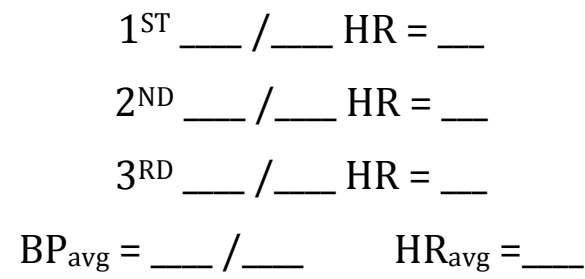

$\square$ Station 3: Anthropometric Measures Tester Initials

Height__ft__ in ___ $\mathrm{cm}$

$\square$ Station 4: Completion

Tester Initials

Completed Health Assessment Form?

YES

$\mathrm{NO}$

Gift Card 